\title{
Cubic color charge correlator in a proton made of three quarks and a gluon
}

\author{
Adrian Dumitru ${ }^{*}$ \\ Department of Natural Sciences, Baruch College, CUNY, 17 Lexington Avenue, \\ New York, New York 10010, USA \\ and The Graduate School and University Center, The City University of New York, 365 Fifth Avenue, \\ New York, New York 10016, USA \\ Heikki Mäntysaariø ${ }^{\dagger}$ \\ Department of Physics, University of Jyväskylä, P.O. Box 35, 40014 University of Jyväskylä, Finland \\ and Helsinki Institute of Physics, P.O. Box 64, 00014 University of Helsinki, Finland
}

Risto Paatelainen $\oplus^{*}$

Helsinki Institute of Physics and Department of Physics, FI-00014 University of Helsinki, Finland

(Received 14 July 2021; accepted 19 January 2022; published 16 February 2022)

\begin{abstract}
The three point correlation function of color charge densities is evaluated explicitly in light -one gauge for a proton on the light cone. This includes both $C$-conjugation even and odd contributions. We account for perturbative corrections to the three-quark light -cone wave function due to the emission of an internal gluon which is not required to be soft. We verify the Ward identity as well as the cancellation of UV divergences in the sum of all diagrams so that the correlator is independent of the renormalization scale. It does, however, exhibit the well-known soft and collinear singularities. The expressions derived here provide the $C$-odd contribution to the initial conditions for high-energy evolution of the dipole scattering amplitude to small $x$. Finally, we also present a numerical model estimate of the impact parameter dependence of quantum color charge three-point correlations in the proton at moderately small $x$.
\end{abstract}

DOI: 10.1103/PhysRevD.105.036007

\section{INTRODUCTION}

In this paper we present explicit expressions for all diagrams which determine the cubic light-cone gauge color charge correlator $\left\langle\rho^{a}\left(\vec{q}_{1}\right) \rho^{b}\left(\vec{q}_{2}\right) \rho^{c}\left(\vec{q}_{3}\right)\right\rangle$ in a proton. The proton is approximated by a nonperturbative three-quark Fock state, plus a perturbative gluon. This is in continuation of Ref. [1] where we derived analogous expressions for $\left\langle\rho^{a}\left(\vec{q}_{1}\right) \rho^{b}\left(\vec{q}_{2}\right)\right\rangle$, and of Ref. [2] where we presented numerical results for the quadratic correlator (see also Ref. [3] for a first phenomenological application). The cubic correlator quantifies corrections to Gaussian color charge fluctuations in the proton, and it provides a contribution to the scattering matrix of a dipole which is odd under $C$-conjugation.

\footnotetext{
*adrian.dumitru@baruch.cuny.edu

†heikki.mantysaari@jyu.fi

*risto.sakari.paatelainen@cern.ch
}

Published by the American Physical Society under the terms of the Creative Commons Attribution 4.0 International license. Further distribution of this work must maintain attribution to the author(s) and the published article's title, journal citation, and DOI. Funded by SCOAP.
The $S$-matrix for eikonal scattering of a quarkantiquark dipole off the fields in the target proton can be expressed as ${ }^{1}[4,5]$

$$
\mathcal{S}(\vec{r}, \vec{b})=\frac{1}{N_{c}} \operatorname{tr}\left\langle U\left(\vec{b}+\frac{\vec{r}}{2}\right) U^{\dagger}\left(\vec{b}-\frac{\vec{r}}{2}\right)\right\rangle .
$$

Here, $\vec{b}$ is the impact parameter vector while $\vec{r}$ denotes the transverse separation of quark and antiquark. The operators $U\left(U^{\dagger}\right)$ are (anti)path ordered Wilson lines of the field in covariant gauge, representing the eikonal scattering of the quarks at transverse coordinate $\vec{x}$ :

$U(\vec{x})=\mathcal{P} e^{i g \int \mathrm{d} x^{-} A^{+a}\left(x^{-}, \vec{x}\right) t^{a}}, \quad U^{\dagger}(\vec{x})=\overline{\mathcal{P}} e^{-i g \int \mathrm{d} x^{-} A^{+a}\left(x^{-}, \vec{x}\right) t^{a}}$.

$C$-conjugation transforms the generators of the fundamental representation $t^{a} \rightarrow-\left(t^{a}\right)^{T}$.

The $S$-matrix can be separated into its real part which at high energy is dominated by $C$-conjugation even two-gluon

\footnotetext{
${ }^{1}\langle\cdots\rangle$ denotes the matrix element between incoming and outgoing proton states. It is defined in Eq. (9) below.
} 
exchange, and its imaginary part which starts out as $C$-odd three gluon exchange:

$$
\begin{aligned}
D(\vec{r}, \vec{b}) \equiv & \operatorname{Re} \mathcal{S}(\vec{r}, \vec{b})=\frac{1}{2 N_{c}} \operatorname{tr}\left\langle U\left(\vec{b}+\frac{\vec{r}}{2}\right) U^{\dagger}\left(\vec{b}-\frac{\vec{r}}{2}\right)\right. \\
& \left.+U\left(\vec{b}-\frac{\vec{r}}{2}\right) U^{\dagger}\left(\vec{b}+\frac{\vec{r}}{2}\right)\right\rangle, \\
O(\vec{r}, \vec{b}) \equiv & \operatorname{Im} \mathcal{S}(\vec{r}, \vec{b})=\frac{1}{2 i N_{c}} \operatorname{tr}\left\langle U\left(\vec{b}+\frac{\vec{r}}{2}\right) U^{\dagger}\left(\vec{b}-\frac{\vec{r}}{2}\right)\right. \\
& \left.-U\left(\vec{b}-\frac{\vec{r}}{2}\right) U^{\dagger}\left(\vec{b}+\frac{\vec{r}}{2}\right)\right\rangle .
\end{aligned}
$$

Thus, the fact that the imaginary part of $\mathcal{S}(\vec{r}, \vec{b})$ is nonzero is due to the existence of a color singlet three gluon ( $t$-channel) exchange with negative $C$-parity in QCD [6-11]. Recently, the TOTEM and D0 Collaborations have presented evidence for a difference in $p-p$ vs $p-\bar{p}$ elastic scattering cross sections at a $\mathrm{CM}$ energy of $\sqrt{s} \simeq 2 \mathrm{TeV}$, and low momentum transfer $|t|<1 \mathrm{GeV}^{2}$ $[12,13]$ (also see Ref. [14]). However, our focus here is on cubic color charge correlations in the semihard regime, which is related to the $C$ and $P$ odd contribution to the dipole scattering amplitude.

The evolution of the dipole $S$-matrix in the high-energy regime is described by the Jalilian-Marian, Iancu, McLerran, Weigert, Leonidov and Kovner (JIMWLK) renormalization group equations [15-24]. These reduce in the large- $N_{c}$ limit to the Balitsky-Kovchegov (BK) equation $[15,25]$. High-energy resummation may modify the intercept of the "hard Odderon" from its value of unity; see the review [10] and references therein. The evolution with energy specifically of the (hard) Odderon $O(\vec{r}, \vec{b})$ has been studied in Refs. [26-28]. Its knowledge is important for various spin dependent transverse momentum dependent (TMD) distributions such as the (dipole) gluon Sivers function of a transversely polarized proton [29-31]. Furthermore, this amplitude is responsible for charge asymmetries in diffractive electroproduction of a $\pi^{+} \pi^{-}$ pair [32,33], and exclusive production of a pseudoscalar meson [34-38]. Last, the odderon is related to cubic color charge density fluctuations $\left\langle\rho^{a}\left(\vec{q}_{1}\right) \rho^{b}\left(\vec{q}_{2}\right) \rho^{c}\left(\vec{q}_{3}\right)\right\rangle$ (see below) and therefore provides insight into three-body correlations in the proton. This may guide phenomenological models of correlated "hot spots" which have been applied to proton-proton scattering at high energies [39-42] (see also Ref. [43]). The existence of the cubic correlator also implies that color charge density fluctuations in the proton are not Gaussian.

A key limitation for quantitative predictions in the energy regime of the Electron-Ion Collider (EIC) [44-47] is the crude knowledge of the initial condition for the evolution equations at moderately small $x$. Deriving the next-to-leading order (NLO) expressions for $O(\vec{r}, \vec{b})$ due to one gluon emission corrections in a proton target at $x \sim$ $0.01-0.1$ is the main purpose of this paper. The corresponding expressions at leading order (LO) have been published in Refs. [34,48,49]. The latter paper also provides numerical estimates of cubic color charge correlators and of $O(\vec{r}, \vec{b})$ at LO, i.e., in the valence quark regime. Bartels and Motyka [50] have also calculated the proton impact factor for $t$-channel three gluon exchange, which agrees with the LO expressions for $\left\langle\rho^{a}\left(\vec{q}_{1}\right) \rho^{b}\left(\vec{q}_{2}\right) \rho^{c}\left(\vec{q}_{3}\right)\right\rangle$ given in Refs. [34,48,49], and soft gluon emission corrections to proton-proton scattering at high energy.

The initial condition for the small- $x$ evolution [26-28] of $\operatorname{Im} \mathcal{S}$, which we derive here, depends not only on the impact parameter and the dipole vectors but also on their relative angle, and on the light-cone momentum fraction $x$ in the target. In fact, the BK equation in its standard formulation evolves the wave function of the dipole projectile, and the evolution "time" is then related to the minus component of the momentum of the gluon in the proton target [51-53]. Ducloue et al. have reformulated [52] BK evolution at NLO in terms of the target rapidity (or Bjorken- $x$ ). They obtained an evolution equation which is nonlocal in rapidity and which depends explicitly on the gluon's plus momentum fraction $x=k_{g}^{+} / P^{+}$. Therefore, it is important to determine the dependence of the initial scattering amplitude not only on impact parameter $\vec{b}$ and dipole size $\vec{r}$ but also its dependence on $x$.

The amplitude for $C$-odd three gluon exchange is related to the correlator of $C$-odd color charge fluctuations $[34,48,49],{ }^{2}$

$$
\begin{aligned}
-i O(\vec{r}, \vec{b})= & -\frac{5}{18} g^{6} \int_{q_{1}, q_{2}, q_{3}} \frac{1}{q_{1}^{2}} \frac{1}{q_{2}^{2}} \frac{1}{q_{3}^{2}} e^{-i \vec{b} \cdot \vec{K}} G_{3}^{-}\left(\vec{q}_{1}, \vec{q}_{2}, \vec{q}_{3}\right) \\
& \times\left[\sin \left(\vec{r} \cdot \vec{q}_{1}+\frac{1}{2} \vec{r} \cdot \vec{K}\right)-\frac{1}{3} \sin \left(\frac{1}{2} \vec{r} \cdot \vec{K}\right)\right] .
\end{aligned}
$$

Here, $\vec{K}=-\left(\vec{q}_{1}+\vec{q}_{2}+\vec{q}_{3}\right)$ is the (transverse) momentum transfer given $\vec{P}=0$ for the incoming proton, and $\int_{q}$ is shorthand for $\int \mathrm{d}^{2} q /(2 \pi)^{2}$. We denote the $C$-odd part of the correlator of three color charges as

$\left\langle\rho^{a}\left(\vec{q}_{1}\right) \rho^{b}\left(\vec{q}_{2}\right) \rho^{c}\left(\vec{q}_{3}\right)\right\rangle_{C=-} \equiv \frac{1}{4} d^{a b c} g^{3} G_{3}^{-}\left(\vec{q}_{1}, \vec{q}_{2}, \vec{q}_{3}\right)$.

Note that $G_{3}^{-}\left(\vec{q}_{1}, \vec{q}_{2}, \vec{q}_{3}\right)$ from Eq. (5) is given by the correlator of three covariant-gauge color charge densities.

\footnotetext{
${ }^{2}$ The sign of $\mathcal{T}_{\text {ggg }}(\vec{r}, \vec{b})$ in Eq. (5) differs from Ref. [34] because here we follow the convention of Kovchegov and Sievert [54] with $+i g$ in the exponent of the Wilson line $U(\vec{x})$ in Eq. (2), and with the covariant gauge operator relation $-\nabla_{\perp}^{2} \int \mathrm{d} x^{-} A^{+a}\left(x^{-}, \vec{x}\right)=\rho^{a}(\vec{x})$.
} 
However, in the weak field limit, a computation in light-cone gauge is applicable.

The above correlator is symmetric under a simultaneous sign flip of all three gluon momenta, and so $-i \operatorname{Im} \mathcal{S}(\vec{r}, \vec{b})=$ $-i O(\vec{r}, \vec{b})$ is imaginary. ${ }^{3}$ Also, it vanishes quadratically in any of the transverse momentum arguments so that $-i O(\vec{r}, \vec{b})$ is free of infrared divergences. The light-cone gauge color charge density operator in the eikonal "shock wave limit" is given by $\rho^{a}(\vec{k})=\rho_{\mathrm{qu}}^{a}(\vec{k})+\rho_{\mathrm{gl}}^{a}(\vec{k})$ with [1]

$\rho_{\mathrm{qu}}^{a}(\vec{k})=g \sum_{i, j, \sigma}\left(t^{a}\right)_{i j} \int \frac{\mathrm{d} x_{q} \mathrm{~d}^{2} q}{16 \pi^{3} x_{q}} b_{i \sigma}^{\dagger}\left(x_{q}, \vec{q}\right) b_{j \sigma}\left(x_{q}, \vec{k}+\vec{q}\right)$,

$\rho_{\mathrm{gl}}^{a}(\vec{k})=g \sum_{\lambda b c}\left(T^{a}\right)_{b c} \int \frac{\mathrm{d} x_{g} \mathrm{~d}^{2} q}{16 \pi^{3} x_{g}} a_{b \lambda}^{\dagger}\left(x_{g}, \vec{q}\right) a_{c \lambda}\left(x_{g}, \vec{q}+\vec{k}\right)$.

Here $a^{\dagger}, a$ and $b^{\dagger}, b$ denote creation and annihilation operators for gluons and quarks, respectively.

In Sec. II we compute all contributions to $\left\langle\rho^{a}\left(\vec{q}_{1}\right) \rho^{b}\left(\vec{q}_{2}\right) \rho^{c}\left(\vec{q}_{3}\right)\right\rangle$ in a proton on the light cone in light-cone gauge; specifically we consider the NLO correction due to the emission or exchange of a gluon which is not required to be soft. In Sec. III we describe the Fourier transform of the correlator to impact parameter space, and present a numerical model estimate. A brief summary is presented in Sec. IV. Appendix A summarizes the Fock state description of the proton on the light front used throughout this paper, Appendix B shows the cancellation of UV divergences in the sum of all diagrams for $\left\langle\rho^{a}\left(\vec{q}_{1}\right) \rho^{b}\left(\vec{q}_{2}\right) \rho^{c}\left(\vec{q}_{3}\right)\right\rangle$, and in Appendix $\mathrm{C}$ we check the vanishing of this correlator when $\vec{q}_{1} \rightarrow 0$ or $\vec{q}_{3} \rightarrow 0$.

\section{CORRELATOR OF THREE COLOR CHARGE OPERATORS, $\left\langle\boldsymbol{\rho}^{a}\left(\vec{q}_{1}\right) \boldsymbol{\rho}^{b}\left(\vec{q}_{2}\right) \rho^{c}\left(\vec{q}_{3}\right)\right\rangle$}

In this section we compute the correlator of three color charge operators $\left\langle\rho^{a}\left(\vec{q}_{1}\right) \rho^{b}\left(\vec{q}_{2}\right) \rho^{c}\left(\vec{q}_{3}\right)\right\rangle$ where $\rho^{a}(\vec{q})=\rho_{\mathrm{gl}}^{a}(\vec{q})+\rho_{\mathrm{qu}}^{a}(\vec{q})$. This expectation value is defined as the matrix element of the product of three color charge operators between the incoming $(|P\rangle)$ and outgoing $(\langle K|)$ proton states, stripped of the delta functions expressing conservation of plus and transverse momenta:

$$
\begin{aligned}
& 16 \pi^{3} P^{+} \delta\left(P^{+}-K^{+}\right) \delta\left(\vec{P}-\vec{K}-\sum_{i} \vec{q}_{i}\right) \\
& \quad \times\left\langle\rho^{a}\left(\vec{q}_{1}\right) \rho^{b}\left(\vec{q}_{2}\right) \rho^{c}\left(\vec{q}_{3}\right)\right\rangle \equiv\left\langle K\left|\rho^{a}\left(\vec{q}_{1}\right) \rho^{b}\left(\vec{q}_{2}\right) \rho^{c}\left(\vec{q}_{3}\right)\right| P\right\rangle .
\end{aligned}
$$

The structure of the proton state assumed in this work is explained briefly in Appendix A.

\footnotetext{
${ }^{3}$ In mixed representation $i O(\vec{r}, \vec{K})$ is real, however.
}

In general, this correlator has both even and odd components under $C$-parity which transforms $\left(t^{a}\right)_{i j} \rightarrow-\left(t^{a}\right)_{j i}{ }^{4}$ and $\left(T^{a}\right)_{b c} \rightarrow-\left(T^{a}\right)_{c b}=\left(T^{a}\right)_{b c}{ }^{5}$ Note that the following expressions apply when the number of colors $N_{c}=3$.

We shall use the shorthand notation $\vec{q}=\vec{q}_{1}+\vec{q}_{2}+\vec{q}_{3}=$ $\vec{P}-\vec{K}$ and $\vec{q}_{i j}=\vec{q}_{i}+\vec{q}_{j}$ in the following expressions. ${ }^{6}$ Their corresponding diagrams are shown in the figures. We label them as Fig. 1 $\left(q_{3} q_{2} g\right)$, for example, corresponding to a diagram of the type shown in Fig. 1 (i.e., a gluon exchange across the operator insertion by a quark with itself, with at least one of the probes attached to that internal gluon) where the first probe gluon (momentum $\vec{q}_{1}$, color $a$ ) couples to the internal gluon, the second probe gluon (momentum $\vec{q}_{2}$, color $b$ ) couples to the second quark, and the third probe (momentum $\vec{q}_{3}$, color $c$ ) couples to the third quark.

\section{A. UV divergent diagrams}

We begin with the UV divergent diagrams where a quark exchanges a gluon with itself. The diagrams where one or more of the probes attach to the gluon are shown in Fig. 1.

To prepare, we first list the matrix elements of one, two, and three $\rho_{\mathrm{gl}}(\vec{q})$ between one-gluon states:

$$
\begin{aligned}
\left\langle\ell, \rho, d\left|\rho_{\mathrm{gl}}^{a}\left(\vec{q}_{1}\right)\right| k_{g}, \sigma, c\right\rangle= & g\left(T^{a}\right)_{d c} \delta_{\rho \sigma}(2 \pi)^{D-1} 2 k_{g}^{+} \\
& \times \delta\left(k_{g}^{+}-\ell^{+}\right) \delta\left(\vec{k}_{g}-\vec{\ell}-\vec{q}_{1}\right),
\end{aligned}
$$

$$
\begin{aligned}
\left\langle\ell, \rho, d\left|\rho_{\mathrm{gl}}^{a}\left(\vec{q}_{1}\right) \rho_{\mathrm{gl}}^{b}\left(\vec{q}_{2}\right)\right| k_{g}, \sigma, c\right\rangle= & g^{2}\left(T^{a} T^{b}\right)_{d c} \delta_{\rho \sigma}(2 \pi)^{D-1} \\
& \times 2 k_{g}^{+} \delta\left(k_{g}^{+}-\ell^{+}\right) \\
& \times \delta\left(\vec{k}_{g}-\vec{\ell}-\vec{q}_{1}-\vec{q}_{2}\right),
\end{aligned}
$$

$$
\begin{aligned}
& \left\langle\ell, \rho, d\left|\rho_{\mathrm{gl}}^{a}\left(\vec{q}_{1}\right) \rho_{\mathrm{gl}}^{b}\left(\vec{q}_{2}\right) \rho_{\mathrm{gl}}^{c}\left(\vec{q}_{3}\right)\right| k_{g}, \sigma, e\right\rangle \\
& =g^{3}\left(T^{a} T^{b} T^{c}\right)_{d e} \delta_{\rho \sigma}(2 \pi)^{D-1} 2 k_{g}^{+} \delta\left(k_{g}^{+}-\ell^{+}\right) \\
& \quad \times \delta\left(\vec{k}_{g}-\vec{\ell}-\vec{q}_{1}-\vec{q}_{2}-\vec{q}_{3}\right) .
\end{aligned}
$$

The matrix elements of $\rho_{\mathrm{qu}}(\vec{q})$ between one-quark states are similar, with $T^{a} \rightarrow t^{a}$.

${ }^{4}$ Hence, $\operatorname{tr} t^{a} t^{b} t^{c}=\frac{1}{4}\left(d^{a b c}+i f^{a b c}\right) \rightarrow-\operatorname{tr} t^{c} t^{b} t^{a}=-\frac{1}{4}\left(d^{a b c}-i f^{a b c}\right)$. Therefore, terms proportional to $d^{a b c}$ are odd under $C$-conjugation while terms proportional to if ${ }^{a b c}$ are even.

${ }^{5}$ One may also classify according to "signature," i.e., the sign under exchange of the colors and momenta of any two external gluons (charge operators).

${ }^{6}$ Thus, the notation here is different from Refs. [1,2] where $\vec{q}_{12}$ stood for $\vec{q}_{1}-\vec{q}_{2}$. 


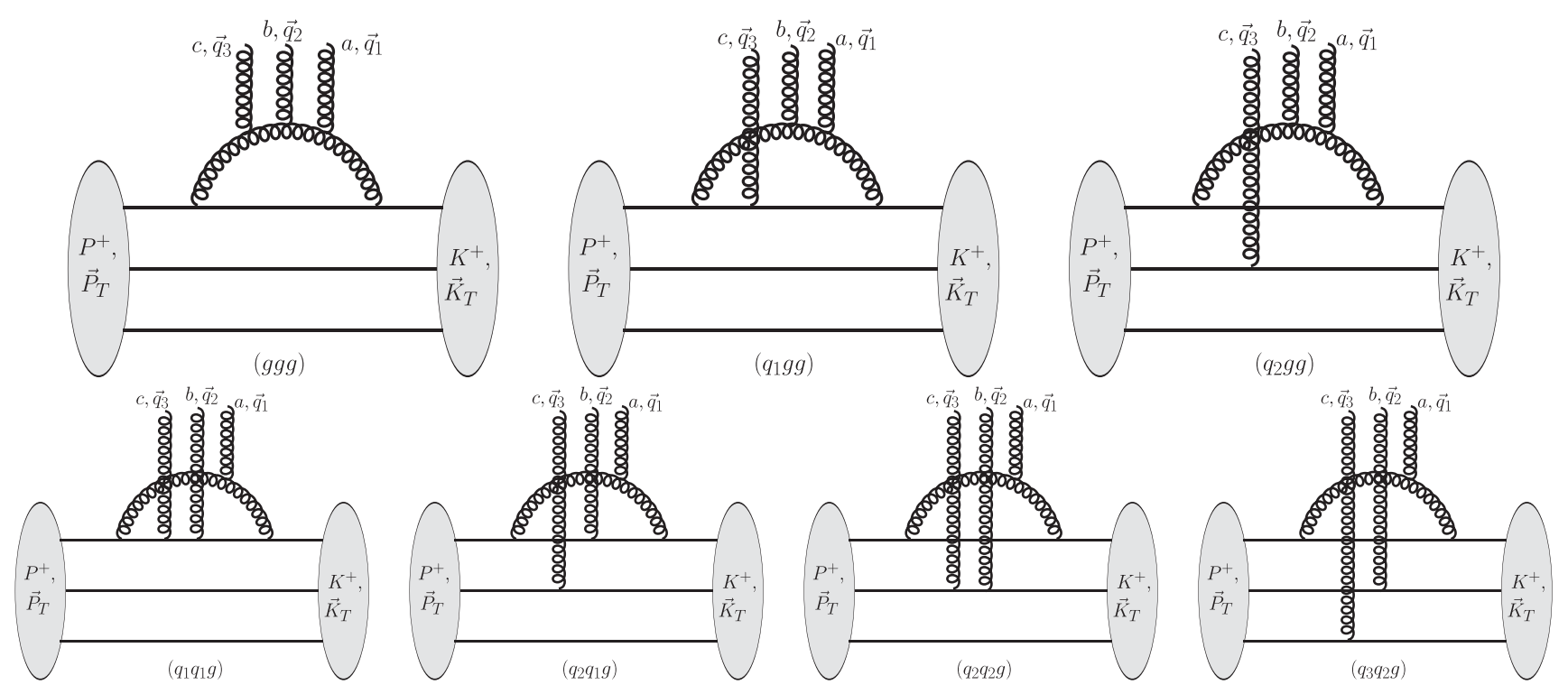

FIG. 1. UV divergent diagrams (propagators of external probes to be amputated) for $\left\langle\rho^{a}\left(\vec{q}_{1}\right) \rho^{b}\left(\vec{q}_{2}\right) \rho^{c}\left(\vec{q}_{3}\right)\right\rangle$ where at least one of the probes attaches to the gluon in the proton. The cut is located at the insertion of the three color charge operators.

With this we obtain

$$
\begin{aligned}
& \text { Fig. } 1(g g g)=\frac{2 g^{5}}{3 \cdot 16 \pi^{3}} \operatorname{tr} T^{a} T^{b} T^{c} \int\left[\mathrm{d} x_{i}\right] \int\left[\mathrm{d}^{2} k_{i}\right] \Psi_{q q q}\left(x_{1}, \vec{k}_{1} ; x_{2}, \vec{k}_{2} ; x_{3}, \vec{k}_{3}\right) \\
& \times \Psi_{q q q}^{*}\left(x_{1}, \vec{k}_{1}-\left(1-x_{1}\right) \vec{q} ; x_{2}, \vec{k}_{2}+x_{2} \vec{q} ; x_{3}, \vec{k}_{3}+x_{3} \vec{q}\right) \\
& \times \frac{(2 \pi)^{D-1}}{2 p_{1}^{+}} \int \frac{\widetilde{\mathrm{d}}_{g}}{2\left(p_{1}^{+}-k_{g}^{+}\right)}\left\langle S\left|\hat{\psi}_{q \rightarrow q g}\left(\vec{p}_{1} ; \vec{p}_{1}-\vec{k}_{g}, \vec{k}_{g}\right) \hat{\psi}_{q \rightarrow q g}^{*}\left(\vec{p}_{1}-\vec{q} ; \vec{p}_{1}-\vec{k}_{g}, \vec{k}_{g}-\vec{q}\right)\right| S\right\rangle,
\end{aligned}
$$

with a symmetry factor of 3 . Here the integration measures $\left[\mathrm{d} k_{i}\right]$ and $\left[\mathrm{d} x_{i}\right]$ and the proton valence quark wave function $\Psi_{q q q}$ are defined in Appendix A 1. The Lorentz invariant gluon phase space measure $\widetilde{\mathrm{d}}_{g}$ is given in Appendix A 2, the phase space integral is calculated in Ref. [1], and the result is also included in Appendix B. Since $\operatorname{tr} T^{a} T^{b} T^{c}=\frac{i}{2} N_{c} f^{a b c}=-\frac{3}{2}\left(T^{a}\right)_{b c}$, it follows that this contribution is even under $C$ conjugation. The remaining integral over the longitudinal and transverse momenta of the emitted gluon can be decomposed into a finite function and a UV divergent part [1]. We verify the cancellation of the UV divergences in Appendix B,

$$
\begin{aligned}
& \text { Fig. } 1\left(q_{1} g g\right)=\frac{2 g^{5}}{3 \cdot 16 \pi^{3}} \frac{1}{2} \operatorname{tr}\left(T^{a} T^{b} D^{c}-T^{a} T^{b} T^{c}\right) \int\left[\mathrm{d} x_{i}\right] \int\left[\mathrm{d}^{2} k_{i}\right] \Psi_{q q q}\left(x_{1}, \vec{k}_{1} ; x_{2}, \vec{k}_{2} ; x_{3}, \vec{k}_{3}\right) \\
& \times \Psi_{q q q}^{*}\left(x_{1}, \vec{k}_{1}-\left(1-x_{1}\right) \vec{q} ; x_{2}, \vec{k}_{2}+x_{2} \vec{q} ; x_{3}, \vec{k}_{3}+x_{3} \vec{q}\right) \\
& \times \frac{(2 \pi)^{D-1}}{2 p_{1}^{+}} \int \frac{\widetilde{\mathrm{d}}_{g}}{2\left(p_{1}^{+}-k_{g}^{+}\right)}\left\langle S\left|\hat{\psi}_{q \rightarrow q g}\left(\vec{p}_{1} ; \vec{p}_{1}-\vec{k}_{g}, \vec{k}_{g}\right) \hat{\psi}_{q \rightarrow q g}^{*}\left(\vec{p}_{1}-\vec{q} ; \vec{p}_{1}-\vec{k}_{g}-\vec{q}_{3}, \vec{k}_{g}-\vec{q}_{12}\right)\right| S\right\rangle,
\end{aligned}
$$

where $\left(D^{c}\right)_{a b}=d^{c a b}$. Performing the traces, the color factor becomes $\frac{1}{2} \operatorname{tr}\left(D^{c} T^{a} T^{b}-\operatorname{tr} T^{b} T^{c} T^{a}\right)=\frac{N_{c}}{4}\left(d^{a b c}-i f^{a b c}\right)$. The first term corresponds to a $C$-odd contribution while the second one is even under $C$. The symmetry factor for this diagram is 3 . 
The diagram where instead the second gluon attaches to quark 1 (not shown) is given by

$$
\begin{aligned}
& \text { Fig. } 1\left(g q_{1} g\right)=\frac{2 g^{5}}{3 \cdot 16 \pi^{3}} \frac{1}{2} \operatorname{tr}\left(T^{a} T^{c} D^{b}-T^{a} T^{c} T^{b}\right) \int\left[\mathrm{d} x_{i}\right] \int\left[\mathrm{d}^{2} k_{i}\right] \Psi_{q q q}\left(x_{1}, \vec{k}_{1} ; x_{2}, \vec{k}_{2} ; x_{3}, \vec{k}_{3}\right) \\
& \times \Psi_{q q q}^{*}\left(x_{1}, \vec{k}_{1}-\left(1-x_{1}\right) \vec{q} ; x_{2}, \vec{k}_{2}+x_{2} \vec{q} ; x_{3}, \vec{k}_{3}+x_{3} \vec{q}\right) \\
& \times \frac{(2 \pi)^{D-1}}{2 p_{1}^{+}} \int \frac{\widetilde{\mathrm{d}}_{g}}{2\left(p_{1}^{+}-k_{g}^{+}\right)}\left\langle S\left|\hat{\psi}_{q \rightarrow q g}\left(\vec{p}_{1} ; \vec{p}_{1}-\vec{k}_{g}, \vec{k}_{g}\right) \hat{\psi}_{q \rightarrow q g}^{*}\left(\vec{p}_{1}-\vec{q} ; \vec{p}_{1}-\vec{k}_{g}-\vec{q}_{2}, \vec{k}_{g}-\vec{q}_{13}\right)\right| S\right\rangle,
\end{aligned}
$$

and the one where the first gluon attaches to quark 1:

$$
\begin{aligned}
& \text { Fig. } 1\left(g g q_{1}\right)=\frac{2 g^{5}}{3 \cdot 16 \pi^{3}} \frac{1}{2} \operatorname{tr}\left(T^{b} T^{c} D^{a}-T^{a} T^{b} T^{c}\right) \int\left[\mathrm{d} x_{i}\right] \int\left[\mathrm{d}^{2} k_{i}\right] \Psi_{q q q}\left(x_{1}, \vec{k}_{1} ; x_{2}, \vec{k}_{2} ; x_{3}, \vec{k}_{3}\right) \\
& \times \Psi_{q q q}^{*}\left(x_{1}, \vec{k}_{1}-\left(1-x_{1}\right) \vec{q} ; x_{2}, \vec{k}_{2}+x_{2} \vec{q} ; x_{3}, \vec{k}_{3}+x_{3} \vec{q}\right) \\
& \times \frac{(2 \pi)^{D-1}}{2 p_{1}^{+}} \int \frac{\widetilde{\mathrm{d}} k_{g}}{2\left(p_{1}^{+}-k_{g}^{+}\right)}\left\langle S\left|\hat{\psi}_{q \rightarrow q g}\left(\vec{p}_{1} ; \vec{p}_{1}-\vec{k}_{g}, \vec{k}_{g}\right) \hat{\psi}_{q \rightarrow q g}^{*}\left(\vec{p}_{1}-\vec{q} ; \vec{p}_{1}-\vec{k}_{g}-\vec{q}_{1}, \vec{k}_{g}-\vec{q}_{23}\right)\right| S\right\rangle .
\end{aligned}
$$

These diagrams come with a symmetry factor of 3 since the "active" quark may just as well be quark 2 or quark 3 .

Continuing with the diagram where the third probe attaches to quark 2 ,

$$
\begin{aligned}
\text { Fig. } 1\left(q_{2} g g\right)= & -\frac{2 g^{5}}{3 \cdot 16 \pi^{3}} \frac{1}{4} \operatorname{tr}\left(T^{a} T^{b} D^{c}+T^{a} T^{b} T^{c}\right) \int\left[\mathrm{d} x_{i}\right] \int\left[\mathrm{d}^{2} k_{i}\right] \Psi_{q q q}\left(x_{1}, \vec{k}_{1} ; x_{2}, \vec{k}_{2} ; x_{3}, \vec{k}_{3}\right) \\
& \times \Psi_{q q q}^{*}\left(x_{1}, \vec{k}_{1}+x_{1} \vec{q}-\vec{q}_{12} ; x_{2}, \vec{k}_{2}+x_{2} \vec{q}-\vec{q}_{3} ; x_{3}, \vec{k}_{3}+x_{3} \vec{q}\right) \\
& \times \frac{(2 \pi)^{D-1}}{2 p_{1}^{+}} \int \frac{\widetilde{\mathrm{d}}_{g}}{2\left(p_{1}^{+}-k_{g}^{+}\right)}\left\langle S\left|\hat{\psi}_{q \rightarrow q g}\left(\vec{p}_{1} ; \vec{p}_{1}-\vec{k}_{g}, \vec{k}_{g}\right) \hat{\psi}_{q \rightarrow q g}^{*}\left(\vec{p}_{1}-\vec{q}_{12} ; \vec{p}_{1}-\vec{k}_{g}, \vec{k}_{g}-\vec{q}_{12}\right)\right| S\right\rangle
\end{aligned}
$$

The symmetry factor is 6 because the third gluon probe may also attach to quark 3 .

Once again there are analogous diagrams (not shown) where the second or the first probe attaches to quark 2 (or to quark 3):

$$
\begin{aligned}
\text { Fig. } 1\left(g q_{2} g\right)= & -\frac{2 g^{5}}{3 \cdot 16 \pi^{3}} \frac{1}{4} \operatorname{tr}\left(T^{a} T^{c} D^{b}+T^{a} T^{c} T^{b}\right) \int\left[\mathrm{d} x_{i}\right] \int\left[\mathrm{d}^{2} k_{i}\right] \Psi_{q q q}\left(x_{1}, \vec{k}_{1} ; x_{2}, \vec{k}_{2} ; x_{3}, \vec{k}_{3}\right) \\
& \times \Psi_{q q q}^{*}\left(x_{1}, \vec{k}_{1}+x_{1} \vec{q}-\vec{q}_{13} ; x_{2}, \vec{k}_{2}+x_{2} \vec{q}-\vec{q}_{2} ; x_{3}, \vec{k}_{3}+x_{3} \vec{q}\right) \\
& \times \frac{(2 \pi)^{D-1}}{2 p_{1}^{+}} \int \frac{\widetilde{\mathrm{d}}_{g}}{2\left(p_{1}^{+}-k_{g}^{+}\right)}\left\langle S\left|\hat{\psi}_{q \rightarrow q g}\left(\vec{p}_{1} ; \vec{p}_{1}-\vec{k}_{g}, \vec{k}_{g}\right) \hat{\psi}_{q \rightarrow q g}^{*}\left(\vec{p}_{1}-\vec{q}_{13} ; \vec{p}_{1}-\vec{k}_{g}, \vec{k}_{g}-\vec{q}_{13}\right)\right| S\right\rangle,
\end{aligned}
$$

$$
\begin{aligned}
\text { Fig.1 }\left(g g q_{2}\right)= & -\frac{2 g^{5}}{3 \cdot 16 \pi^{3}} \frac{1}{4} \operatorname{tr}\left(T^{b} T^{c} D^{a}+T^{a} T^{b} T^{c}\right) \int\left[\mathrm{d} x_{i}\right] \int\left[\mathrm{d}^{2} k_{i}\right] \Psi_{q q q}\left(x_{1}, \vec{k}_{1} ; x_{2}, \vec{k}_{2} ; x_{3}, \vec{k}_{3}\right) \\
& \times \Psi_{q q q}^{*}\left(x_{1}, \vec{k}_{1}+x_{1} \vec{q}-\vec{q}_{23} ; x_{2}, \vec{k}_{2}+x_{2} \vec{q}-\vec{q}_{1} ; x_{3}, \vec{k}_{3}+x_{3} \vec{q}\right) \\
& \times \frac{(2 \pi)^{D-1}}{2 p_{1}^{+}} \int \frac{\widetilde{\mathrm{d}}_{g}}{2\left(p_{1}^{+}-k_{g}^{+}\right)}\left\langle S\left|\hat{\psi}_{q \rightarrow q g}\left(\vec{p}_{1} ; \vec{p}_{1}-\vec{k}_{g}, \vec{k}_{g}\right) \hat{\psi}_{q \rightarrow q g}^{*}\left(\vec{p}_{1}-\vec{q}_{23} ; \vec{p}_{1}-\vec{k}_{g}, \vec{k}_{g}-\vec{q}_{23}\right)\right| S\right\rangle .
\end{aligned}
$$

Their symmetry factors are 6 . 
We continue with the diagrams where two of the probes attach to quarks,

$$
\begin{aligned}
& \text { Fig. } 1\left(q_{1} q_{1} g\right)=-\frac{2 g^{5}}{3 \cdot 16 \pi^{3}} N_{c} \operatorname{tr} t^{a} t^{b} t^{c} \int\left[\mathrm{d} x_{i}\right] \int\left[\mathrm{d}^{2} k_{i}\right] \Psi_{q q q}\left(x_{1}, \vec{k}_{1} ; x_{2}, \vec{k}_{2} ; x_{3}, \vec{k}_{3}\right) \\
& \times \Psi_{q q q}^{*}\left(x_{1}, \vec{k}_{1}+x_{1} \vec{q}-\vec{q} ; x_{2}, \vec{k}_{2}+x_{2} \vec{q} ; x_{3}, \vec{k}_{3}+x_{3} \vec{q}\right) \\
& \times \frac{(2 \pi)^{D-1}}{2 p_{1}^{+}} \int \frac{\widetilde{\mathrm{d}}_{g}}{2\left(p_{1}^{+}-k_{g}^{+}\right)}\left\langle S\left|\hat{\psi}_{q \rightarrow q g}\left(\vec{p}_{1} ; \vec{p}_{1}-\vec{k}_{g}, \vec{k}_{g}\right) \hat{\psi}_{q \rightarrow q g}^{*}\left(\vec{p}_{1}-\vec{q} ; \vec{p}_{1}-\vec{k}_{g}-\vec{q}_{23}, \vec{k}_{g}-\vec{q}_{1}\right)\right| S\right\rangle .
\end{aligned}
$$

The symmetry factor is 3. The $\mathrm{SU}\left(N_{c}\right)$ relation $f^{a b c} t^{a} t^{b}=\frac{i}{2} N_{c} t^{c}$ is useful for evaluating the color factor for this diagram. Performing the trace, $\operatorname{tr} t^{a} t^{b} t^{c}=\frac{1}{4}\left(d^{a b c}+i f^{a b c}\right)$, separates the $C$-odd contribution proportional to $d^{a b c}$ from the $C$-even contribution proportional to $i f^{a b c}$,

$$
\begin{aligned}
& \text { Fig. } 1\left(q_{1} g q_{1}\right)=-\frac{2 g^{5}}{3 \cdot 16 \pi^{3}} N_{c} \operatorname{tr} t^{b} t^{a} t^{c} \int\left[\mathrm{d} x_{i}\right] \int\left[\mathrm{d}^{2} k_{i}\right] \Psi_{q q q}\left(x_{1}, \vec{k}_{1} ; x_{2}, \vec{k}_{2} ; x_{3}, \vec{k}_{3}\right) \\
& \times \Psi_{q q q}^{*}\left(x_{1}, \vec{k}_{1}+x_{1} \vec{q}-\vec{q} ; x_{2}, \vec{k}_{2}+x_{2} \vec{q} ; x_{3}, \vec{k}_{3}+x_{3} \vec{q}\right) \\
& \times \frac{(2 \pi)^{D-1}}{2 p_{1}^{+}} \int \frac{\widetilde{\mathrm{d}}_{g}}{2\left(p_{1}^{+}-k_{g}^{+}\right)}\left\langle S\left|\hat{\psi}_{q \rightarrow q g}\left(\vec{p}_{1} ; \vec{p}_{1}-\vec{k}_{g}, \vec{k}_{g}\right) \hat{\psi}_{q \rightarrow q g}^{*}\left(\vec{p}_{1}-\vec{q} ; \vec{p}_{1}-\vec{k}_{g}-\vec{q}_{13}, \vec{k}_{g}-\vec{q}_{2}\right)\right| S\right\rangle .
\end{aligned}
$$

The symmetry factor is 3 .

$$
\begin{aligned}
\text { Fig.1 }\left(g q_{1} q_{1}\right)= & -\frac{2 g^{5}}{3 \cdot 16 \pi^{3}} N_{c} \operatorname{tr} t^{a} t^{b} t^{c} \int\left[\mathrm{d} x_{i}\right] \int\left[\mathrm{d}^{2} k_{i}\right] \Psi_{q q q}\left(x_{1}, \vec{k}_{1} ; x_{2}, \vec{k}_{2} ; x_{3}, \vec{k}_{3}\right) \\
& \times \Psi_{q q q}^{*}\left(x_{1}, \vec{k}_{1}+x_{1} \vec{q}-\vec{q} ; x_{2}, \vec{k}_{2}+x_{2} \vec{q} ; x_{3}, \vec{k}_{3}+x_{3} \vec{q}\right) \\
& \times \frac{(2 \pi)^{D-1}}{2 p_{1}^{+}} \int \frac{\widetilde{\mathrm{d}}_{g}}{2\left(p_{1}^{+}-k_{g}^{+}\right)}\left\langle S\left|\hat{\psi}_{q \rightarrow q g}\left(\vec{p}_{1} ; \vec{p}_{1}-\vec{k}_{g}, \vec{k}_{g}\right) \hat{\psi}_{q \rightarrow q g}^{*}\left(\vec{p}_{1}-\vec{q} ; \vec{p}_{1}-\vec{k}_{g}-\vec{q}_{12}, \vec{k}_{g}-\vec{q}_{3}\right)\right| S\right\rangle .
\end{aligned}
$$

The symmetry factor is 3 .

$$
\text { Fig. } 1\left(q_{1} g q_{2}\right)=0
$$

$$
\text { Fig. } 1\left(q_{2} q_{1} g\right)=0 \text {. }
$$

The symmetry factor is 6 , to include the contribution where the third gluon attaches to quark 3.

$$
\text { Fig. } 1\left(q_{1} q_{2} g\right)=0
$$

The symmetry factor is 6 .

$$
\text { Fig. } 1\left(q_{2} g q_{1}\right)=0 .
$$

The symmetry factor is 6 .
The symmetry factor is 6 .

$$
\text { Fig. } 1\left(g q_{2} q_{1}\right)=0 \text {. }
$$

The symmetry factor is 6 .

$$
\text { Fig. } 1\left(g q_{1} q_{2}\right)=0 \text {. }
$$

The symmetry factor is 6 .

$$
\begin{aligned}
& \text { Fig. } 1\left(q_{2} q_{2} g\right)=-\frac{2 g^{5}}{3 \cdot 16 \pi^{3}} \frac{1}{2} N_{c} \operatorname{tr} t^{a} t^{b} t^{c} \int\left[\mathrm{d} x_{i}\right] \int\left[\mathrm{d}^{2} k_{i}\right] \Psi_{q q q}\left(x_{1}, \vec{k}_{1} ; x_{2}, \vec{k}_{2} ; x_{3}, \vec{k}_{3}\right) \\
& \times \Psi_{q q q}^{*}\left(x_{1}, \vec{k}_{1}+x_{1} \vec{q}-\vec{q}_{1} ; x_{2}, \vec{k}_{2}+x_{2} \vec{q}-\vec{q}_{23} ; x_{3}, \vec{k}_{3}+x_{3} \vec{q}\right) \\
& \times \frac{(2 \pi)^{D-1}}{2 p_{1}^{+}} \int \frac{\widetilde{\mathrm{d} k}{ }_{g}}{2\left(p_{1}^{+}-k_{g}^{+}\right)}\left\langle S\left|\hat{\psi}_{q \rightarrow q g}\left(\vec{p}_{1} ; \vec{p}_{1}-\vec{k}_{g}, \vec{k}_{g}\right) \hat{\psi}_{q \rightarrow q g}^{*}\left(\vec{p}_{1}-\vec{q}_{1} ; \vec{p}_{1}-\vec{k}_{g}, \vec{k}_{g}-\vec{q}_{1}\right)\right| S\right\rangle .
\end{aligned}
$$


The symmetry factor is 6 .

$$
\begin{aligned}
& \text { Fig. } 1\left(q_{2} g q_{2}\right)=-\frac{2 g^{5}}{3 \cdot 16 \pi^{3}} \frac{1}{2} N_{c} \operatorname{tr} t^{a} t^{c} t^{b} \int\left[\mathrm{d} x_{i}\right] \int\left[\mathrm{d}^{2} k_{i}\right] \Psi_{q q q}\left(x_{1}, \vec{k}_{1} ; x_{2}, \vec{k}_{2} ; x_{3}, \vec{k}_{3}\right) \\
& \times \Psi_{q q q}^{*}\left(x_{1}, \vec{k}_{1}+x_{1} \vec{q}-\vec{q}_{2} ; x_{2}, \vec{k}_{2}+x_{2} \vec{q}-\vec{q}_{13} ; x_{3}, \vec{k}_{3}+x_{3} \vec{q}\right) \\
& \times \frac{(2 \pi)^{D-1}}{2 p_{1}^{+}} \int \frac{\widetilde{\mathrm{d}}_{g}}{2\left(p_{1}^{+}-k_{g}^{+}\right)}\left\langle S\left|\hat{\psi}_{q \rightarrow q g}\left(\vec{p}_{1} ; \vec{p}_{1}-\vec{k}_{g}, \vec{k}_{g}\right) \hat{\psi}_{q \rightarrow q g}^{*}\left(\vec{p}_{1}-\vec{q}_{2} ; \vec{p}_{1}-\vec{k}_{g}, \vec{k}_{g}-\vec{q}_{2}\right)\right| S\right\rangle .
\end{aligned}
$$

The symmetry factor is 6 .

$$
\begin{aligned}
& \text { Fig. } 1\left(g q_{2} q_{2}\right)=-\frac{2 g^{5}}{3 \cdot 16 \pi^{3}} \frac{1}{2} N_{c} \operatorname{tr} t^{a} t^{b} t^{c} \int\left[\mathrm{d} x_{i}\right] \int\left[\mathrm{d}^{2} k_{i}\right] \Psi_{q q q}\left(x_{1}, \vec{k}_{1} ; x_{2}, \vec{k}_{2} ; x_{3}, \vec{k}_{3}\right) \\
& \times \Psi_{q q q}^{*}\left(x_{1}, \vec{k}_{1}+x_{1} \vec{q}-\vec{q}_{3} ; x_{2}, \vec{k}_{2}+x_{2} \vec{q}-\vec{q}_{12} ; x_{3}, \vec{k}_{3}+x_{3} \vec{q}\right) \\
& \times \frac{(2 \pi)^{D-1}}{2 p_{1}^{+}} \int \frac{\widetilde{\mathrm{d}}_{g}}{2\left(p_{1}^{+}-k_{g}^{+}\right)}\left\langle S\left|\hat{\psi}_{q \rightarrow q g}\left(\vec{p}_{1} ; \vec{p}_{1}-\vec{k}_{g}, \vec{k}_{g}\right) \hat{\psi}_{q \rightarrow q g}^{*}\left(\vec{p}_{1}-\vec{q}_{3} ; \vec{p}_{1}-\vec{k}_{g}, \vec{k}_{g}-\vec{q}_{3}\right)\right| S\right\rangle .
\end{aligned}
$$

The symmetry factor is 6 .

$$
\begin{aligned}
& \text { Fig. } 1\left(q_{3} q_{2} g\right)=\frac{2 g^{5}}{3 \cdot 16 \pi^{3}} \frac{1}{2} N_{c} \operatorname{tr}\left(t^{a} t^{b} t^{c}+t^{a} t^{c} t^{b}\right) \int\left[\mathrm{d} x_{i}\right] \int\left[\mathrm{d}^{2} k_{i}\right] \Psi_{q q q}\left(x_{1}, \vec{k}_{1} ; x_{2}, \vec{k}_{2} ; x_{3}, \vec{k}_{3}\right) \\
& \times \Psi_{q q q}^{*}\left(x_{1}, \vec{k}_{1}+x_{1} \vec{q}-\vec{q}_{1} ; x_{2}, \vec{k}_{2}+x_{2} \vec{q}-\vec{q}_{2} ; x_{3}, \vec{k}_{3}+x_{3} \vec{q}-\vec{q}_{3}\right) \\
& \times \frac{(2 \pi)^{D-1}}{2 p_{1}^{+}} \int \frac{\widetilde{\mathrm{d}}_{g}}{2\left(p_{1}^{+}-k_{g}^{+}\right)}\left\langle S\left|\hat{\psi}_{q \rightarrow q g}\left(\vec{p}_{1} ; \vec{p}_{1}-\vec{k}_{g}, \vec{k}_{g}\right) \hat{\psi}_{q \rightarrow q g}^{*}\left(\vec{p}_{1}-\vec{q}_{1} ; \vec{p}_{1}-\vec{k}_{g}, \vec{k}_{g}-\vec{q}_{1}\right)\right| S\right\rangle .
\end{aligned}
$$

The symmetry factor is 6 .

$$
\begin{aligned}
& \text { Fig. } 1\left(q_{3} g q_{2}\right)=\frac{2 g^{5}}{3 \cdot 16 \pi^{3}} \frac{1}{2} N_{c} \operatorname{tr}\left(t^{a} t^{b} t^{c}+t^{a} t^{c} t^{b}\right) \int\left[\mathrm{d} x_{i}\right] \int\left[\mathrm{d}^{2} k_{i}\right] \Psi_{q q q}\left(x_{1}, \vec{k}_{1} ; x_{2}, \vec{k}_{2} ; x_{3}, \vec{k}_{3}\right) \\
& \times \Psi_{q q q}^{*}\left(x_{1}, \vec{k}_{1}+x_{1} \vec{q}-\vec{q}_{2} ; x_{2}, \vec{k}_{2}+x_{2} \vec{q}-\vec{q}_{1} ; x_{3}, \vec{k}_{3}+x_{3} \vec{q}-\vec{q}_{3}\right) \\
& \times \frac{(2 \pi)^{D-1}}{2 p_{1}^{+}} \int \frac{\widetilde{\mathrm{d}}_{g}}{2\left(p_{1}^{+}-k_{g}^{+}\right)}\left\langle S\left|\hat{\psi}_{q \rightarrow q g}\left(\vec{p}_{1} ; \vec{p}_{1}-\vec{k}_{g}, \vec{k}_{g}\right) \hat{\psi}_{q \rightarrow q g}^{*}\left(\vec{p}_{1}-\vec{q}_{2} ; \vec{p}_{1}-\vec{k}_{g}, \vec{k}_{g}-\vec{q}_{2}\right)\right| S\right\rangle .
\end{aligned}
$$

The symmetry factor is 6 .

$$
\begin{aligned}
& \text { Fig. } 1\left(g q_{3} q_{2}\right)=\frac{2 g^{5}}{3 \cdot 16 \pi^{3}} \frac{1}{2} N_{c} \operatorname{tr}\left(t^{a} t^{b} t^{c}+t^{a} t^{c} t^{b}\right) \int\left[\mathrm{d} x_{i}\right] \int\left[\mathrm{d}^{2} k_{i}\right] \Psi_{q q q}\left(x_{1}, \vec{k}_{1} ; x_{2}, \vec{k}_{2} ; x_{3}, \vec{k}_{3}\right) \\
& \times \Psi_{q q q}^{*}\left(x_{1}, \vec{k}_{1}+x_{1} \vec{q}-\vec{q}_{3} ; x_{2}, \vec{k}_{2}+x_{2} \vec{q}-\vec{q}_{1} ; x_{3}, \vec{k}_{3}+x_{3} \vec{q}-\vec{q}_{2}\right) \\
& \times \frac{(2 \pi)^{D-1}}{2 p_{1}^{+}} \int \frac{\widetilde{\mathrm{d}}_{g}}{2\left(p_{1}^{+}-k_{g}^{+}\right)}\left\langle S\left|\hat{\psi}_{q \rightarrow q g}\left(\vec{p}_{1} ; \vec{p}_{1}-\vec{k}_{g}, \vec{k}_{g}\right) \hat{\psi}_{q \rightarrow q g}^{*}\left(\vec{p}_{1}-\vec{q}_{3} ; \vec{p}_{1}-\vec{k}_{g}, \vec{k}_{g}-\vec{q}_{3}\right)\right| S\right\rangle .
\end{aligned}
$$

The symmetry factor is 6 .

We now proceed to the diagrams where all three gluon probes couple to quarks. First, there is the expectation value of $\rho_{\mathrm{qu}}\left(\vec{q}_{1}\right) \rho_{\mathrm{qu}}\left(\vec{q}_{2}\right) \rho_{\mathrm{qu}}\left(\vec{q}_{3}\right)$ between $|q q q\rangle$ three quark states:

$$
\begin{aligned}
\left\langle\rho^{a}\left(\vec{q}_{1}\right) \rho^{b}\left(\vec{q}_{2}\right) \rho^{c}\left(\vec{q}_{3}\right)\right\rangle= & \frac{g^{3}}{6} \int\left[\mathrm{d} x_{i}\right] \int\left[\mathrm{d}^{2} k_{i}\right]\left[\operatorname{tr} t^{a} t^{b} t^{c} \Psi^{*}\left(x_{1}, \vec{k}_{1}-\vec{q}+x_{1} q ; x_{2}, \vec{k}_{2}+x_{2} \vec{q} ; x_{3}, \vec{k}_{3}+x_{3} \vec{q}\right)\right. \\
& -\operatorname{tr} t^{a} t^{b} t^{c} \Psi^{*}\left(x_{1}, \vec{k}_{1}-\vec{q}_{1}+x_{1} \vec{q} ; x_{2}, \vec{k}_{2}-\vec{q}_{23}+x_{2} \vec{q} ; x_{3}, \vec{k}_{3}+x_{3} \vec{q}\right) \\
& -\operatorname{tr} t^{a} t^{c} t^{b} \Psi^{*}\left(x_{1}, \vec{k}_{1}-\vec{q}_{13}+x_{1} \vec{q} ; x_{2}, \vec{k}_{2}-\vec{q}_{2}+x_{2} \vec{q} ; x_{3}, \vec{k}_{3}+x_{3} \vec{q}\right) \\
& -\operatorname{tr} t^{a} t^{b} t^{c} \Psi^{*}\left(x_{1}, \vec{k}_{1}-\vec{q}_{12}+x_{1} \vec{q} ; x_{2}, \vec{k}_{2}-\vec{q}_{3}+x_{2} \vec{q} ; x_{3}, \vec{k}_{3}+x_{3} \vec{q}\right) \\
& \left.+\left(\operatorname{tr} t^{a} t^{b} t^{c}+\operatorname{tr} t^{a} t^{c} t^{b}\right) \Psi^{*}\left(x_{1}, \vec{k}_{1}-\vec{q}_{1}+x_{1} \vec{q} ; x_{2}, \vec{k}_{2}-\vec{q}_{2}+x_{2} \vec{q} ; x_{3}, \vec{k}_{3}-\vec{q}_{3}+x_{3} \vec{q}\right)\right] \\
& \times \psi\left(x_{1}, \vec{k}_{1} ; x_{2}, \vec{k}_{2} ; x_{3}, \vec{k}_{3}\right) .
\end{aligned}
$$



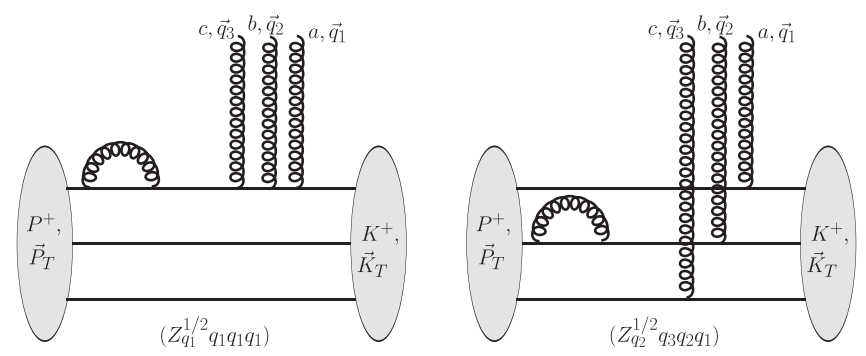

FIG. 2. Two of the diagrams for $\left\langle\rho_{\mathrm{qu}}^{a}\left(\vec{q}_{1}\right) \rho_{\mathrm{qu}}^{b}\left(\vec{q}_{2}\right) \rho_{\mathrm{qu}}^{c}\left(\vec{q}_{3}\right)\right\rangle$ in the three-quark Fock state which involve the quark wave function renormalization factor $Z_{q}^{1 / 2}(x)$ ("virtual corrections"). The cut is located at the insertion of the three color charge operators.

The first to fifth terms, respectively, correspond to Fig.2( $\left.q_{1} q_{1} q_{1}\right)$, Fig. $2\left(q_{2} q_{2} q_{1}\right)+$ Fig. $2\left(q_{3} q_{3} q_{1}\right)$, Fig. $2\left(q_{1} q_{2} q_{1}\right)+$ Fig. $2\left(q_{1} q_{3} q_{1}\right)$, Fig. $2\left(q_{2} q_{1} q_{1}\right)+$ Fig. $2\left(q_{3} q_{1} q_{1}\right)$, Fig. $2\left(q_{3} q_{2} q_{1}\right)+$ Fig, $2\left(q_{2} q_{3} q_{1}\right)$. Two example diagrams (first and seventh term in the previous expression) are shown in Fig. 2. The entire expression comes with a symmetry factor of 3. To account for the quark wave function renormalization factor, we multiply it by $Z_{q}\left(x_{1}\right) Z_{q}\left(x_{2}\right) Z_{q}\left(x_{3}\right)=1-$ $C_{q}\left(x_{1}\right)-C_{q}\left(x_{2}\right)-C_{q}\left(x_{3}\right)$ where $C_{q}(x)=\mathcal{O}\left(g^{2}\right)$. The explicit expression for $C_{q}(x)$ in the $\overline{M S}$ scheme is given in Ref. [1] but is not needed here because we will verify that all UV divergences cancel.

The final set of UV divergent diagrams is shown in Fig. 3. Here, quark 1 exchanges a gluon with itself across the insertion of the three charge operators while the three gluon probes attach in all possible ways to the three quarks,

$$
\begin{aligned}
\text { Fig. } 3\left(q_{1} q_{1} q_{1}\right)= & \frac{2 g^{5}}{3 \cdot 16 \pi^{3}} 2 C_{F} \operatorname{tr} t^{a} t^{b} t^{c} \int\left[\mathrm{d} x_{i}\right] \int\left[\mathrm{d}^{2} k_{i}\right] \Psi_{q q q}\left(x_{1}, \vec{k}_{1} ; x_{2}, \vec{k}_{2} ; x_{3}, \vec{k}_{3}\right) \\
& \times \Psi_{q q q}^{*}\left(x_{1}, \vec{k}_{1}-\left(1-x_{1}\right) \vec{q} ; x_{2}, \vec{k}_{2}+x_{2} \vec{q} ; x_{3}, \vec{k}_{3}+x_{3} \vec{q}\right) \\
& \times \frac{(2 \pi)^{D-1}}{2 p_{1}^{+}} \int \frac{\widetilde{\mathrm{d} k_{g}}}{2\left(p_{1}^{+}-k_{g}^{+}\right)}\left\langle S\left|\hat{\psi}_{q \rightarrow q g}\left(\vec{p}_{1} ; \vec{p}_{1}-\vec{k}_{g}, \vec{k}_{g}\right) \hat{\psi}_{q \rightarrow q g}^{*}\left(\vec{p}_{1}-\vec{q} ; \vec{p}_{1}-\overrightarrow{k_{g}}-\vec{q}, \vec{k}_{g}\right)\right| S\right\rangle,
\end{aligned}
$$

with a symmetry factor of 3 .



FIG. 3. Final set of UV divergent "real emission" diagrams for $\left\langle\rho^{a}\left(\vec{q}_{1}\right) \rho^{b}\left(\vec{q}_{2}\right) \rho^{c}\left(\vec{q}_{3}\right)\right\rangle$ where all three gluon probes attach to quarks in the proton. The cut is located at the insertion of the three color charge operators. 
Fig. $3\left(q_{2} q_{1} q_{1}\right)=\frac{2 g^{5}}{3 \cdot 16 \pi^{3}} \frac{1}{2 N_{c}} \operatorname{tr} t^{a} t^{b} t^{c} \int\left[\mathrm{d} x_{i}\right] \int\left[\mathrm{d}^{2} k_{i}\right] \Psi_{q q q}\left(x_{1}, \vec{k}_{1} ; x_{2}, \vec{k}_{2} ; x_{3}, \vec{k}_{3}\right)$

$$
\begin{aligned}
& \times \Psi_{q q q}^{*}\left(x_{1}, \vec{k}_{1}+x_{1} \vec{q}-\vec{q}_{12} ; x_{2}, \vec{k}_{2}+x_{2} \vec{q}-\vec{q}_{3} ; x_{3}, \vec{k}_{3}+x_{3} \vec{q}\right) \\
& \times \frac{(2 \pi)^{D-1}}{2 p_{1}^{+}} \int \frac{\widetilde{\mathrm{d}} k_{g}}{2\left(p_{1}^{+}-k_{g}^{+}\right)}\left\langle S\left|\hat{\psi}_{q \rightarrow q g}\left(\vec{p}_{1} ; \vec{p}_{1}-\vec{k}_{g}, \vec{k}_{g}\right) \hat{\psi}_{q \rightarrow q g}^{*}\left(\vec{p}_{1}-\vec{q}_{12} ; \vec{p}_{1}-\vec{k}_{g}-\vec{q}_{12}, \vec{k}_{g}\right)\right| S\right\rangle,
\end{aligned}
$$

with a symmetry factor of 6 .

$$
\begin{aligned}
\text { Fig. } 3\left(q_{1} q_{2} q_{1}\right)= & \frac{2 g^{5}}{3 \cdot 16 \pi^{3}} \frac{1}{2 N_{c}} \operatorname{tr} t^{a} t^{c} t^{b} \int\left[\mathrm{d} x_{i}\right] \int\left[\mathrm{d}^{2} k_{i}\right] \Psi_{q q q}\left(x_{1}, \vec{k}_{1} ; x_{2}, \vec{k}_{2} ; x_{3}, \vec{k}_{3}\right) \\
& \times \Psi_{q q q}^{*}\left(x_{1}, \vec{k}_{1}+x_{1} \vec{q}-\vec{q}_{13} ; x_{2}, \vec{k}_{2}+x_{2} \vec{q}-\vec{q}_{2} ; x_{3}, \vec{k}_{3}+x_{3} \vec{q}\right) \\
& \times \frac{(2 \pi)^{D-1}}{2 p_{1}^{+}} \int \frac{\widetilde{\mathrm{d} k_{g}}}{2\left(p_{1}^{+}-k_{g}^{+}\right)}\left\langle S\left|\hat{\psi}_{q \rightarrow q g}\left(\vec{p}_{1} ; \vec{p}_{1}-\vec{k}_{g}, \vec{k}_{g}\right) \hat{\psi}_{q \rightarrow q g}^{*}\left(\vec{p}_{1}-\vec{q}_{13} ; \vec{p}_{1}-\vec{k}_{g}-\vec{q}_{13}, \vec{k}_{g}\right)\right| S\right\rangle,
\end{aligned}
$$

with a symmetry factor of 6 .

$$
\begin{aligned}
\text { Fig. } 3\left(q_{2} q_{2} q_{1}\right)= & \frac{2 g^{5}}{3 \cdot 16 \pi^{3}} \frac{1}{2 N_{c}} \operatorname{tr} t^{a} t^{b} t^{c} \int\left[\mathrm{d} x_{i}\right] \int\left[\mathrm{d}^{2} k_{i}\right] \Psi_{q q q}\left(x_{1}, \vec{k}_{1} ; x_{2}, \vec{k}_{2} ; x_{3}, \vec{k}_{3}\right) \\
& \times \Psi_{q q q}^{*}\left(x_{1}, \vec{k}_{1}+x_{1} \vec{q}-\vec{q}_{1} ; x_{2}, \vec{k}_{2}+x_{2} \vec{q}-\vec{q}_{23} ; x_{3}, \vec{k}_{3}+x_{3} \vec{q}\right) \\
& \times \frac{(2 \pi)^{D-1}}{2 p_{1}^{+}} \int \frac{\widetilde{\mathrm{d}}_{g}}{2\left(p_{1}^{+}-k_{g}^{+}\right)}\left\langle S\left|\hat{\psi}_{q \rightarrow q g}\left(\vec{p}_{1} ; \vec{p}_{1}-\vec{k}_{g}, \vec{k}_{g}\right) \hat{\psi}_{q \rightarrow q g}^{*}\left(\vec{p}_{1}-\vec{q}_{1} ; \vec{p}_{1}-\vec{k}_{g}-\vec{q}_{1}, \vec{k}_{g}\right)\right| S\right\rangle,
\end{aligned}
$$

with a symmetry factor of 6 .

$$
\begin{aligned}
& \text { Fig. } 3\left(q_{3} q_{2} q_{1}\right)=-\frac{2 g^{5}}{3 \cdot 16 \pi^{3}} \frac{1}{2 N_{c}}\left(\operatorname{tr} t^{a} t^{b} t^{c}+\operatorname{tr} t^{a} t^{c} t^{b}\right) \int\left[\mathrm{d} x_{i}\right] \int\left[\mathrm{d}^{2} k_{i}\right] \Psi_{q q q}\left(x_{1}, \vec{k}_{1} ; x_{2}, \vec{k}_{2} ; x_{3}, \vec{k}_{3}\right) \\
& \times \Psi_{q q q}^{*}\left(x_{1}, \vec{k}_{1}+x_{1} \vec{q}-\vec{q}_{1} ; x_{2}, \vec{k}_{2}+x_{2} \vec{q}-\vec{q}_{2} ; x_{3}, \vec{k}_{3}+x_{3} \vec{q}-\vec{q}_{3}\right) \\
& \times \frac{(2 \pi)^{D-1}}{2 p_{1}^{+}} \int \frac{\widetilde{\mathrm{d}} k_{g}}{2\left(p_{1}^{+}-k_{g}^{+}\right)}\left\langle S\left|\hat{\psi}_{q \rightarrow q g}\left(\vec{p}_{1} ; \vec{p}_{1}-\vec{k}_{g}, \vec{k}_{g}\right) \hat{\psi}_{q \rightarrow q g}^{*}\left(\vec{p}_{1}-\vec{q}_{1} ; \vec{p}_{1}-\vec{k}_{g}-\vec{q}_{1}, \vec{k}_{g}\right)\right| S\right\rangle,
\end{aligned}
$$

with a symmetry factor of 6 .

$$
\begin{aligned}
\text { Fig. } 3\left(q_{1} q_{1} q_{2}\right)= & \frac{2 g^{5}}{3 \cdot 16 \pi^{3}} \frac{1}{2 N_{c}} \operatorname{tr} t^{a} t^{b} t^{c} \int\left[\mathrm{d} x_{i}\right] \int\left[\mathrm{d}^{2} k_{i}\right] \Psi_{q q q}\left(x_{1}, \vec{k}_{1} ; x_{2}, \vec{k}_{2} ; x_{3}, \vec{k}_{3}\right) \\
& \times \Psi_{q q q}^{*}\left(x_{1}, \vec{k}_{1}+x_{1} \vec{q}-\vec{q}_{23} ; x_{2}, \vec{k}_{2}+x_{2} \vec{q}-\vec{q}_{1} ; x_{3}, \vec{k}_{3}+x_{3} \vec{q}\right) \\
& \times \frac{(2 \pi)^{D-1}}{2 p_{1}^{+}} \int \frac{\widetilde{\mathrm{d} k_{g}}}{2\left(p_{1}^{+}-k_{g}^{+}\right)}\left\langle S\left|\hat{\psi}_{q \rightarrow q g}\left(\vec{p}_{1} ; \vec{p}_{1}-\vec{k}_{g}, \vec{k}_{g}\right) \hat{\psi}_{q \rightarrow q g}^{*}\left(\vec{p}_{1}-\vec{q}_{23} ; \vec{p}_{1}-\vec{k}_{g}-\vec{q}_{23}, \vec{k}_{g}\right)\right| S\right\rangle,
\end{aligned}
$$

with a symmetry factor of 6 .

$$
\begin{aligned}
& \text { Fig. } 3\left(q_{2} q_{1} q_{2}\right)=\frac{2 g^{5}}{3 \cdot 16 \pi^{3}} \frac{1}{2 N_{c}} \operatorname{tr}^{a} t^{c} t^{b} \int\left[\mathrm{d} x_{i}\right] \int\left[\mathrm{d}^{2} k_{i}\right] \Psi_{q q q}\left(x_{1}, \vec{k}_{1} ; x_{2}, \vec{k}_{2} ; x_{3}, \vec{k}_{3}\right) \\
& \times \Psi_{q q q}^{*}\left(x_{1}, \vec{k}_{1}+x_{1} \vec{q}-\vec{q}_{2} ; x_{2}, \vec{k}_{2}+x_{2} \vec{q}-\vec{q}_{13} ; x_{3}, \vec{k}_{3}+x_{3} \vec{q}\right) \\
& \times \frac{(2 \pi)^{D-1}}{2 p_{1}^{+}} \int \frac{\widetilde{\mathrm{d} k}{ }_{g}}{2\left(p_{1}^{+}-k_{g}^{+}\right)}\left\langle S\left|\hat{\psi}_{q \rightarrow q g}\left(\vec{p}_{1} ; \vec{p}_{1}-\vec{k}_{g}, \vec{k}_{g}\right) \hat{\psi}_{q \rightarrow q g}^{*}\left(\vec{p}_{1}-\vec{q}_{2} ; \vec{p}_{1}-\vec{k}_{g}-\vec{q}_{2}, \vec{k}_{g}\right)\right| S\right\rangle \text {, }
\end{aligned}
$$

with a symmetry factor of 6 . 


$$
\begin{aligned}
& \text { Fig. } 3\left(q_{1} q_{2} q_{2}\right)=\frac{2 g^{5}}{3 \cdot 16 \pi^{3}} \frac{1}{2 N_{c}} \operatorname{tr} t^{a} t^{b} t^{c} \int\left[\mathrm{d} x_{i}\right] \int\left[\mathrm{d}^{2} k_{i}\right] \Psi_{q q q}\left(x_{1}, \vec{k}_{1} ; x_{2}, \vec{k}_{2} ; x_{3}, \vec{k}_{3}\right) \\
& \times \Psi_{q q q}^{*}\left(x_{1}, \vec{k}_{1}+x_{1} \vec{q}-\vec{q}_{3} ; x_{2}, \vec{k}_{2}+x_{2} \vec{q}-\vec{q}_{12} ; x_{3}, \vec{k}_{3}+x_{3} \vec{q}\right) \\
& \times \frac{(2 \pi)^{D-1}}{2 p_{1}^{+}} \int \frac{\widetilde{\mathrm{d}}_{g}}{2\left(p_{1}^{+}-k_{g}^{+}\right)}\left\langle S\left|\hat{\psi}_{q \rightarrow q g}\left(\vec{p}_{1} ; \vec{p}_{1}-\vec{k}_{g}, \vec{k}_{g}\right) \hat{\psi}_{q \rightarrow q g}^{*}\left(\vec{p}_{1}-\vec{q}_{3} ; \vec{p}_{1}-\vec{k}_{g}-\vec{q}_{3}, \vec{k}_{g}\right)\right| S\right\rangle,
\end{aligned}
$$

with a symmetry factor of 6 .

$$
\begin{aligned}
& \text { Fig. } 3\left(q_{2} q_{2} q_{2}\right)=\frac{2 g^{5}}{3 \cdot 16 \pi^{3}} C_{F}\left(N_{c}-1\right) \operatorname{tr} t^{a} t^{b} t^{c} \int\left[\mathrm{d} x_{i}\right] \int\left[\mathrm{d}^{2} k_{i}\right] \Psi_{q q q}\left(x_{1}, \vec{k}_{1} ; x_{2}, \vec{k}_{2} ; x_{3}, \vec{k}_{3}\right) \\
& \times \Psi_{q q q}^{*}\left(x_{1}, \vec{k}_{1}+x_{1} \vec{q} ; x_{2}, \vec{k}_{2}+x_{2} \vec{q}-\vec{q} ; x_{3}, \vec{k}_{3}+x_{3} \vec{q}\right) \\
& \times \frac{(2 \pi)^{D-1}}{2 p_{1}^{+}} \int \frac{\widetilde{\mathrm{d}} k_{g}}{2\left(p_{1}^{+}-k_{g}^{+}\right)}\left\langle S\left|\hat{\psi}_{q \rightarrow q g}\left(\vec{p}_{1} ; \vec{p}_{1}-\vec{k}_{g}, \vec{k}_{g}\right) \hat{\psi}_{q \rightarrow q g}^{*}\left(\vec{p}_{1} ; \vec{p}_{1}-\vec{k}_{g}, \vec{k}_{g}\right)\right| S\right\rangle,
\end{aligned}
$$

with a symmetry factor of 6 .

$$
\begin{aligned}
& \text { Fig. } 3\left(q_{3} q_{2} q_{2}\right)=-\frac{2 g^{5}}{3 \cdot 16 \pi^{3}}\left(N_{c}-2\right) C_{F} \operatorname{tr} t^{a} t^{b} t^{c} \int\left[\mathrm{d} x_{i}\right] \int\left[\mathrm{d}^{2} k_{i}\right] \Psi_{q q q}\left(x_{1}, \vec{k}_{1} ; x_{2}, \vec{k}_{2} ; x_{3}, \vec{k}_{3}\right) \\
& \times \Psi_{q q q}^{*}\left(x_{1}, \vec{k}_{1}+x_{1} \vec{q} ; x_{2}, \vec{k}_{2}+x_{2} \vec{q}-\vec{q}_{12} ; x_{3}, \vec{k}_{3}+x_{3} \vec{q}-\vec{q}_{3}\right) \\
& \times \frac{(2 \pi)^{D-1}}{2 p_{1}^{+}} \int \frac{\widetilde{\mathrm{d} k}}{2\left(p_{1}^{+}-k_{g}^{+}\right)}\left\langle S\left|\hat{\psi}_{q \rightarrow q g}\left(\vec{p}_{1} ; \vec{p}_{1}-\vec{k}_{g}, \vec{k}_{g}\right) \hat{\psi}_{q \rightarrow q g}^{*}\left(\vec{p}_{1} ; \vec{p}_{1}-\vec{k}_{g}, \vec{k}_{g}\right)\right| S\right\rangle,
\end{aligned}
$$

with a symmetry factor of 6 .

$$
\begin{aligned}
& \text { Fig. } 3\left(q_{2} q_{3} q_{2}\right)=-\frac{2 g^{5}}{3 \cdot 16 \pi^{3}}\left(N_{c}-2\right) C_{F} \operatorname{tr} t^{a} t^{c} t^{b} \int\left[\mathrm{d} x_{i}\right] \int\left[\mathrm{d}^{2} k_{i}\right] \Psi_{q q q}\left(x_{1}, \vec{k}_{1} ; x_{2}, \vec{k}_{2} ; x_{3}, \vec{k}_{3}\right) \\
& \times \Psi_{q q q}^{*}\left(x_{1}, \vec{k}_{1}+x_{1} \vec{q} ; x_{2}, \vec{k}_{2}+x_{2} \vec{q}-\vec{q}_{13} ; x_{3}, \vec{k}_{3}+x_{3} \vec{q}-\vec{q}_{2}\right) \\
& \times \frac{(2 \pi)^{D-1}}{2 p_{1}^{+}} \int \frac{\widetilde{\mathrm{d}}_{g}}{2\left(p_{1}^{+}-k_{g}^{+}\right)}\left\langle S\left|\hat{\psi}_{q \rightarrow q g}\left(\vec{p}_{1} ; \vec{p}_{1}-\vec{k}_{g}, \vec{k}_{g}\right) \hat{\psi}_{q \rightarrow q g}^{*}\left(\vec{p}_{1} ; \vec{p}_{1}-\vec{k}_{g}, \vec{k}_{g}\right)\right| S\right\rangle,
\end{aligned}
$$

with a symmetry factor of 6 .

$$
\begin{aligned}
& \text { Fig. } 3\left(q_{3} q_{3} q_{2}\right)=-\frac{2 g^{5}}{3 \cdot 16 \pi^{3}}\left(N_{c}-2\right) C_{F} \operatorname{tr} t^{a} t^{b} t^{c} \int\left[\mathrm{d} x_{i}\right] \int\left[\mathrm{d}^{2} k_{i}\right] \Psi_{q q q}\left(x_{1}, \vec{k}_{1} ; x_{2}, \vec{k}_{2} ; x_{3}, \vec{k}_{3}\right) \\
& \times \Psi_{q q q}^{*}\left(x_{1}, \vec{k}_{1}+x_{1} \vec{q} ; x_{2}, \vec{k}_{2}+x_{2} \vec{q}-\vec{q}_{1} ; x_{3}, \vec{k}_{3}+x_{3} \vec{q}-\vec{q}_{23}\right) \\
& \times \frac{(2 \pi)^{D-1}}{2 p_{1}^{+}} \int \frac{\widetilde{\mathrm{d}}_{g}}{2\left(p_{1}^{+}-k_{g}^{+}\right)}\left\langle S\left|\hat{\psi}_{q \rightarrow q g}\left(\vec{p}_{1} ; \vec{p}_{1}-\vec{k}_{g}, \vec{k}_{g}\right) \hat{\psi}_{q \rightarrow q g}^{*}\left(\vec{p}_{1} ; \vec{p}_{1}-\vec{k}_{g}, \vec{k}_{g}\right)\right| S\right\rangle,
\end{aligned}
$$

with a symmetry factor of 6 .

$$
\begin{aligned}
& \text { Fig. } 3\left(q_{3} q_{1} q_{2}\right)=-\frac{2 g^{5}}{3 \cdot 16 \pi^{3}} \frac{1}{2 N_{c}}\left(\operatorname{tr} t^{a} t^{b} t^{c}+\operatorname{tr} t^{a} t^{c} t^{b}\right) \int\left[\mathrm{d} x_{i}\right] \int\left[\mathrm{d}^{2} k_{i}\right] \Psi_{q q q}\left(x_{1}, \vec{k}_{1} ; x_{2}, \vec{k}_{2} ; x_{3}, \vec{k}_{3}\right) \\
& \times \Psi_{q q q}^{*}\left(x_{1}, \vec{k}_{1}+x_{1} \vec{q}-\vec{q}_{2} ; x_{2}, \vec{k}_{2}+x_{2} \vec{q}-\vec{q}_{1} ; x_{3}, \vec{k}_{3}+x_{3} \vec{q}-\vec{q}_{3}\right) \\
& \times \frac{(2 \pi)^{D-1}}{2 p_{1}^{+}} \int \frac{\widetilde{\mathrm{d}}_{g}}{2\left(p_{1}^{+}-k_{g}^{+}\right)}\left\langle S\left|\hat{\psi}_{q \rightarrow q g}\left(\vec{p}_{1} ; \vec{p}_{1}-\vec{k}_{g}, \vec{k}_{g}\right) \hat{\psi}_{q \rightarrow q g}^{*}\left(\vec{p}_{1}-\vec{q}_{2} ; \vec{p}_{1}-\vec{k}_{g}-\vec{q}_{2}, \vec{k}_{g}\right)\right| S\right\rangle,
\end{aligned}
$$

with a symmetry factor of 6 . 


$$
\begin{aligned}
& \text { Fig. } 3\left(q_{1} q_{3} q_{2}\right)=-\frac{2 g^{5}}{3 \cdot 16 \pi^{3}} \frac{1}{2 N_{c}}\left(\operatorname{tr} t^{a} t^{b} t^{c}+\operatorname{tr}^{a} t^{c} t^{b}\right) \int\left[\mathrm{d} x_{i}\right] \int\left[\mathrm{d}^{2} k_{i}\right] \Psi_{q q q}\left(x_{1}, \vec{k}_{1} ; x_{2}, \vec{k}_{2} ; x_{3}, \vec{k}_{3}\right) \\
& \times \Psi_{q q q}^{*}\left(x_{1}, \vec{k}_{1}+x_{1} \vec{q}-\vec{q}_{3} ; x_{2}, \vec{k}_{2}+x_{2} \vec{q}-\vec{q}_{1} ; x_{3}, \vec{k}_{3}+x_{3} \vec{q}-\vec{q}_{2}\right) \\
& \times \frac{(2 \pi)^{D-1}}{2 p_{1}^{+}} \int \frac{\widetilde{\mathrm{d}}_{g}}{2\left(p_{1}^{+}-k_{g}^{+}\right)}\left\langle S\left|\hat{\psi}_{q \rightarrow q g}\left(\vec{p}_{1} ; \vec{p}_{1}-\vec{k}_{g}, \vec{k}_{g}\right) \hat{\psi}_{q \rightarrow q g}^{*}\left(\vec{p}_{1}-\vec{q}_{3} ; \vec{p}_{1}-\vec{k}_{g}-\vec{q}_{3}, \vec{k}_{g}\right)\right| S\right\rangle,
\end{aligned}
$$

with a symmetry factor of 6 .

\section{B. Finite diagrams}

\section{Coupling at least once to the gluon}

We now proceed to the UV-finite contributions. To write the following expressions in a more compact form we introduce the integral operator

$$
\begin{aligned}
\vec{I}= & \frac{g^{5}}{3 \cdot 16 \pi^{3}} \int\left[\mathrm{d} x_{i}\right] \int\left[\mathrm{d}^{2} k_{i}\right] \Psi_{q q q}\left(x_{1}, \vec{k}_{1} ; x_{2}, \vec{k}_{2} ; x_{3}, \vec{k}_{3}\right) \\
& \times \int_{x}^{\min \left(x_{1}, 1-x_{2}\right)} \frac{\mathrm{d} x_{g}}{x_{g}}\left(1-\frac{z_{1}+z_{2}}{2}+\frac{z_{1} z_{2}}{6}\right) \sqrt{\frac{x_{1}}{x_{1}-x_{g}}} \sqrt{\frac{x_{2}}{x_{2}+x_{g}}} \int \mathrm{d}^{2} k_{g} \frac{z_{1} \vec{p}_{1}-\vec{k}_{g}}{\left(z_{1} \vec{p}_{1}-\vec{k}_{g}\right)^{2}},
\end{aligned}
$$

with $z_{1}=x_{g} / x_{1}$ and $z_{2}=x_{g} /\left(x_{2}+x_{g}\right)$. We begin with the diagrams shown in Fig. 4 where two distinct quarks exchange a gluon across the operator insertion, and where two of the probes attach to that gluon,

$$
\begin{aligned}
\text { Fig.4(ggg) }= & -\frac{1}{2} \operatorname{tr} T^{a} T^{b} T^{c} \vec{I} \cdot \frac{z_{2} \vec{p}_{2}-\left(1-z_{2}\right)\left(\vec{k}_{g}-\vec{q}\right)}{\left(z_{2} \vec{p}_{2}-\left(1-z_{2}\right)\left(\vec{k}_{g}-\vec{q}\right)\right)^{2}} \\
& \times \Psi_{q q q}^{*}\left(x_{1}-x_{g}, \vec{k}_{1}+x_{1} \vec{q}-\vec{k}_{g}+x_{g} \vec{K} ; x_{2}+x_{g}, \vec{k}_{2}-\left(1-x_{2}\right) \vec{q}+\vec{k}_{g}-x_{g} \vec{K} ; x_{3}, \vec{k}_{3}+x_{3} \vec{q}\right) .
\end{aligned}
$$

The symmetry factor is 6 which includes a factor of 2 for interchanging the gluon emission and absorption vertices between quarks 1 and 2 (so that in $|P\rangle$ the gluon couples to quark 2, and in $\langle K|$ it couples to quark 1),

$$
\begin{aligned}
\text { Fig.4(q } \left.q_{1} g g\right)= & -\frac{1}{4} \operatorname{tr}\left(T^{a} T^{b} D^{c}-T^{a} T^{b} T^{c}\right) \vec{I} \cdot \frac{z_{2} \vec{p}_{2}-\left(1-z_{2}\right)\left(\vec{k}_{g}-\vec{q}_{12}\right)}{\left(z_{2} \vec{p}_{2}-\left(1-z_{2}\right)\left(\vec{k}_{g}-\vec{q}_{12}\right)\right)^{2}} \\
& \times \Psi_{q q q}^{*}\left(x_{1}-x_{g}, \vec{k}_{1}+x_{1} \vec{q}-\vec{q}_{3}-\vec{k}_{g}+x_{g} \vec{K} ; x_{2}+x_{g}, \vec{k}_{2}+x_{2} \vec{q}-\vec{q}_{12}+\vec{k}_{g}-x_{g} \vec{K} ; x_{3}, \vec{k}_{3}+x_{3} \vec{q}\right) .
\end{aligned}
$$

The symmetry factor of 6 includes a factor of 2 due to the contribution from Fig.4 $\left(q_{2} g g\right)$ with the gluon emission and absorption vertices between quarks 1 and 2 interchanged,
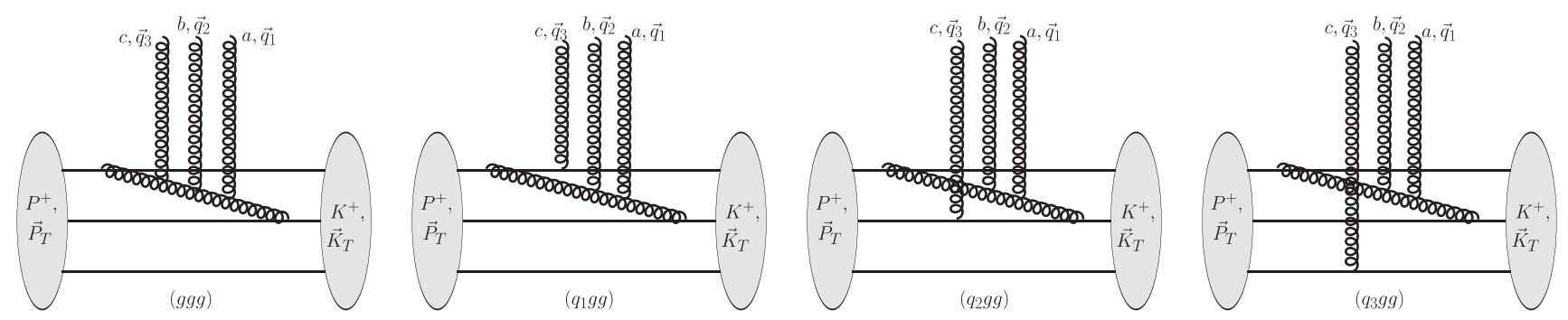

FIG. 4. A sample of UV finite diagrams for $\left\langle\rho^{a}\left(\vec{q}_{1}\right) \rho^{b}\left(\vec{q}_{2}\right) \rho^{c}\left(\vec{q}_{3}\right)\right\rangle$ where at least two of the probes attach to the gluon in the proton. The cut is located at the insertion of the three color charge operators. 
Fig. $4\left(g q_{1} g\right)=-\frac{1}{4} \operatorname{tr}\left(T^{a} T^{c} D^{b}-T^{a} T^{c} T^{b}\right) \vec{I} \cdot \frac{z_{2} \vec{p}_{2}-\left(1-z_{2}\right)\left(\vec{k}_{g}-\vec{q}_{13}\right)}{\left(z_{2} \vec{p}_{2}-\left(1-z_{2}\right)\left(\vec{k}_{g}-\vec{q}_{13}\right)\right)^{2}}$

$$
\times \Psi_{q q q}^{*}\left(x_{1}-x_{g}, \vec{k}_{1}+x_{1} \vec{q}-\vec{q}_{2}-\vec{k}_{g}+x_{g} \vec{K} ; x_{2}+x_{g}, \vec{k}_{2}+x_{2} \vec{q}-\vec{q}_{13}+\vec{k}_{g}-x_{g} \vec{K} ; x_{3}, \vec{k}_{3}+x_{3} \vec{q}\right) .
$$

The symmetry factor of 6 includes a factor of 2 due to the contribution of diagram Fig.4 $\left(g q_{2} g\right)$ (not shown) with the gluon emission and absorption vertices between quarks 1 and 2 interchanged.

$$
\begin{aligned}
\text { Fig.4(ggq })= & -\frac{1}{4} \operatorname{tr}\left(T^{b} T^{c} D^{a}-T^{a} T^{b} T^{c}\right) \vec{I} \cdot \frac{z_{2} \vec{p}_{2}-\left(1-z_{2}\right)\left(\vec{k}_{g}-\vec{q}_{23}\right)}{\left(z_{2} \vec{p}_{2}-\left(1-z_{2}\right)\left(\vec{k}_{g}-\vec{q}_{23}\right)\right)^{2}} \\
& \times \Psi_{q q q}^{*}\left(x_{1}-x_{g}, \vec{k}_{1}+x_{1} \vec{q}-\vec{q}_{1}-\vec{k}_{g}+x_{g} \vec{K} ; x_{2}+x_{g}, \vec{k}_{2}+x_{2} \vec{q}-\vec{q}_{23}+\vec{k}_{g}-x_{g} \vec{K} ; x_{3}, \vec{k}_{3}+x_{3} \vec{q}\right) .
\end{aligned}
$$

Again, the symmetry factor for this diagram is 6 which includes the contribution of diagram Fig.4( $\left.g g q_{2}\right)$ (not shown) with the gluon emission and absorption vertices between quarks 1 and 2 interchanged,

$$
\begin{aligned}
\text { Fig.4(q } \left.q_{2} g g\right)= & -\frac{1}{4} \operatorname{tr}\left(T^{a} T^{b} D^{c}-T^{a} T^{b} T^{c}\right) \vec{I} \cdot \frac{z_{2}\left(\vec{p}_{2}-\vec{q}_{3}\right)-\left(1-z_{2}\right)\left(\vec{k}_{g}-\vec{q}_{12}\right)}{\left(z_{2}\left(\vec{p}_{2}-\vec{q}_{3}\right)-\left(1-z_{2}\right)\left(\vec{k}_{g}-\vec{q}_{12}\right)\right)^{2}} \\
& \times \Psi_{q q q}^{*}\left(x_{1}-x_{g}, \vec{k}_{1}+x_{1} \vec{q}-\vec{k}_{g}+x_{g} \vec{K} ; x_{2}+x_{g}, \vec{k}_{2}+x_{2} \vec{q}-\vec{q}+\vec{k}_{g}-x_{g} \vec{K} ; x_{3}, \vec{k}_{3}+x_{3} \vec{q}\right) .
\end{aligned}
$$

The symmetry factor is 6; this includes the contribution from Fig.4 $\left(q_{1} g g\right)$ with the gluon emission and absorption vertices between quarks 1 and 2 interchanged,

$$
\begin{aligned}
\text { Fig.4(gq } 2 g)= & -\frac{1}{4} \operatorname{tr}\left(T^{a} T^{c} D^{b}-T^{a} T^{c} T^{b}\right) \vec{I} \cdot \frac{z_{2}\left(\vec{p}_{2}-\vec{q}_{2}\right)-\left(1-z_{2}\right)\left(\vec{k}_{g}-\vec{q}_{13}\right)}{\left(z_{2}\left(\vec{p}_{2}-\vec{q}_{2}\right)-\left(1-z_{2}\right)\left(\vec{k}_{g}-\vec{q}_{13}\right)\right)^{2}} \\
& \times \Psi_{q q q}^{*}\left(x_{1}-x_{g}, \vec{k}_{1}+x_{1} \vec{q}-\vec{k}_{g}+x_{g} \vec{K} ; x_{2}+x_{g}, \vec{k}_{2}+x_{2} \vec{q}-\vec{q}+\vec{k}_{g}-x_{g} \vec{K} ; x_{3}, \vec{k}_{3}+x_{3} \vec{q}\right) .
\end{aligned}
$$

The symmetry factor is 6 .

$$
\begin{aligned}
& \text { Fig.4 }\left(g g q_{2}\right)=-\frac{1}{4} \operatorname{tr}\left(T^{a} T^{b} D^{c}-T^{a} T^{b} T^{c}\right) \vec{I} \cdot \frac{z_{2}\left(\vec{p}_{2}-\vec{q}_{1}\right)-\left(1-z_{2}\right)\left(\vec{k}_{g}-\vec{q}_{23}\right)}{\left(z_{2}\left(\vec{p}_{2}-\vec{q}_{1}\right)-\left(1-z_{2}\right)\left(\vec{k}_{g}-\vec{q}_{23}\right)\right)^{2}} \\
& \times \Psi_{q q q}^{*}\left(x_{1}-x_{g}, \vec{k}_{1}+x_{1} \vec{q}-\vec{k}_{g}+x_{g} \vec{K} ; x_{2}+x_{g}, \vec{k}_{2}+x_{2} \vec{q}-\vec{q}+\vec{k}_{g}-x_{g} \vec{K} ; x_{3}, \vec{k}_{3}+x_{3} \vec{q}\right) \text {. }
\end{aligned}
$$

The symmetry factor is 6 .

$$
\begin{aligned}
\text { Fig.4(q } \left.q_{3} g g\right)= & \frac{1}{2} \operatorname{tr} T^{a} T^{b} D^{c} \vec{I} \cdot \frac{z_{2} \vec{p}_{2}-\left(1-z_{2}\right)\left(\vec{k}_{g}-\vec{q}_{12}\right)}{\left(z_{2} \vec{p}_{2}-\left(1-z_{2}\right)\left(\vec{k}_{g}-\vec{q}_{12}\right)\right)^{2}} \\
& \times \Psi_{q q q}^{*}\left(x_{1}-x_{g}, \vec{k}_{1}+x_{1} \vec{q}-\vec{k}_{g}+x_{g} \vec{K}^{\prime} x_{2}+x_{g}, \vec{k}_{2}+x_{2} \vec{q}-\vec{q}_{12}+\vec{k}_{g}-x_{g} \vec{K} ; x_{3}, \vec{k}_{3}+x_{3} \vec{q}-\vec{q}_{3}\right) .
\end{aligned}
$$

The symmetry factor is 6 .

$$
\begin{aligned}
\text { Fig.4(gq } 3 g)= & \frac{1}{2} \operatorname{tr} T^{a} T^{c} D^{b} \vec{I} \cdot \frac{z_{2} \vec{p}_{2}-\left(1-z_{2}\right)\left(\vec{k}_{g}-\vec{q}_{13}\right)}{\left(z_{2} \vec{p}_{2}-\left(1-z_{2}\right)\left(\vec{k}_{g}-\vec{q}_{13}\right)\right)^{2}} \\
& \times \Psi_{q q q}^{*}\left(x_{1}-x_{g}, \vec{k}_{1}+x_{1} \vec{q}-\vec{k}_{g}+x_{g} \vec{K} ; x_{2}+x_{g}, \vec{k}_{2}+x_{2} \vec{q}-\vec{q}_{13}+\vec{k}_{g}-x_{g} \vec{K} ; x_{3}, \vec{k}_{3}+x_{3} \vec{q}-\vec{q}_{2}\right) .
\end{aligned}
$$

The symmetry factor is 6 . 


$$
\begin{aligned}
& \text { Fig. } 4\left(g g q_{3}\right)=\frac{1}{2} \operatorname{tr} T^{a} T^{b} D^{c} \vec{I} \cdot \frac{z_{2} \vec{p}_{2}-\left(1-z_{2}\right)\left(\vec{k}_{g}-\vec{q}_{23}\right)}{\left(z_{2} \vec{p}_{2}-\left(1-z_{2}\right)\left(\vec{k}_{g}-\vec{q}_{23}\right)\right)^{2}} \\
& \times \Psi_{q q q}^{*}\left(x_{1}-x_{g}, \vec{k}_{1}+x_{1} \vec{q}-\vec{k}_{g}+x_{g} \vec{K} ; x_{2}+x_{g}, \vec{k}_{2}+x_{2} \vec{q}-\vec{q}_{23}+\vec{k}_{g}-x_{g} \vec{K} ; x_{3}, \vec{k}_{3}+x_{3} \vec{q}-\vec{q}_{1}\right) .
\end{aligned}
$$

The symmetry factor is 6 .

We continue with the diagrams shown in Fig. 5 where two distinct quarks exchange a gluon across the operator insertion, and where one of the probes attaches to that gluon,

$$
\begin{aligned}
\text { Fig.5 }\left(q_{1} q_{1} g\right)= & \frac{N_{c}}{2} \operatorname{tr} t^{a} t^{b} t^{c} \vec{I} \cdot \frac{z_{2} \vec{p}_{2}-\left(1-z_{2}\right)\left(\vec{k}_{g}-\vec{q}_{1}\right)}{\left(z_{2} \vec{p}_{2}-\left(1-z_{2}\right)\left(\vec{k}_{g}-\vec{q}_{1}\right)\right)^{2}} \\
& \times \Psi_{q q q}^{*}\left(x_{1}-x_{g}, \vec{k}_{1}+x_{1} \vec{q}-\vec{k}_{g}+x_{g} \vec{K}-\vec{q}_{23} ; x_{2}+x_{g}, \vec{k}_{2}+x_{2} \vec{q}-\vec{q}_{1}+\vec{k}_{g}-x_{g} \vec{K} ; x_{3}, \vec{k}_{3}+x_{3} \vec{q}\right) .
\end{aligned}
$$

The symmetry factor is 6 [this includes the contribution from diagram Fig.5 $\left(q_{2} q_{2} g\right)$ (not shown) with the gluon emission and absorption vertices between quarks 1 and 2 interchanged],

$$
\begin{aligned}
\text { Fig.5 }\left(q_{1} g q_{1}\right)= & \frac{N_{c}}{2} \operatorname{trt} t^{a} t^{c} t^{b} \vec{I} \cdot \frac{z_{2} \vec{p}_{2}-\left(1-z_{2}\right)\left(\vec{k}_{g}-\vec{q}_{2}\right)}{\left(z_{2} \vec{p}_{2}-\left(1-z_{2}\right)\left(\vec{k}_{g}-\vec{q}_{2}\right)\right)^{2}} \\
& \times \Psi_{q q q}^{*}\left(x_{1}-x_{g}, \vec{k}_{1}+x_{1} \vec{q}-\vec{k}_{g}+x_{g} \vec{K}-\vec{q}_{13} ; x_{2}+x_{g}, \vec{k}_{2}+x_{2} \vec{q}-\vec{q}_{2}+\vec{k}_{g}-x_{g} \vec{K} ; x_{3}, \vec{k}_{3}+x_{3} \vec{q}\right) .
\end{aligned}
$$

Again, the symmetry factor is 6 [this includes the contribution from diagram Fig.5 $\left(q_{2} g q_{2}\right)$ (not shown) with the gluon emission and absorption vertices between quarks 1 and 2 interchanged],

$$
\begin{aligned}
\text { Fig.5 }\left(g q_{1} q_{1}\right)= & \frac{N_{c}}{2} \operatorname{tr} t^{a} t^{b} t^{c} \vec{I} \cdot \frac{z_{2} \vec{p}_{2}-\left(1-z_{2}\right)\left(\vec{k}_{g}-\vec{q}_{3}\right)}{\left(z_{2} \vec{p}_{2}-\left(1-z_{2}\right)\left(\vec{k}_{g}-\vec{q}_{3}\right)\right)^{2}} \\
& \times \Psi_{q q q}^{*}\left(x_{1}-x_{g}, \vec{k}_{1}+x_{1} \vec{q}-\vec{k}_{g}+x_{g} \vec{K}-\vec{q}_{12} ; x_{2}+x_{g}, \vec{k}_{2}+x_{2} \vec{q}-\vec{q}_{3}+\vec{k}_{g}-x_{g} \vec{K} ; x_{3}, \vec{k}_{3}+x_{3} \vec{q}\right) .
\end{aligned}
$$

Again, the symmetry factor is 6 [this includes the contribution from diagram Fig.5 $\left(g q_{2} q_{2}\right)$ (not shown) with the gluon emission and absorption vertices between quarks 1 and 2 interchanged],

$$
\begin{aligned}
\text { Fig.5 } 5\left(q_{2} q_{1} g\right)= & \left(-\frac{1}{4}\left(T^{a}\right)_{b c}+\frac{N_{c}}{2} \operatorname{tr} t^{a} t^{c} t^{b}\right) \vec{I} \cdot \frac{z_{2}\left(\vec{p}_{2}-\vec{q}_{3}\right)-\left(1-z_{2}\right)\left(\vec{k}_{g}-\vec{q}_{1}\right)}{\left(z_{2}\left(\vec{p}_{2}-\vec{q}_{3}\right)-\left(1-z_{2}\right)\left(\vec{k}_{g}-\vec{q}_{1}\right)\right)^{2}} \\
& \times \Psi_{q q q}^{*}\left(x_{1}-x_{g}, \vec{k}_{1}+x_{1} \vec{q}-\vec{k}_{g}+x_{g} \vec{K}-\vec{q}_{2} ; x_{2}+x_{g}, \vec{k}_{2}+x_{2} \vec{q}-\vec{q}_{13}+\vec{k}_{g}-x_{g} \vec{K} ; x_{3}, \vec{k}_{3}+x_{3} \vec{q}\right) .
\end{aligned}
$$

The symmetry factor is 6, including the contribution from diagram Fig.5 $\left(q_{1} q_{2} g\right)$ (not shown) with the gluon emission and absorption vertices between quarks 1 and 2 interchanged,

$$
\begin{aligned}
\text { Fig.5 }\left(q_{2} g q_{1}\right)= & \left(\frac{1}{4}\left(T^{b}\right)_{c a}+\frac{N_{c}}{2} \operatorname{tr} t^{a} t^{b} t^{c}\right) \vec{I} \cdot \frac{z_{2}\left(\vec{p}_{2}-\vec{q}_{3}\right)-\left(1-z_{2}\right)\left(\vec{k}_{g}-\vec{q}_{2}\right)}{\left(z_{2}\left(\vec{p}_{2}-\vec{q}_{3}\right)-\left(1-z_{2}\right)\left(\vec{k}_{g}-\vec{q}_{2}\right)\right)^{2}} \\
& \times \Psi_{q q q}^{*}\left(x_{1}-x_{g}, \vec{k}_{1}+x_{1} \vec{q}-\vec{k}_{g}+x_{g} \vec{K}-\vec{q}_{1} ; x_{2}+x_{g}, \vec{k}_{2}+x_{2} \vec{q}-\vec{q}_{23}+\vec{k}_{g}-x_{g} \vec{K} ; x_{3}, \vec{k}_{3}+x_{3} \vec{q}\right) .
\end{aligned}
$$

The symmetry factor is 6, including the contribution from diagram Fig.5 $\left(q_{1} g q_{2}\right)$ (not shown) with the gluon emission and absorption vertices between quarks 1 and 2 interchanged,

$$
\begin{aligned}
\text { Fig.5 }\left(g q_{2} q_{1}\right)= & \left(\frac{1}{4}\left(T^{c}\right)_{b a}+\frac{N_{c}}{2} \operatorname{tr} t^{a} t^{c} t^{b}\right) \vec{I} \cdot \frac{z_{2}\left(\vec{p}_{2}-\vec{q}_{2}\right)-\left(1-z_{2}\right)\left(\vec{k}_{g}-\vec{q}_{3}\right)}{\left(z_{2}\left(\vec{p}_{2}-\vec{q}_{2}\right)-\left(1-z_{2}\right)\left(\vec{k}_{g}-\vec{q}_{3}\right)\right)^{2}} \\
& \times \Psi_{q q q}^{*}\left(x_{1}-x_{g}, \vec{k}_{1}+x_{1} \vec{q}-\vec{k}_{g}+x_{g} \vec{K}-\vec{q}_{1} ; x_{2}+x_{g}, \vec{k}_{2}+x_{2} \vec{q}-\vec{q}_{23}+\vec{k}_{g}-x_{g} \vec{K} ; x_{3}, \vec{k}_{3}+x_{3} \vec{q}\right) .
\end{aligned}
$$


The symmetry factor is 6 , including the contribution from diagram Fig.5 $\left(g q_{1} q_{2}\right)$ (not shown) with the gluon emission and absorption vertices between quarks 1 and 2 interchanged,

$$
\begin{aligned}
\text { Fig. } 5\left(q_{1} q_{2} g\right)= & \left(\frac{1}{4}\left(T^{a}\right)_{b c}+\frac{N_{c}}{2} \operatorname{tr} t^{a} t^{b} t^{c}\right) \vec{I} \cdot \frac{z_{2}\left(\vec{p}_{2}-\vec{q}_{2}\right)-\left(1-z_{2}\right)\left(\vec{k}_{g}-\vec{q}_{1}\right)}{\left(z_{2}\left(\vec{p}_{2}-\vec{q}_{2}\right)-\left(1-z_{2}\right)\left(\vec{k}_{g}-\vec{q}_{1}\right)\right)^{2}} \\
& \times \Psi_{q q q}^{*}\left(x_{1}-x_{g}, \vec{k}_{1}+x_{1} \vec{q}-\vec{k}_{g}+x_{g} \vec{K}-\vec{q}_{3} ; x_{2}+x_{g}, \vec{k}_{2}+x_{2} \vec{q}-\vec{q}_{12}+\vec{k}_{g}-x_{g} \vec{K} ; x_{3}, \vec{k}_{3}+x_{3} \vec{q}\right) .
\end{aligned}
$$

The symmetry factor is 6 , including the contribution from diagram Fig. $5\left(q_{2} q_{1} g\right)$ with the gluon emission and absorption vertices between quarks 1 and 2 interchanged,

$$
\begin{aligned}
\text { Fig.5 }\left(q_{1} g q_{2}\right)= & \left(\frac{1}{4}\left(T^{b}\right)_{a c}+\frac{N_{c}}{2} \operatorname{tr} t^{a} t^{c} t^{b}\right) \vec{I} \cdot \frac{z_{2}\left(\vec{p}_{2}-\vec{q}_{1}\right)-\left(1-z_{2}\right)\left(\vec{k}_{g}-\vec{q}_{2}\right)}{\left(z_{2}\left(\vec{p}_{2}-\vec{q}_{1}\right)-\left(1-z_{2}\right)\left(\vec{k}_{g}-\vec{q}_{2}\right)\right)^{2}} \\
& \times \Psi_{q q q}^{*}\left(x_{1}-x_{g}, \vec{k}_{1}+x_{1} \vec{q}-\vec{k}_{g}+x_{g} \vec{K}-\vec{q}_{3} ; x_{2}+x_{g}, \vec{k}_{2}+x_{2} \vec{q}-\vec{q}_{12}+\vec{k}_{g}-x_{g} \vec{K} ; x_{3}, \vec{k}_{3}+x_{3} \vec{q}\right) .
\end{aligned}
$$

The symmetry factor is 6 , including the contribution from diagram Fig. $5\left(q_{2} g q_{1}\right)$ with the gluon emission and absorption vertices between quarks 1 and 2 interchanged,

$$
\begin{aligned}
\text { Fig.5 }\left(g q_{1} q_{2}\right)= & \left(\frac{1}{4}\left(T^{c}\right)_{a b}+\frac{N_{c}}{2} \operatorname{tr} t^{a} t^{b} t^{c}\right) \vec{I} \cdot \frac{z_{2}\left(\vec{p}_{2}-\vec{q}_{1}\right)-\left(1-z_{2}\right)\left(\vec{k}_{g}-\vec{q}_{3}\right)}{\left(z_{2}\left(\vec{p}_{2}-\vec{q}_{1}\right)-\left(1-z_{2}\right)\left(\vec{k}_{g}-\vec{q}_{3}\right)\right)^{2}} \\
& \times \Psi_{q q q}^{*}\left(x_{1}-x_{g}, \vec{k}_{1}+x_{1} \vec{q}-\vec{k}_{g}+x_{g} \vec{K}-\vec{q}_{2} ; x_{2}+x_{g}, \vec{k}_{2}+x_{2} \vec{q}-\vec{q}_{13}+\vec{k}_{g}-x_{g} \vec{K} ; x_{3}, \vec{k}_{3}+x_{3} \vec{q}\right) .
\end{aligned}
$$

The symmetry factor is 6 , including the contribution from diagram Fig. $5\left(g q_{2} q_{1}\right)$ with the gluon emission and absorption vertices between quarks 1 and 2 interchanged,

$$
\begin{aligned}
\text { Fig.5 }\left(q_{2} q_{2} g\right)= & \frac{N_{c}}{2} \operatorname{tr} t^{a} t^{b} t^{c} \vec{I} \cdot \frac{z_{2}\left(\vec{p}_{2}-\vec{q}_{23}\right)-\left(1-z_{2}\right)\left(\vec{k}_{g}-\vec{q}_{1}\right)}{\left(z_{2}\left(\vec{p}_{2}-\vec{q}_{23}\right)-\left(1-z_{2}\right)\left(\vec{k}_{g}-\vec{q}_{1}\right)\right)^{2}} \\
& \times \Psi_{q q q}^{*}\left(x_{1}-x_{g}, \vec{k}_{1}+x_{1} \vec{q}-\vec{k}_{g}+x_{g} \vec{K} ; x_{2}+x_{g}, \vec{k}_{2}+x_{2} \vec{q}-\vec{q}+\vec{k}_{g}-x_{g} \vec{K} ; x_{3}, \vec{k}_{3}+x_{3} \vec{q}\right) .
\end{aligned}
$$

The symmetry factor is 6 , including the contribution from diagram Fig. $5\left(q_{1} q_{1} g\right)$ with the gluon emission and absorption vertices between quarks 1 and 2 interchanged,

$$
\begin{aligned}
\text { Fig.5 }\left(q_{2} g q_{2}\right)= & \frac{N_{c}}{2} \operatorname{trt} t^{b} t^{a} t^{c} \vec{I} \cdot \frac{z_{2}\left(\vec{p}_{2}-\vec{q}_{13}\right)-\left(1-z_{2}\right)\left(\vec{k}_{g}-\vec{q}_{2}\right)}{\left(z_{2}\left(\vec{p}_{2}-\vec{q}_{13}\right)-\left(1-z_{2}\right)\left(\vec{k}_{g}-\vec{q}_{2}\right)\right)^{2}} \\
& \times \Psi_{q q q}^{*}\left(x_{1}-x_{g}, \vec{k}_{1}+x_{1} \vec{q}-\vec{k}_{g}+x_{g} \vec{K} ; x_{2}+x_{g}, \vec{k}_{2}+x_{2} \vec{q}-\vec{q}+\vec{k}_{g}-x_{g} \vec{K} ; x_{3}, \vec{k}_{3}+x_{3} \vec{q}\right) .
\end{aligned}
$$

The symmetry factor is 6 , including the contribution from diagram Fig.5 $\left(q_{1} g q_{1}\right)$ with the gluon emission and absorption vertices between quarks 1 and 2 interchanged,

$$
\begin{aligned}
\text { Fig.5 }\left(g q_{2} q_{2}\right)= & \frac{N_{c}}{2} \operatorname{tr} t^{a} t^{b} t^{c} \vec{I} \cdot \frac{z_{2}\left(\vec{p}_{2}-\vec{q}_{12}\right)-\left(1-z_{2}\right)\left(\vec{k}_{g}-\vec{q}_{3}\right)}{\left(z_{2}\left(\vec{p}_{2}-\vec{q}_{12}\right)-\left(1-z_{2}\right)\left(\vec{k}_{g}-\vec{q}_{3}\right)\right)^{2}} \\
& \times \Psi_{q q q}^{*}\left(x_{1}-x_{g}, \vec{k}_{1}+x_{1} \vec{q}-\vec{k}_{g}+x_{g} \vec{K} ; x_{2}+x_{g}, \vec{k}_{2}+x_{2} \vec{q}-\vec{q}+\vec{k}_{g}-x_{g} \vec{K} ; x_{3}, \vec{k}_{3}+x_{3} \vec{q}\right) .
\end{aligned}
$$

The symmetry factor is 6 , including the contribution from diagram Fig.5 $\left(g q_{1} q_{1}\right)$ with the gluon emission and absorption vertices between quarks 1 and 2 interchanged, 
Fig. $5\left(q_{3} q_{1} g\right)=\left(-\frac{1}{4}\left(T^{a}\right)_{c b}-N_{c} \operatorname{tr} t^{a} t^{c} t^{b}+\frac{1}{4} \operatorname{tr} T^{c} T^{a}\left(D^{b}-T^{b}\right)\right) \vec{I} \cdot \frac{z_{2} \vec{p}_{2}-\left(1-z_{2}\right)\left(\vec{k}_{g}-\vec{q}_{1}\right)}{\left(z_{2} \vec{p}_{2}-\left(1-z_{2}\right)\left(\vec{k}_{g}-\vec{q}_{1}\right)\right)^{2}}$

$$
\times \Psi_{q q q}^{*}\left(x_{1}-x_{g}, \vec{k}_{1}+x_{1} \vec{q}-\vec{k}_{g}+x_{g} \vec{K}-\vec{q}_{2} ; x_{2}+x_{g}, \vec{k}_{2}+x_{2} \vec{q}-\vec{q}_{1}+\vec{k}_{g}-x_{g} \vec{K} ; x_{3}, \vec{k}_{3}+x_{3} \vec{q}-\vec{q}_{3}\right) .
$$

The symmetry factor of 6 includes the contribution from diagram Fig.5 $\left(q_{3} q_{2} g\right)$ with the gluon emission and absorption vertices between quarks 1 and 2 interchanged,

Fig. $5\left(q_{3} g q_{1}\right)=\left(-\frac{1}{4}\left(T^{b}\right)_{c a}-N_{c} \operatorname{tr} t^{a} t^{b} t^{c}+\frac{1}{4} \operatorname{tr} T^{c} T^{b}\left(D^{a}-T^{a}\right)\right) \vec{I} \cdot \frac{z_{2} \vec{p}_{2}-\left(1-z_{2}\right)\left(\vec{k}_{g}-\vec{q}_{2}\right)}{\left(z_{2} \vec{p}_{2}-\left(1-z_{2}\right)\left(\vec{k}_{g}-\vec{q}_{2}\right)\right)^{2}}$

$$
\times \Psi_{q q q}^{*}\left(x_{1}-x_{g}, \vec{k}_{1}+x_{1} \vec{q}-\vec{k}_{g}+x_{g} \vec{K}-\vec{q}_{1} ; x_{2}+x_{g}, \vec{k}_{2}+x_{2} \vec{q}-\vec{q}_{2}+\vec{k}_{g}-x_{g} \vec{K} ; x_{3}, \vec{k}_{3}+x_{3} \vec{q}-\vec{q}_{3}\right) .
$$

The symmetry factor of 6 includes the contribution from diagram Fig.5 $\left(q_{3} g q_{2}\right)$ with the gluon emission and absorption vertices between quarks 1 and 2 interchanged,

Fig. $5\left(g q_{3} q_{1}\right)=\left(-\frac{1}{4}\left(T^{c}\right)_{b a}-N_{c} \operatorname{tr} t^{a} t^{c} t^{b}+\frac{1}{4} \operatorname{tr} T^{b} T^{c}\left(D^{a}-T^{a}\right)\right) \vec{I} \cdot \frac{z_{2} \vec{p}_{2}-\left(1-z_{2}\right)\left(\vec{k}_{g}-\vec{q}_{3}\right)}{\left(z_{2} \vec{p}_{2}-\left(1-z_{2}\right)\left(\vec{k}_{g}-\vec{q}_{3}\right)\right)^{2}}$

$$
\times \Psi_{q q q}^{*}\left(x_{1}-x_{g}, \vec{k}_{1}+x_{1} \vec{q}-\vec{k}_{g}+x_{g} \vec{K}-\vec{q}_{1} ; x_{2}+x_{g}, \vec{k}_{2}+x_{2} \vec{q}-\vec{q}_{3}+\vec{k}_{g}-x_{g} \vec{K} ; x_{3}, \vec{k}_{3}+x_{3} \vec{q}-\vec{q}_{2}\right) .
$$

The symmetry factor of 6 includes the contribution from diagram Fig.5 $\left(g q_{3} q_{2}\right)$ with the gluon emission and absorption vertices between quarks 1 and 2 interchanged,

Fig. $5\left(q_{1} q_{3} g\right)=\left(-\frac{1}{4}\left(T^{a}\right)_{b c}-N_{c} \operatorname{tr} t^{a} t^{b} t^{c}+\frac{1}{4} \operatorname{tr} T^{b} T^{a}\left(D^{c}-T^{c}\right)\right) \vec{I} \cdot \frac{z_{2} \vec{p}_{2}-\left(1-z_{2}\right)\left(\vec{k}_{g}-\vec{q}_{1}\right)}{\left(z_{2} \vec{p}_{2}-\left(1-z_{2}\right)\left(\vec{k}_{g}-\vec{q}_{1}\right)\right)^{2}}$

$$
\times \Psi_{q q q}^{*}\left(x_{1}-x_{g}, \vec{k}_{1}+x_{1} \vec{q}-\vec{k}_{g}+x_{g} \vec{K}-\vec{q}_{3} ; x_{2}+x_{g}, \vec{k}_{2}+x_{2} \vec{q}-\vec{q}_{1}+\vec{k}_{g}-x_{g} \vec{K} ; x_{3}, \vec{k}_{3}+x_{3} \vec{q}-\vec{q}_{2}\right) .
$$

The symmetry factor of 6 includes the contribution from diagram Fig.5 $\left(q_{2} q_{3} g\right)$ with the gluon emission and absorption vertices between quarks 1 and 2 interchanged.

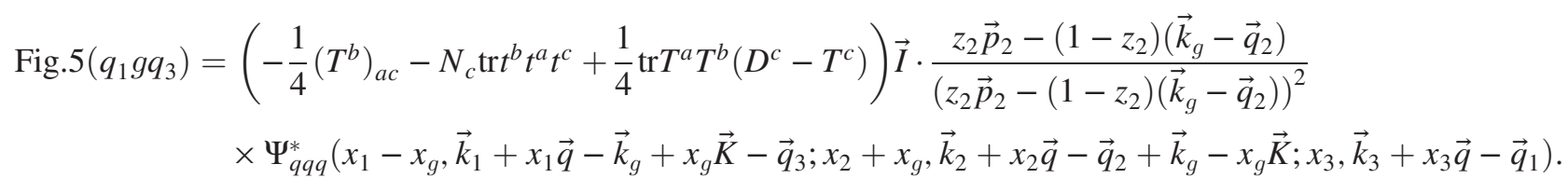

The symmetry factor of 6 includes the contribution from diagram Fig.5 $\left(q_{2} g q_{3}\right)$ with the gluon emission and absorption vertices between quarks 1 and 2 interchanged,

$\begin{aligned} \text { Fig.5 }\left(g q_{1} q_{3}\right)= & \left(-\frac{1}{4}\left(T^{c}\right)_{a b}-N_{c} \operatorname{tr} t^{a} t^{b} t^{c}+\frac{1}{4} \operatorname{tr} T^{a} T^{c}\left(D^{b}-T^{b}\right)\right) \vec{I} \cdot \frac{z_{2} \vec{p}_{2}-\left(1-z_{2}\right)\left(\vec{k}_{g}-\vec{q}_{3}\right)}{\left(z_{2} \vec{p}_{2}-\left(1-z_{2}\right)\left(\vec{k}_{g}-\vec{q}_{3}\right)\right)^{2}} \\ & \times \Psi_{q q q}^{*}\left(x_{1}-x_{g}, \vec{k}_{1}+x_{1} \vec{q}-\vec{k}_{g}+x_{g} \vec{K}-\vec{q}_{2} ; x_{2}+x_{g}, \vec{k}_{2}+x_{2} \vec{q}-\vec{q}_{3}+\vec{k}_{g}-x_{g} \vec{K} ; x_{3}, \vec{k}_{3}+x_{3} \vec{q}-\vec{q}_{1}\right) .\end{aligned}$

The symmetry factor of 6 includes the contribution from diagram Fig.5 $\left(g q_{2} q_{3}\right)$ with the gluon emission and absorption vertices between quarks 1 and 2 interchanged,

$$
\begin{aligned}
\text { Fig.5 }\left(q_{3} q_{2} g\right)= & \left(-\frac{1}{4}\left(T^{a}\right)_{b c}-\frac{N_{c}}{4}\left(D^{a}\right)_{b c}+\frac{1}{4} \operatorname{tr} T^{a}\left(D^{b}-T^{b}\right) T^{c}\right) \vec{I} \cdot \frac{z_{2}\left(\vec{p}_{2}-\vec{q}_{2}\right)-\left(1-z_{2}\right)\left(\vec{k}_{g}-\vec{q}_{1}\right)}{\left(z_{2}\left(\vec{p}_{2}-\vec{q}_{2}\right)-\left(1-z_{2}\right)\left(\vec{k}_{g}-\vec{q}_{1}\right)\right)^{2}} \\
& \times \Psi_{q q q}^{*}\left(x_{1}-x_{g}, \vec{k}_{1}+x_{1} \vec{q}-\vec{k}_{g}+x_{g} \vec{K} ; x_{2}+x_{g}, \vec{k}_{2}+x_{2} \vec{q}-\vec{q}_{12}+\vec{k}_{g}-x_{g} \vec{K} ; x_{3}, \vec{k}_{3}+x_{3} \vec{q}-\vec{q}_{3}\right) .
\end{aligned}
$$

The symmetry factor of 6 includes the contribution from diagram Fig.5 $\left(q_{3} q_{1} g\right)$ with the gluon emission and absorption vertices between quarks 1 and 2 interchanged, 

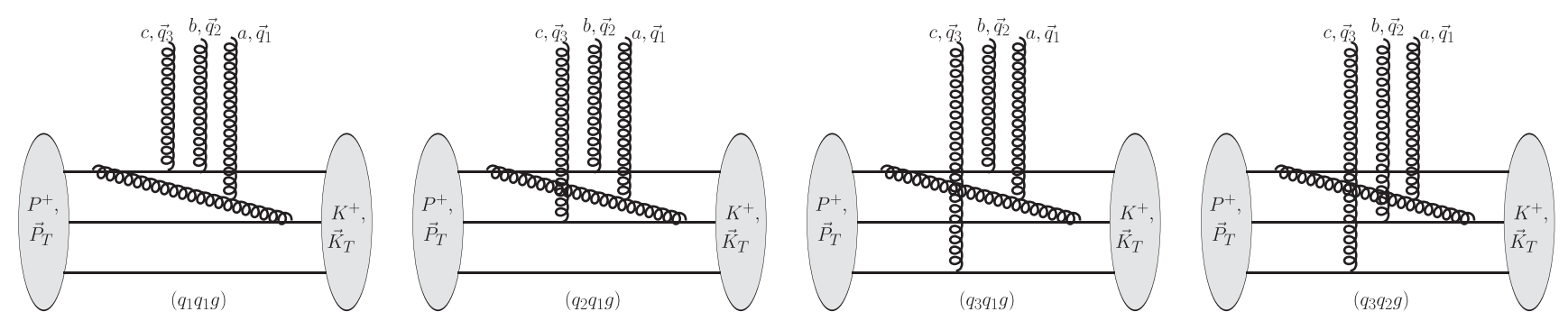

FIG. 5. A sample of UV finite diagrams for $\left\langle\rho^{a}\left(\vec{q}_{1}\right) \rho^{b}\left(\vec{q}_{2}\right) \rho^{c}\left(\vec{q}_{3}\right)\right\rangle$ where one of the probes attaches to the gluon in the proton. The cut is located at the insertion of the three color charge operators.

$$
\begin{aligned}
\text { Fig. } 5\left(q_{3} g q_{2}\right)= & \left(-\frac{1}{4}\left(T^{b}\right)_{a c}-\frac{N_{c}}{4}\left(D^{b}\right)_{a c}+\frac{1}{4} \operatorname{tr} T^{b}\left(D^{a}-T^{a}\right) T^{c}\right) \vec{I} \cdot \frac{z_{2}\left(\vec{p}_{2}-\vec{q}_{1}\right)-\left(1-z_{2}\right)\left(\vec{k}_{g}-\vec{q}_{2}\right)}{\left(z_{2}\left(\vec{p}_{2}-\vec{q}_{1}\right)-\left(1-z_{2}\right)\left(\vec{k}_{g}-\vec{q}_{2}\right)\right)^{2}} \\
& \times \Psi_{q q q}^{*}\left(x_{1}-x_{g}, \vec{k}_{1}+x_{1} \vec{q}-\vec{k}_{g}+x_{g} \vec{K} ; x_{2}+x_{g}, \vec{k}_{2}+x_{2} \vec{q}-\vec{q}_{12}+\vec{k}_{g}-x_{g} \vec{K} ; x_{3}, \vec{k}_{3}+x_{3} \vec{q}-\vec{q}_{3}\right) .
\end{aligned}
$$

The symmetry factor of 6 includes the contribution from diagram Fig.5 $\left(q_{3} g q_{1}\right)$ with the gluon emission and absorption vertices between quarks 1 and 2 interchanged,

$$
\begin{aligned}
\text { Fig. } 5\left(g q_{3} q_{2}\right)= & \left(-\frac{1}{4}\left(T^{c}\right)_{a b}-\frac{N_{c}}{4}\left(D^{c}\right)_{a b}+\frac{1}{4} \operatorname{tr} T^{c}\left(D^{a}-T^{a}\right) T^{b}\right) \vec{I} \cdot \frac{z_{2}\left(\vec{p}_{2}-\vec{q}_{1}\right)-\left(1-z_{2}\right)\left(\vec{k}_{g}-\vec{q}_{3}\right)}{\left(z_{2}\left(\vec{p}_{2}-\vec{q}_{1}\right)-\left(1-z_{2}\right)\left(\vec{k}_{g}-\vec{q}_{3}\right)\right)^{2}} \\
& \times \Psi_{q q q}^{*}\left(x_{1}-x_{g}, \vec{k}_{1}+x_{1} \vec{q}-\vec{k}_{g}+x_{g} \vec{K} ; x_{2}+x_{g}, \vec{k}_{2}+x_{2} \vec{q}-\vec{q}_{13}+\vec{k}_{g}-x_{g} \vec{K} ; x_{3}, \vec{k}_{3}+x_{3} \vec{q}-\vec{q}_{2}\right) .
\end{aligned}
$$

The symmetry factor of 6 includes the contribution from diagram Fig.5 $\left(g q_{3} q_{1}\right)$ with the gluon emission and absorption vertices between quarks 1 and 2 interchanged,

$$
\begin{aligned}
\text { Fig.5 }\left(q_{2} q_{3} g\right)= & \left(\frac{1}{4}\left(T^{a}\right)_{b c}-\frac{N_{c}}{4}\left(D^{a}\right)_{b c}+\frac{1}{4} \operatorname{tr} T^{a}\left(D^{c}-T^{c}\right) T^{b}\right) \vec{I} \cdot \frac{z_{2}\left(\vec{p}_{2}-\vec{q}_{3}\right)-\left(1-z_{2}\right)\left(\vec{k}_{g}-\vec{q}_{1}\right)}{\left(z_{2}\left(\vec{p}_{2}-\vec{q}_{3}\right)-\left(1-z_{2}\right)\left(\vec{k}_{g}-\vec{q}_{1}\right)\right)^{2}} \\
& \times \Psi_{q q q}^{*}\left(x_{1}-x_{g}, \vec{k}_{1}+x_{1} \vec{q}-\vec{k}_{g}+x_{g} \vec{K} ; x_{2}+x_{g}, \vec{k}_{2}+x_{2} \vec{q}-\vec{q}_{13}+\vec{k}_{g}-x_{g} \vec{K} ; x_{3}, \vec{k}_{3}+x_{3} \vec{q}-\vec{q}_{2}\right) .
\end{aligned}
$$

The symmetry factor of 6 includes the contribution from diagram Fig.5 $\left(q_{1} q_{3} g\right)$ with the gluon emission and absorption vertices between quarks 1 and 2 interchanged,

$$
\begin{aligned}
\text { Fig.5 }\left(q_{2} g q_{3}\right)= & \left(\frac{1}{4}\left(T^{b}\right)_{a c}-\frac{N_{c}}{4}\left(D^{b}\right)_{a c}+\frac{1}{4} \operatorname{tr} T^{b}\left(D^{c}-T^{c}\right) T^{a}\right) \vec{I} \cdot \frac{z_{2}\left(\vec{p}_{2}-\vec{q}_{3}\right)-\left(1-z_{2}\right)\left(\vec{k}_{g}-\vec{q}_{2}\right)}{\left(z_{2}\left(\vec{p}_{2}-\vec{q}_{3}\right)-\left(1-z_{2}\right)\left(\vec{k}_{g}-\vec{q}_{2}\right)\right)^{2}} \\
& \times \Psi_{q q q}^{*}\left(x_{1}-x_{g}, \vec{k}_{1}+x_{1} \vec{q}-\vec{k}_{g}+x_{g} \vec{K} ; x_{2}+x_{g}, \vec{k}_{2}+x_{2} \vec{q}-\vec{q}_{23}+\vec{k}_{g}-x_{g} \vec{K} ; x_{3}, \vec{k}_{3}+x_{3} \vec{q}-\vec{q}_{1}\right) .
\end{aligned}
$$

The symmetry factor of 6 includes the contribution from diagram Fig.5 $\left(q_{1} g q_{3}\right)$ with the gluon emission and absorption vertices between quarks 1 and 2 interchanged,

$$
\begin{aligned}
\text { Fig.5 }\left(g q_{2} q_{3}\right)= & \left(\frac{1}{4}\left(T^{c}\right)_{a b}-\frac{N_{c}}{4}\left(D^{c}\right)_{a b}+\frac{1}{4} \operatorname{tr} T^{c}\left(D^{b}-T^{b}\right) T^{a}\right) \vec{I} \cdot \frac{z_{2}\left(\vec{p}_{2}-\vec{q}_{2}\right)-\left(1-z_{2}\right)\left(\vec{k}_{g}-\vec{q}_{3}\right)}{\left(z_{2}\left(\vec{p}_{2}-\vec{q}_{2}\right)-\left(1-z_{2}\right)\left(\vec{k}_{g}-\vec{q}_{3}\right)\right)^{2}} \\
& \times \Psi_{q q q}^{*}\left(x_{1}-x_{g}, \vec{k}_{1}+x_{1} \vec{q}-\vec{k}_{g}+x_{g} \vec{K} ; x_{2}+x_{g}, \vec{k}_{2}+x_{2} \vec{q}-\vec{q}_{23}+\vec{k}_{g}-x_{g} \vec{K} ; x_{3}, \vec{k}_{3}+x_{3} \vec{q}-\vec{q}_{1}\right) .
\end{aligned}
$$

The symmetry factor of 6 includes the contribution from diagram Fig.5 $\left(g q_{1} q_{3}\right)$ with the gluon emission and absorption vertices between quarks 1 and 2 interchanged,

Fig. $5\left(q_{3} q_{3} g\right)=0$,

Fig. $5\left(q_{3} g q_{3}\right)=0$,

Fig. $5\left(g q_{3} q_{3}\right)=0$. 


\section{Coupling only to quarks}

Here we consider the diagrams where all three gluon probes couple exclusively to quarks in the proton. We begin with the matrix element of $\rho^{a}\left(\vec{q}_{1}\right) \rho^{b}\left(\vec{q}_{2}\right) \rho^{c}\left(\vec{q}_{3}\right)$ in the $|q q q g\rangle$ Fock state. A few examples are shown in Fig. 6,

$$
\begin{aligned}
\text { Fig.6 }\left(q_{1} q_{1} q_{1}\right)= & -C_{F} \operatorname{tr} t^{a} t^{b} t^{c} \vec{I} \cdot \frac{z_{2} \vec{p}_{2}-\left(1-z_{2}\right) \vec{k}_{g}}{\left(z_{2} \vec{p}_{2}-\left(1-z_{2}\right) \vec{k}_{g}\right)^{2}} \\
& \times \Psi_{q q q}^{*}\left(x_{1}-x_{g}, \vec{k}_{1}-\left(1-x_{1}\right) \vec{q}-\vec{k}_{g}+x_{g} \vec{K} ; x_{2}+x_{g}, \vec{k}_{2}+x_{2} \vec{q}+\vec{k}_{g}-x_{g} \vec{K} ; x_{3}, \vec{k}_{3}+x_{3} \vec{q}\right) .
\end{aligned}
$$

The symmetry factor of 6 includes the contribution from diagram Fig. $6\left(q_{2} q_{2} q_{2}\right)$ with the gluon emission and absorption vertices between quarks 1 and 2 interchanged,

$$
\begin{aligned}
\text { Fig.6 }\left(q_{2} q_{1} q_{1}\right)= & -\left(C_{F}-\frac{1}{2}\right) \operatorname{tr} t^{a} t^{b} t^{c} \vec{I} \cdot \frac{z_{2}\left(\vec{p}_{2}-\vec{q}_{3}\right)-\left(1-z_{2}\right) \vec{k}_{g}}{\left(z_{2}\left(\vec{p}_{2}-\vec{q}_{3}\right)-\left(1-z_{2}\right) \vec{k}_{g}\right)^{2}} \\
& \times \Psi_{q q q}^{*}\left(x_{1}-x_{g}, \vec{k}_{1}+x_{1} \vec{q}-\vec{q}_{12}-\vec{k}_{g}+x_{g} \vec{K}_{2} x_{2}+x_{g}, \vec{k}_{2}+x_{2} \vec{q}-\vec{q}_{3}+\vec{k}_{g}-x_{g} \vec{K} ; x_{3}, \vec{k}_{3}+x_{3} \vec{q}\right) .
\end{aligned}
$$

The symmetry factor of 6 includes the contribution from diagram Fig.6 $\left(q_{1} q_{2} q_{2}\right)$ with the gluon emission and absorption vertices between quarks 1 and 2 interchanged,

$$
\begin{aligned}
\text { Fig.6 }\left(q_{3} q_{1} q_{1}\right)= & \left(C_{F}-\frac{1}{2}-\frac{1}{2 N_{c}}\right) \operatorname{tr} t^{a} t^{b} t^{c} \vec{I} \cdot \frac{z_{2} \vec{p}_{2}-\left(1-z_{2}\right) \vec{k}_{g}}{\left(z_{2} \vec{p}_{2}-\left(1-z_{2}\right) \vec{k}_{g}\right)^{2}} \\
& \times \Psi_{q q q}^{*}\left(x_{1}-x_{g}, \vec{k}_{1}+x_{1} \vec{q}-\vec{q}_{12}-\vec{k}_{g}+x_{g} \vec{K}_{2} ; x_{2}+x_{g}, \vec{k}_{2}+x_{2} \vec{q}+\vec{k}_{g}-x_{g} \vec{K} ; x_{3}, \vec{k}_{3}+x_{3} \vec{q}-\vec{q}_{3}\right) .
\end{aligned}
$$

The symmetry factor of 6 includes the contribution from diagram Fig. $6\left(q_{3} q_{2} q_{2}\right)$ with the gluon emission and absorption vertices between quarks 1 and 2 interchanged,

$$
\begin{aligned}
\text { Fig.6 }\left(q_{1} q_{2} q_{1}\right)= & -\left(C_{F}-\frac{1}{2}\right) \operatorname{tr} t^{a} t^{c} t^{b} \vec{I} \cdot \frac{z_{2}\left(\vec{p}_{2}-\vec{q}_{2}\right)-\left(1-z_{2}\right) \vec{k}_{g}}{\left(z_{2}\left(\vec{p}_{2}-\vec{q}_{2}\right)-\left(1-z_{2}\right) \vec{k}_{g}\right)^{2}} \\
& \times \Psi_{q q q}^{*}\left(x_{1}-x_{g}, \vec{k}_{1}+x_{1} \vec{q}-\vec{q}_{13}-\vec{k}_{g}+x_{g} \vec{K} ; x_{2}+x_{g}, \vec{k}_{2}+x_{2} \vec{q}-\vec{q}_{2}+\vec{k}_{g}-x_{g} \vec{K} ; x_{3}, \vec{k}_{3}+x_{3} \vec{q}\right) .
\end{aligned}
$$

The symmetry factor of 6 includes the contribution from diagram Fig.6 $\left(q_{2} q_{1} q_{2}\right)$ with the gluon emission and absorption vertices between quarks 1 and 2 interchanged,

$$
\begin{aligned}
\text { Fig.6 }\left(q_{2} q_{2} q_{1}\right)= & -\left(C_{F}-\frac{1}{2}\right) \operatorname{tr} t^{a} t^{b} t^{c} \vec{I} \cdot \frac{z_{2}\left(\vec{p}_{2}-\vec{q}_{23}\right)-\left(1-z_{2}\right) \vec{k}_{g}}{\left(z_{2}\left(\vec{p}_{2}-\vec{q}_{23}\right)-\left(1-z_{2}\right) \vec{k}_{g}\right)^{2}} \\
& \times \Psi_{q q q}^{*}\left(x_{1}-x_{g}, \vec{k}_{1}+x_{1} \vec{q}-\vec{q}_{1}-\vec{k}_{g}+x_{g} \vec{K} ; x_{2}+x_{g}, \vec{k}_{2}+x_{2} \vec{q}-\vec{q}_{23}+\vec{k}_{g}-x_{g} \vec{K} ; x_{3}, \vec{k}_{3}+x_{3} \vec{q}\right) .
\end{aligned}
$$
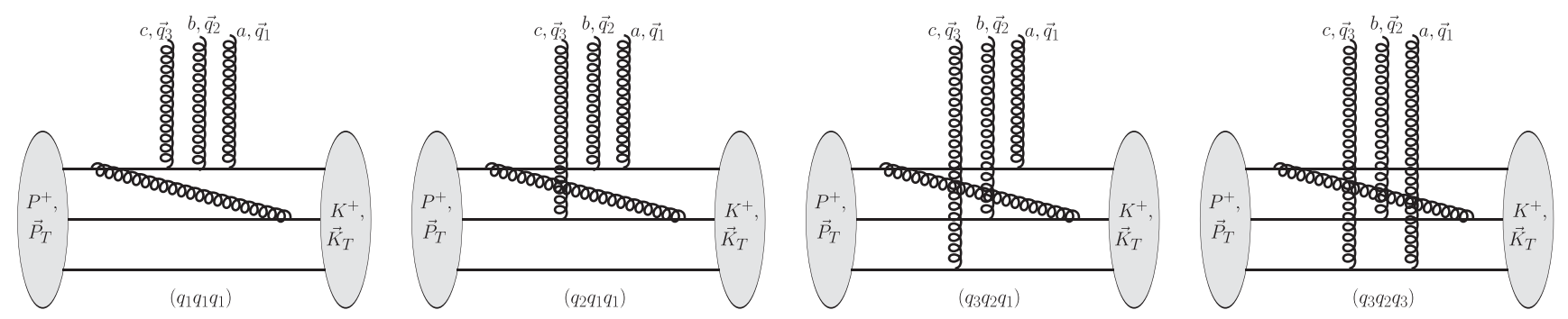

FIG. 6. A sample of UV finite diagrams for $\left\langle\rho^{a}\left(\vec{q}_{1}\right) \rho^{b}\left(\vec{q}_{2}\right) \rho^{c}\left(\vec{q}_{3}\right)\right\rangle$ evaluated in the $|q q q g\rangle$ Fock state, where all three probes attach to quarks in the proton. The cut is located at the insertion of the three color charge operators. 
The symmetry factor of 6 includes the contribution from diagram Fig.6 $\left(q_{1} q_{1} q_{2}\right)$ with the gluon emission and absorption vertices between quarks 1 and 2 interchanged,

$$
\begin{aligned}
\text { Fig.6 }\left(q_{3} q_{2} q_{1}\right)= & {\left[\left(C_{F}-1\right) \operatorname{tr} t^{a} t^{b} t^{c}+\left(C_{F}-\frac{N_{c}}{2}\right) \operatorname{tr} t^{a} t^{c} t^{b}\right] \vec{I} \cdot \frac{z_{2}\left(\vec{p}_{2}-\vec{q}_{2}\right)-\left(1-z_{2}\right) \vec{k}_{g}}{\left(z_{2}\left(\vec{p}_{2}-\vec{q}_{2}\right)-\left(1-z_{2}\right) \vec{k}_{g}\right)^{2}} } \\
& \times \Psi_{q q q}^{*}\left(x_{1}-x_{g}, \vec{k}_{1}+x_{1} \vec{q}-\vec{q}_{1}-\vec{k}_{g}+x_{g} \vec{K} ; x_{2}+x_{g}, \vec{k}_{2}+x_{2} \vec{q}-\vec{q}_{2}+\vec{k}_{g}-x_{g} \vec{K} ; x_{3}, \vec{k}_{3}+x_{3} \vec{q}-\vec{q}_{3}\right) .
\end{aligned}
$$

The symmetry factor of 6 includes the contribution from diagram Fig.6 $\left(q_{3} q_{1} q_{2}\right)$ with the gluon emission and absorption vertices between quarks 1 and 2 interchanged,

$$
\begin{aligned}
\text { Fig.6 }\left(q_{1} q_{3} q_{1}\right)= & \left(2 C_{F}-\frac{1}{2}-\frac{N_{c}}{2}\right) \operatorname{tr} t^{a} t^{c} t^{b} \vec{I} \cdot \frac{z_{2} \vec{p}_{2}-\left(1-z_{2}\right) \vec{k}_{g}}{\left(z_{2} \vec{p}_{2}-\left(1-z_{2}\right) \vec{k}_{g}\right)^{2}} \\
& \times \Psi_{q q q}^{*}\left(x_{1}-x_{g}, \vec{k}_{1}+x_{1} \vec{q}-\vec{q}_{13}-\vec{k}_{g}+x_{g} \vec{K} ; x_{2}+x_{g}, \vec{k}_{2}+x_{2} \vec{q}+\vec{k}_{g}-x_{g} \vec{K} ; x_{3}, \vec{k}_{3}+x_{3} \vec{q}-\vec{q}_{2}\right) .
\end{aligned}
$$

The symmetry factor of 6 includes the contribution from diagram Fig.6 $6\left(q_{2} q_{3} q_{2}\right)$ with the gluon emission and absorption vertices between quarks 1 and 2 interchanged,

$$
\begin{aligned}
\text { Fig.6 }\left(q_{2} q_{3} q_{1}\right)= & {\left[\left(C_{F}-1\right) \operatorname{tr} t^{a} t^{c} t^{b}+\left(C_{F}-\frac{N_{c}}{2}\right) \operatorname{tr} t^{a} t^{b} t^{c}\right] \vec{I} \cdot \frac{z_{2}\left(\vec{p}_{2}-\vec{q}_{3}\right)-\left(1-z_{2}\right) \vec{k}_{g}}{\left(z_{2}\left(\vec{p}_{2}-\vec{q}_{3}\right)-\left(1-z_{2}\right) \vec{k}_{g}\right)^{2}} } \\
& \times \Psi_{q q q}^{*}\left(x_{1}-x_{g}, \vec{k}_{1}+x_{1} \vec{q}-\vec{q}_{1}-\vec{k}_{g}+x_{g} \vec{K} ; x_{2}+x_{g}, \vec{k}_{2}+x_{2} \vec{q}-\vec{q}_{3}+\vec{k}_{g}-x_{g} \vec{K} ; x_{3}, \vec{k}_{3}+x_{3} \vec{q}-\vec{q}_{2}\right) .
\end{aligned}
$$

The symmetry factor of 6 includes the contribution from diagram Fig.6 $6\left(q_{1} q_{3} q_{2}\right)$ with the gluon emission and absorption vertices between quarks 1 and 2 interchanged,

$$
\begin{aligned}
\text { Fig.6 }\left(q_{3} q_{3} q_{1}\right)= & \left(2 C_{F}-\frac{1}{2}-\frac{N_{c}}{2}\right) \operatorname{tr} t^{a} t^{b} t^{c} \vec{I} \cdot \frac{z_{2} \vec{p}_{2}-\left(1-z_{2}\right) \vec{k}_{g}}{\left(z_{2} \vec{p}_{2}-\left(1-z_{2}\right) \vec{k}_{g}\right)^{2}} \\
& \times \Psi_{q q q}^{*}\left(x_{1}-x_{g}, \vec{k}_{1}+x_{1} \vec{q}-\vec{q}_{1}-\vec{k}_{g}+x_{g} \vec{K} ; x_{2}+x_{g}, \vec{k}_{2}+x_{2} \vec{q}+\vec{k}_{g}-x_{g} \vec{K} ; x_{3}, \vec{k}_{3}+x_{3} \vec{q}-\vec{q}_{23}\right) .
\end{aligned}
$$

The symmetry factor of 6 includes the contribution from diagram Fig.6 $\left(q_{3} q_{3} q_{2}\right)$ with the gluon emission and absorption vertices between quarks 1 and 2 interchanged,

$$
\begin{aligned}
\text { Fig.6 }\left(q_{1} q_{1} q_{2}\right)= & -\left(C_{F}-\frac{1}{2}\right) \operatorname{tr} t^{a} t^{b} t^{c} \vec{I} \cdot \frac{z_{2}\left(\vec{p}_{2}-\vec{q}_{1}\right)-\left(1-z_{2}\right) \vec{k}_{g}}{\left(z_{2}\left(\vec{p}_{2}-\vec{q}_{1}\right)-\left(1-z_{2}\right) \vec{k}_{g}\right)^{2}} \\
& \times \Psi_{q q q}^{*}\left(x_{1}-x_{g}, \vec{k}_{1}+x_{1} \vec{q}-\vec{q}_{23}-\vec{k}_{g}+x_{g} \vec{K} ; x_{2}+x_{g}, \vec{k}_{2}+x_{2} \vec{q}-\vec{q}_{1}+\vec{k}_{g}-x_{g} \vec{K} ; x_{3}, \vec{k}_{3}+x_{3} \vec{q}\right) .
\end{aligned}
$$

The symmetry factor of 6 includes the contribution from diagram Fig.6 $6\left(q_{2} q_{2} q_{1}\right)$ with the gluon emission and absorption vertices between quarks 1 and 2 interchanged,

$$
\begin{aligned}
\text { Fig.6 }\left(q_{2} q_{1} q_{2}\right)= & -\left(C_{F}-\frac{1}{2}\right) \operatorname{tr} t^{a} t^{c} t^{b} \vec{I} \cdot \frac{z_{2}\left(\vec{p}_{2}-\vec{q}_{13}\right)-\left(1-z_{2}\right) \vec{k}_{g}}{\left(z_{2}\left(\vec{p}_{2}-\vec{q}_{13}\right)-\left(1-z_{2}\right) \vec{k}_{g}\right)^{2}} \\
& \times \Psi_{q q q}^{*}\left(x_{1}-x_{g}, \vec{k}_{1}+x_{1} \vec{q}-\vec{q}_{2}-\vec{k}_{g}+x_{g} \vec{K} ; x_{2}+x_{g}, \vec{k}_{2}+x_{2} \vec{q}-\vec{q}_{13}+\vec{k}_{g}-x_{g} \vec{K} ; x_{3}, \vec{k}_{3}+x_{3} \vec{q}\right) .
\end{aligned}
$$

The symmetry factor of 6 includes the contribution from diagram Fig.6 $6\left(q_{1} q_{2} q_{1}\right)$ with the gluon emission and absorption vertices between quarks 1 and 2 interchanged, 


$$
\begin{aligned}
& \text { Fig.6 } 6\left(q_{3} q_{1} q_{2}\right)=\left[\left(C_{F}-\frac{N_{c}}{2}\right) \operatorname{tr} t^{a} t^{b} t^{c}+\left(C_{F}-1\right) \operatorname{tr} t^{a} t^{c} t^{b}\right] \vec{I} \cdot \frac{z_{2}\left(\vec{p}_{2}-\vec{q}_{1}\right)-\left(1-z_{2}\right) \vec{k}_{g}}{\left(z_{2}\left(\vec{p}_{2}-\vec{q}_{1}\right)-\left(1-z_{2}\right) \vec{k}_{g}\right)^{2}} \\
& \times \Psi_{q q q}^{*}\left(x_{1}-x_{g}, \vec{k}_{1}+x_{1} \vec{q}-\vec{q}_{2}-\vec{k}_{g}+x_{g} \vec{K} ; x_{2}+x_{g}, \vec{k}_{2}+x_{2} \vec{q}-\vec{q}_{1}+\vec{k}_{g}-x_{g} \vec{K} ; x_{3}, \vec{k}_{3}+x_{3} \vec{q}-\vec{q}_{3}\right) .
\end{aligned}
$$

The symmetry factor of 6 includes the contribution from diagram Fig. $6\left(q_{3} q_{2} q_{1}\right)$ with the gluon emission and absorption vertices between quarks 1 and 2 interchanged,

Fig. $6\left(q_{1} q_{2} q_{2}\right)=-\left(C_{F}-\frac{1}{2}\right) \operatorname{tr} t^{a} t^{b} t^{c} \vec{I} \cdot \frac{z_{2}\left(\vec{p}_{2}-\vec{q}_{12}\right)-\left(1-z_{2}\right) \vec{k}_{g}}{\left(z_{2}\left(\vec{p}_{2}-\vec{q}_{12}\right)-\left(1-z_{2}\right) \vec{k}_{g}\right)^{2}}$

$$
\times \Psi_{q q q}^{*}\left(x_{1}-x_{g}, \vec{k}_{1}+x_{1} \vec{q}-\vec{q}_{3}-\vec{k}_{g}+x_{g} \vec{K} ; x_{2}+x_{g}, \vec{k}_{2}+x_{2} \vec{q}-\vec{q}_{12}+\vec{k}_{g}-x_{g} \vec{K} ; x_{3}, \vec{k}_{3}+x_{3} \vec{q}\right) .
$$

The symmetry factor of 6 includes the contribution from diagram Fig. $6\left(q_{2} q_{1} q_{1}\right)$ with the gluon emission and absorption vertices between quarks 1 and 2 interchanged,

$$
\begin{aligned}
\text { Fig.6 }\left(q_{2} q_{2} q_{2}\right)= & -C_{F} \operatorname{tr} t^{a} t^{b} t^{c} \vec{I} \cdot \frac{z_{2}\left(\vec{p}_{2}-\vec{q}\right)-\left(1-z_{2}\right) \vec{k}_{g}}{\left(z_{2}\left(\vec{p}_{2}-\vec{q}\right)-\left(1-z_{2}\right) \vec{k}_{g}\right)^{2}} \\
& \times \Psi_{q q q}^{*}\left(x_{1}-x_{g}, \vec{k}_{1}+x_{1} \vec{q}-\vec{k}_{g}+x_{g} \vec{K} ; x_{2}+x_{g}, \vec{k}_{2}+x_{2} \vec{q}-\vec{q}+\vec{k}_{g}-x_{g} \vec{K} ; x_{3}, \vec{k}_{3}+x_{3} \vec{q}\right) .
\end{aligned}
$$

The symmetry factor of 6 includes the contribution from diagram Fig. $6\left(q_{1} q_{1} q_{1}\right)$ with the gluon emission and absorption vertices between quarks 1 and 2 interchanged,

Fig. $6\left(q_{3} q_{2} q_{2}\right)=\left(2 C_{F}-\frac{1}{2}-\frac{N_{c}}{2}\right) \operatorname{tr} t^{a} t^{b} t^{c} \vec{I} \cdot \frac{z_{2}\left(\vec{p}_{2}-\vec{q}_{12}\right)-\left(1-z_{2}\right) \vec{k}_{g}}{\left(z_{2}\left(\vec{p}_{2}-\vec{q}_{12}\right)-\left(1-z_{2}\right) \vec{k}_{g}\right)^{2}}$

$$
\times \Psi_{q q q}^{*}\left(x_{1}-x_{g}, \vec{k}_{1}+x_{1} \vec{q}-\vec{k}_{g}+x_{g} \vec{K} ; x_{2}+x_{g}, \vec{k}_{2}+x_{2} \vec{q}-\vec{q}_{12}+\vec{k}_{g}-x_{g} \vec{K} ; x_{3}, \vec{k}_{3}+x_{3} \vec{q}-\vec{q}_{3}\right) .
$$

The symmetry factor of 6 includes the contribution from diagram Fig. $6\left(q_{3} q_{1} q_{1}\right)$ with the gluon emission and absorption vertices between quarks 1 and 2 interchanged,

Fig.6 $6\left(q_{1} q_{3} q_{2}\right)=\left[\left(C_{F}-1\right) \operatorname{tr} t^{a} t^{b} t^{c}+\left(C_{F}-\frac{N_{c}}{2}\right) \operatorname{tr} t^{a} t^{c} t^{b}\right] \vec{I} \cdot \frac{z_{2}\left(\vec{p}_{2}-\vec{q}_{1}\right)-\left(1-z_{2}\right) \vec{k}_{g}}{\left(z_{2}\left(\vec{p}_{2}-\vec{q}_{1}\right)-\left(1-z_{2}\right) \vec{k}_{g}\right)^{2}}$

$$
\times \Psi_{q q q}^{*}\left(x_{1}-x_{g}, \vec{k}_{1}+x_{1} \vec{q}-\vec{q}_{3}-\vec{k}_{g}+x_{g} \vec{K} ; x_{2}+x_{g}, \vec{k}_{2}+x_{2} \vec{q}-\vec{q}_{1}+\vec{k}_{g}-x_{g} \vec{K} ; x_{3}, \vec{k}_{3}+x_{3} \vec{q}-\vec{q}_{2}\right) .
$$

The symmetry factor of 6 includes the contribution from diagram Fig. $6\left(q_{2} q_{3} q_{1}\right)$ with the gluon emission and absorption vertices between quarks 1 and 2 interchanged,

Fig. $6\left(q_{2} q_{3} q_{2}\right)=\left(2 C_{F}-\frac{1}{2}-\frac{N_{c}}{2}\right) \operatorname{tr} t^{a} t^{c} t^{b} \vec{I} \cdot \frac{z_{2}\left(\vec{p}_{2}-\vec{q}_{13}\right)-\left(1-z_{2}\right) \vec{k}_{g}}{\left(z_{2}\left(\vec{p}_{2}-\vec{q}_{13}\right)-\left(1-z_{2}\right) \vec{k}_{g}\right)^{2}}$

$$
\times \Psi_{q q q}^{*}\left(x_{1}-x_{g}, \vec{k}_{1}+x_{1} \vec{q}-\vec{k}_{g}+x_{g} \vec{K} ; x_{2}+x_{g}, \vec{k}_{2}+x_{2} \vec{q}-\vec{q}_{13}+\vec{k}_{g}-x_{g} \vec{K} ; x_{3}, \vec{k}_{3}+x_{3} \vec{q}-\vec{q}_{2}\right) .
$$

The symmetry factor of 6 includes the contribution from diagram Fig. $6\left(q_{1} q_{3} q_{1}\right)$ with the gluon emission and absorption vertices between quarks 1 and 2 interchanged,

Fig. $6\left(q_{3} q_{3} q_{2}\right)=\left(2 C_{F}-\frac{1}{2}-\frac{N_{c}}{2}\right) \operatorname{tr} t^{a} t^{b} t^{c} \vec{I} \cdot \frac{z_{2}\left(\vec{p}_{2}-\vec{q}_{1}\right)-\left(1-z_{2}\right) \vec{k}_{g}}{\left(z_{2}\left(\vec{p}_{2}-\vec{q}_{1}\right)-\left(1-z_{2}\right) \vec{k}_{g}\right)^{2}}$

$$
\times \Psi_{q q q}^{*}\left(x_{1}-x_{g}, \vec{k}_{1}+x_{1} \vec{q}-\vec{k}_{g}+x_{g} \vec{K} ; x_{2}+x_{g}, \vec{k}_{2}+x_{2} \vec{q}-\vec{q}_{1}+\vec{k}_{g}-x_{g} \vec{K} ; x_{3}, \vec{k}_{3}+x_{3} \vec{q}-\vec{q}_{23}\right) .
$$


The symmetry factor of 6 includes the contribution from diagram Fig.6 $\left(q_{3} q_{3} q_{1}\right)$ with the gluon emission and absorption vertices between quarks 1 and 2 interchanged,

Fig. $6\left(q_{1} q_{1} q_{3}\right)=\left(2 C_{F}-\frac{1}{2}-\frac{N_{c}}{2}\right) \operatorname{tr} t^{a} t^{b} t^{c} \vec{I} \cdot \frac{z_{2} \vec{p}_{2}-\left(1-z_{2}\right) \vec{k}_{g}}{\left(z_{2} \vec{p}_{2}-\left(1-z_{2}\right) \vec{k}_{g}\right)^{2}}$

$$
\times \Psi_{q q q}^{*}\left(x_{1}-x_{g}, \vec{k}_{1}+x_{1} \vec{q}-\vec{q}_{23}-\vec{k}_{g}+x_{g} \vec{K} ; x_{2}+x_{g}, \vec{k}_{2}+x_{2} \vec{q}+\vec{k}_{g}-x_{g} \vec{K} ; x_{3}, \vec{k}_{3}+x_{3} \vec{q}-\vec{q}_{1}\right) .
$$

The symmetry factor of 6 includes the contribution from diagram Fig. $6\left(q_{2} q_{2} q_{3}\right)$ with the gluon emission and absorption vertices between quarks 1 and 2 interchanged,

Fig. $6\left(q_{2} q_{1} q_{3}\right)=\left[\left(C_{F}-1\right) \operatorname{tr} t^{a} t^{b} t^{c}+\left(C_{F}-\frac{N_{c}}{2}\right) \operatorname{tr} t^{a} t^{c} t^{b}\right] \vec{I} \cdot \frac{z_{2}\left(\vec{p}_{2}-\vec{q}_{3}\right)-\left(1-z_{2}\right) \vec{k}_{g}}{\left(z_{2}\left(\vec{p}_{2}-\vec{q}_{3}\right)-\left(1-z_{2}\right) \vec{k}_{g}\right)^{2}}$

$$
\times \Psi_{q q q}^{*}\left(x_{1}-x_{g}, \vec{k}_{1}+x_{1} \vec{q}-\vec{q}_{2}-\vec{k}_{g}+x_{g} \vec{K} ; x_{2}+x_{g}, \vec{k}_{2}+x_{2} \vec{q}-\vec{q}_{3}+\vec{k}_{g}-x_{g} \vec{K} ; x_{3}, \vec{k}_{3}+x_{3} \vec{q}-\vec{q}_{1}\right) .
$$

The symmetry factor of 6 includes the contribution from diagram Fig. $6\left(q_{1} q_{2} q_{3}\right)$ with the gluon emission and absorption vertices between quarks 1 and 2 interchanged,

Fig. $6\left(q_{3} q_{1} q_{3}\right)=\left(2 C_{F}-\frac{1}{2}-\frac{N_{c}}{2}\right) \operatorname{tr} t^{a} t^{c} t^{b} \vec{I} \cdot \frac{z_{2} \vec{p}_{2}-\left(1-z_{2}\right) \vec{k}_{g}}{\left(z_{2} \vec{p}_{2}-\left(1-z_{2}\right) \vec{k}_{g}\right)^{2}}$

$$
\times \Psi_{q q q}^{*}\left(x_{1}-x_{g}, \vec{k}_{1}+x_{1} \vec{q}-\vec{q}_{2}-\vec{k}_{g}+x_{g} \vec{K} ; x_{2}+x_{g}, \vec{k}_{2}+x_{2} \vec{q}+\vec{k}_{g}-x_{g} \vec{K} ; x_{3}, \vec{k}_{3}+x_{3} \vec{q}-\vec{q}_{13}\right) .
$$

The symmetry factor of 6 includes the contribution from diagram Fig.6 $6\left(q_{3} q_{2} q_{3}\right)$ with the gluon emission and absorption vertices between quarks 1 and 2 interchanged,

Fig.6 $6\left(q_{1} q_{2} q_{3}\right)=\left[\left(C_{F}-1\right) \operatorname{tr} t^{a} t^{c} t^{b}+\left(C_{F}-\frac{N_{c}}{2}\right) \operatorname{tr} t^{a} t^{b} t^{c}\right] \vec{I} \cdot \frac{z_{2}\left(\vec{p}_{2}-\vec{q}_{2}\right)-\left(1-z_{2}\right) \vec{k}_{g}}{\left(z_{2}\left(\vec{p}_{2}-\vec{q}_{2}\right)-\left(1-z_{2}\right) \vec{k}_{g}\right)^{2}}$

$$
\times \Psi_{q q q}^{*}\left(x_{1}-x_{g}, \vec{k}_{1}+x_{1} \vec{q}-\vec{q}_{3}-\vec{k}_{g}+x_{g} \vec{K} ; x_{2}+x_{g}, \vec{k}_{2}+x_{2} \vec{q}-\vec{q}_{2}+\vec{k}_{g}-x_{g} \vec{K} ; x_{3}, \vec{k}_{3}+x_{3} \vec{q}-\vec{q}_{1}\right) .
$$

The symmetry factor of 6 includes the contribution from diagram Fig.6 $\left(q_{2} q_{1} q_{3}\right)$ with the gluon emission and absorption vertices between quarks 1 and 2 interchanged,

Fig. $6\left(q_{2} q_{2} q_{3}\right)=\left(2 C_{F}-\frac{1}{2}-\frac{N_{c}}{2}\right) \operatorname{tr} t^{a} t^{b} t^{c} \vec{I} \cdot \frac{z_{2}\left(\vec{p}_{2}-\vec{q}_{23}\right)-\left(1-z_{2}\right) \vec{k}_{g}}{\left(z_{2}\left(\vec{p}_{2}-\vec{q}_{23}\right)-\left(1-z_{2}\right) \vec{k}_{g}\right)^{2}}$

$$
\times \Psi_{q q q}^{*}\left(x_{1}-x_{g}, \vec{k}_{1}+x_{1} \vec{q}-\vec{k}_{g}+x_{g} \vec{K} ; x_{2}+x_{g}, \vec{k}_{2}+x_{2} \vec{q}-\vec{q}_{23}+\vec{k}_{g}-x_{g} \vec{K} ; x_{3}, \vec{k}_{3}+x_{3} \vec{q}-\vec{q}_{1}\right) .
$$

The symmetry factor of 6 includes the contribution from diagram Fig.6 $\left(q_{1} q_{1} q_{3}\right)$ with the gluon emission and absorption vertices between quarks 1 and 2 interchanged,

$\begin{aligned} \text { Fig.6 }\left(q_{3} q_{2} q_{3}\right)= & \left(2 C_{F}-\frac{1}{2}-\frac{N_{c}}{2}\right) \operatorname{tr} t^{a} t^{c} t^{b} \vec{I} \cdot \frac{z_{2}\left(\vec{p}_{2}-\vec{q}_{2}\right)-\left(1-z_{2}\right) \vec{k}_{g}}{\left(z_{2}\left(\vec{p}_{2}-\vec{q}_{2}\right)-\left(1-z_{2}\right) \vec{k}_{g}\right)^{2}} \\ & \times \Psi_{q q q}^{*}\left(x_{1}-x_{g}, \vec{k}_{1}+x_{1} \vec{q}-\vec{k}_{g}+x_{g} \vec{K} ; x_{2}+x_{g}, \vec{k}_{2}+x_{2} \vec{q}-\vec{q}_{2}+\vec{k}_{g}-x_{g} \vec{K} ; x_{3}, \vec{k}_{3}+x_{3} \vec{q}-\vec{q}_{13}\right) .\end{aligned}$

The symmetry factor of 6 includes the contribution from diagram fig.6 $\left(q_{3} q_{1} q_{3}\right)$ with the gluon emission and absorption vertices between quarks 1 and 2 interchanged. 


$$
\begin{aligned}
& \text { fig. } 6\left(q_{1} q_{3} q_{3}\right)=\left(2 C_{F}-\frac{1}{2}-\frac{N_{c}}{2}\right) \operatorname{tr} t^{a} t^{b} t^{c} \vec{I} \cdot \frac{z_{2} \vec{p}_{2}-\left(1-z_{2}\right) \vec{k}_{g}}{\left(z_{2} \vec{p}_{2}-\left(1-z_{2}\right) \vec{k}_{g}\right)^{2}} \\
& \times \Psi_{q q q}^{*}\left(x_{1}-x_{g}, \vec{k}_{1}+x_{1} \vec{q}-\vec{q}_{3}-\vec{k}_{g}+x_{g} \vec{K} ; x_{2}+x_{g}, \vec{k}_{2}+x_{2} \vec{q}+\vec{k}_{g}-x_{g} \vec{K} ; x_{3}, \vec{k}_{3}+x_{3} \vec{q}-\vec{q}_{12}\right) .
\end{aligned}
$$

The symmetry factor of 6 includes the contribution from diagram Fig.6 $\left(q_{2} q_{3} q_{3}\right)$ with the gluon emission and absorption vertices between quarks 1 and 2 interchanged,

$$
\begin{aligned}
\text { Fig.6 }\left(q_{2} q_{3} q_{3}\right)= & \left(2 C_{F}-\frac{1}{2}-\frac{N_{c}}{2}\right) \operatorname{tr} t^{a} t^{b} t^{c} \vec{I} \cdot \frac{z_{2}\left(\vec{p}_{2}-\vec{q}_{3}\right)-\left(1-z_{2}\right) \vec{k}_{g}}{\left(z_{2}\left(\vec{p}_{2}-\vec{q}_{3}\right)-\left(1-z_{2}\right) \vec{k}_{g}\right)^{2}} \\
& \times \Psi_{q q q}^{*}\left(x_{1}-x_{g}, \vec{k}_{1}+x_{1} \vec{q}-\vec{k}_{g}+x_{g} \vec{K} ; x_{2}+x_{g}, \vec{k}_{2}+x_{2} \vec{q}-\vec{q}_{3}+\vec{k}_{g}-x_{g} \vec{K} ; x_{3}, \vec{k}_{3}+x_{3} \vec{q}-\vec{q}_{12}\right) .
\end{aligned}
$$

The symmetry factor of 6 includes the contribution from diagram Fig. $6\left(q_{1} q_{3} q_{3}\right)$ with the gluon emission and absorption vertices between quarks 1 and 2 interchanged,

$$
\begin{aligned}
\text { Fig.6 }\left(q_{3} q_{3} q_{3}\right)= & C_{F}\left(2-N_{c}\right) \operatorname{tr} t^{a} t^{b} t^{c} \vec{I} \cdot \frac{z_{2} \vec{p}_{2}-\left(1-z_{2}\right) \vec{k}_{g}}{\left(z_{2} \vec{p}_{2}-\left(1-z_{2}\right) \vec{k}_{g}\right)^{2}} \\
& \times \Psi_{q q q}^{*}\left(x_{1}-x_{g}, \vec{k}_{1}+x_{1} \vec{q}-\vec{k}_{g}+x_{g} \vec{K} ; x_{2}+x_{g}, \vec{k}_{2}+x_{2} \vec{q}+\vec{k}_{g}-x_{g} \vec{K} ; x_{3}, \vec{k}_{3}+x_{3} \vec{q}-\vec{q}\right) .
\end{aligned}
$$

The symmetry factor of 6 includes the contribution from diagram Fig. $6\left(q_{3} q_{3} q_{3}\right)$ with the gluon emission and absorption vertices between quarks 1 and 2 interchanged.

The final set of diagrams corresponds to the virtual corrections where two quarks exchange a gluon on either side of the insertion of the $\rho^{a}\left(\vec{q}_{1}\right) \rho^{b}\left(\vec{q}_{2}\right) \rho^{c}\left(\vec{q}_{3}\right)$ operator. Figure 7 shows a small subset of these diagrams. To write them in compact form we introduce the integral operator

$$
\begin{aligned}
J= & \frac{g^{5}}{3 \cdot 16 \pi^{3}} \int\left[\mathrm{d} x_{i}\right] \int\left[\mathrm{d}^{2} k_{i}\right] \Psi_{q q q}\left(x_{1}, \vec{k}_{1} ; x_{2}, \vec{k}_{2} ; x_{3}, \vec{k}_{3}\right) \\
& \times \int_{x}^{\min \left(x_{1}, 1-x_{2}\right)} \frac{\mathrm{d} x_{g}}{x_{g}}\left(1-\frac{z_{1}+z_{2}}{2}+\frac{z_{1} z_{2}}{6}\right) \sqrt{\frac{x_{1}}{x_{1}-x_{g}}} \sqrt{\frac{x_{2}}{x_{2}+x_{g}}} \int \mathrm{d}^{2} k_{g} .
\end{aligned}
$$

We shall also include right away the contribution from the diagram where the gluon exchange occurs on the other side of the insertion from quark 2 to quark 1 . With this,

$$
\begin{aligned}
& \text { Fig.7 }\left(q_{1} q_{1} q_{1}\right)=C_{F} \operatorname{tr} t^{a} t^{b} t^{c} J \frac{z_{2} \vec{p}_{2}-\left(1-z_{2}\right) \vec{k}_{g}}{\left(z_{2} \vec{p}_{2}-\left(1-z_{2}\right) \vec{k}_{g}\right)^{2}} \cdot\left(\frac{z_{1} \vec{p}_{1}-\vec{k}_{g}}{\left(z_{1} \vec{p}_{1}-\vec{k}_{g}\right)^{2}}+\frac{z_{1}\left(\vec{p}_{1}-\vec{q}\right)-\vec{k}_{g}}{\left(z_{1}\left(\vec{p}_{1}-\vec{q}\right)-\vec{k}_{g}\right)^{2}}\right) \\
& \times \Psi_{q q q}^{*}\left(x_{1}-x_{g}, \vec{k}_{1}+x_{1} \vec{q}-\vec{q}-\vec{k}_{g}+x_{g} \vec{K} ; x_{2}+x_{g}, \vec{k}_{2}+x_{2} \vec{q}+\vec{k}_{g}-x_{g} \vec{K} ; x_{3}, \vec{k}_{3}+x_{3} \vec{q}\right) .
\end{aligned}
$$

The symmetry factor of 6 includes the contribution from diagram Fig. $7\left(q_{2} q_{2} q_{2}\right)$ with the gluon emission and absorption vertices between quarks 1 and 2 interchanged.
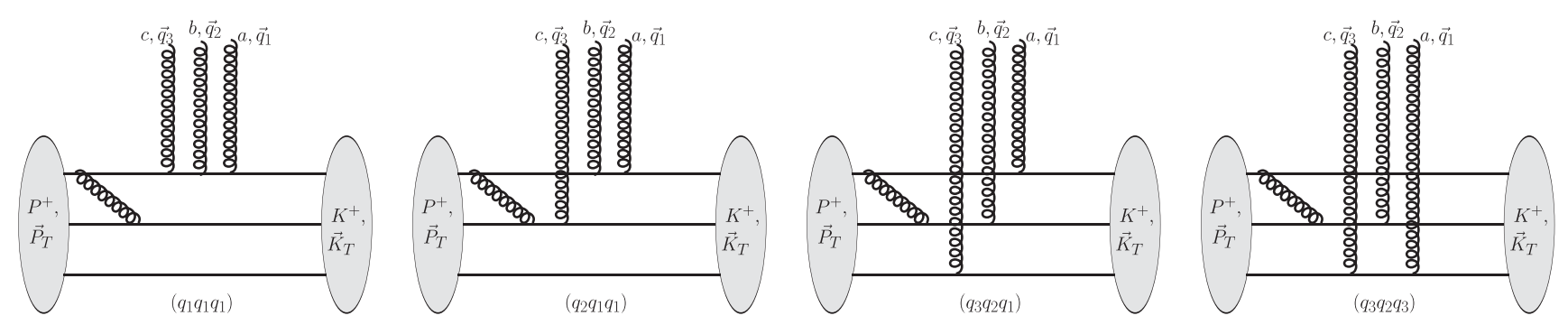

FIG. 7. A sample of UV finite diagrams for $\left\langle\rho^{a}\left(\vec{q}_{1}\right) \rho^{b}\left(\vec{q}_{2}\right) \rho^{c}\left(\vec{q}_{3}\right)\right\rangle$ evaluated in the $|q q q\rangle$ Fock state including $\mathcal{O}\left(g^{2}\right)$ virtual correction. The cut is located at the insertion of the three color charge operators. 
Fig. $7\left(q_{2} q_{1} q_{1}\right)=\left(C_{F}-\frac{N_{c}+1}{2}\right) \operatorname{tr} t^{a} t^{b} t^{c} J\left(\frac{z_{2} \vec{p}_{2}-\left(1-z_{2}\right) \vec{k}_{g}}{\left(z_{2} \vec{p}_{2}-\left(1-z_{2}\right) \vec{k}_{g}\right)^{2}} \cdot \frac{z_{1} \vec{p}_{1}-\vec{k}_{g}}{\left(z_{1} \vec{p}_{1}-\vec{k}_{g}\right)^{2}}\right.$

$$
\begin{aligned}
& \left.+\frac{z_{2}\left(\vec{p}_{2}-\vec{q}_{3}\right)-\left(1-z_{2}\right) \vec{k}_{g}}{\left(z_{2}\left(\vec{p}_{2}-\vec{q}_{3}\right)-\left(1-z_{2}\right) \vec{k}_{g}\right)^{2}} \cdot \frac{z_{1}\left(\vec{p}_{1}-\vec{q}_{12}\right)-\vec{k}_{g}}{\left(z_{1}\left(\vec{p}_{1}-\vec{q}_{12}\right)-\vec{k}_{g}\right)^{2}}\right) \\
& \times \Psi_{q q q}^{*}\left(x_{1}-x_{g}, \vec{k}_{1}+x_{1} \vec{q}-\vec{q}_{12}-\vec{k}_{g}+x_{g} \vec{K} ; x_{2}+x_{g}, \vec{k}_{2}+x_{2} \vec{q}-\vec{q}_{3}+\vec{k}_{g}-x_{g} \vec{K} ; x_{3}, \vec{k}_{3}+x_{3} \vec{q}\right) .
\end{aligned}
$$

The symmetry factor of 6 includes the contribution from diagram Fig. $7\left(q_{1} q_{2} q_{2}\right)$ with the gluon emission and absorption vertices between quarks 1 and 2 interchanged.

Fig.7 $\left(q_{3} q_{1} q_{1}\right)=-\left(2 C_{F}-\frac{N_{c}+1}{2}\right) \operatorname{tr} t^{a} t^{b} t^{c} J \frac{z_{2} \vec{p}_{2}-\left(1-z_{2}\right) \vec{k}_{g}}{\left(z_{2} \vec{p}_{2}-\left(1-z_{2}\right) \vec{k}_{g}\right)^{2}} \cdot\left(\frac{z_{1} \vec{p}_{1}-\vec{k}_{g}}{\left(z_{1} \vec{p}_{1}-\vec{k}_{g}\right)^{2}}+\frac{z_{1}\left(\vec{p}_{1}-\vec{q}_{12}\right)-\vec{k}_{g}}{\left(z_{1}\left(\vec{p}_{1}-\vec{q}_{12}\right)-\vec{k}_{g}\right)^{2}}\right)$

$$
\times \Psi_{q q q}^{*}\left(x_{1}-x_{g}, \vec{k}_{1}+x_{1} \vec{q}-\vec{q}_{12}-\vec{k}_{g}+x_{g} \vec{K} ; x_{2}+x_{g}, \vec{k}_{2}+x_{2} \vec{q}+\vec{k}_{g}-x_{g} \vec{K} ; x_{3}, \vec{k}_{3}+x_{3} \vec{q}-\vec{q}_{3}\right) .
$$

The symmetry factor of 6 includes the contribution from diagram Fig. $7\left(q_{3} q_{2} q_{2}\right)$ with the gluon emission and absorption vertices between quarks 1 and 2 interchanged.

Fig.7 $\left(q_{1} q_{2} q_{1}\right)=\left(C_{F}-\frac{N_{c}+1}{2}\right) \operatorname{tr} t^{a} t^{c} t^{b} J\left(\frac{z_{2} \vec{p}_{2}-\left(1-z_{2}\right) \vec{k}_{g}}{\left(z_{2} \vec{p}_{2}-\left(1-z_{2}\right) \vec{k}_{g}\right)^{2}} \cdot \frac{z_{1} \vec{p}_{1}-\vec{k}_{g}}{\left(z_{1} \vec{p}_{1}-\vec{k}_{g}\right)^{2}}\right.$

$$
\begin{aligned}
& \left.+\frac{z_{2}\left(\vec{p}_{2}-\vec{q}_{2}\right)-\left(1-z_{2}\right) \vec{k}_{g}}{\left(z_{2}\left(\vec{p}_{2}-\vec{q}_{2}\right)-\left(1-z_{2}\right) \vec{k}_{g}\right)^{2}} \cdot \frac{z_{1}\left(\vec{p}_{1}-\vec{q}_{13}\right)-\vec{k}_{g}}{\left(z_{1}\left(\vec{p}_{1}-\vec{q}_{13}\right)-\vec{k}_{g}\right)^{2}}\right) \\
& \times \Psi_{q q q}^{*}\left(x_{1}-x_{g}, \vec{k}_{1}+x_{1} \vec{q}-\vec{q}_{13}-\vec{k}_{g}+x_{g} \vec{K}^{\prime} x_{2}+x_{g}, \vec{k}_{2}+x_{2} \vec{q}-\vec{q}_{2}+\vec{k}_{g}-x_{g} \vec{K} ; x_{3}, \vec{k}_{3}+x_{3} \vec{q}\right) .
\end{aligned}
$$

The symmetry factor of 6 includes the contribution from diagram Fig. $7\left(q_{2} q_{1} q_{2}\right)$ with the gluon emission and absorption vertices between quarks 1 and 2 interchanged.

Fig. $7\left(q_{2} q_{2} q_{1}\right)=\left(C_{F}-\frac{N_{c}+1}{2}\right) \operatorname{tr} t^{a} t^{b} t^{c} J\left(\frac{z_{2} \vec{p}_{2}-\left(1-z_{2}\right) \vec{k}_{g}}{\left(z_{2} \vec{p}_{2}-\left(1-z_{2}\right) \vec{k}_{g}\right)^{2}} \cdot \frac{z_{1} \vec{p}_{1}-\vec{k}_{g}}{\left(z_{1} \vec{p}_{1}-\vec{k}_{g}\right)^{2}}\right.$

$$
\begin{aligned}
& \left.+\frac{z_{2}\left(\vec{p}_{2}-\vec{q}_{23}\right)-\left(1-z_{2}\right) \vec{k}_{g}}{\left(z_{2}\left(\vec{p}_{2}-\vec{q}_{23}\right)-\left(1-z_{2}\right) \vec{k}_{g}\right)^{2}} \cdot \frac{z_{1}\left(\vec{p}_{1}-\vec{q}_{1}\right)-\vec{k}_{g}}{\left(z_{1}\left(\vec{p}_{1}-\vec{q}_{1}\right)-\vec{k}_{g}\right)^{2}}\right) \\
& \times \Psi_{q q q}^{*}\left(x_{1}-x_{g}, \vec{k}_{1}+x_{1} \vec{q}-\vec{q}_{1}-\vec{k}_{g}+x_{g} \vec{K} ; x_{2}+x_{g}, \vec{k}_{2}+x_{2} \vec{q}-\vec{q}_{23}+\vec{k}_{g}-x_{g} \vec{K} ; x_{3}, \vec{k}_{3}+x_{3} \vec{q}\right) .
\end{aligned}
$$

The symmetry factor of 6 includes the contribution from diagram Fig. $7\left(q_{1} q_{1} q_{2}\right)$ with the gluon emission and absorption vertices between quarks 1 and 2 interchanged.

Fig. $7\left(q_{3} q_{2} q_{1}\right)=-\left(C_{F}-\frac{N_{c}+1}{2}\right)\left(\operatorname{tr} t^{a} t^{b} t^{c}+\operatorname{tr} t^{a} t^{c} t^{b}\right) J\left(\frac{z_{2} \vec{p}_{2}-\left(1-z_{2}\right) \vec{k}_{g}}{\left(z_{2} \vec{p}_{2}-\left(1-z_{2}\right) \vec{k}_{g}\right)^{2}} \cdot \frac{z_{1} \vec{p}_{1}-\vec{k}_{g}}{\left(z_{1} \vec{p}_{1}-\vec{k}_{g}\right)^{2}}\right.$

$$
\begin{aligned}
& \left.+\frac{z_{2}\left(\vec{p}_{2}-\vec{q}_{2}\right)-\left(1-z_{2}\right) \vec{k}_{g}}{\left(z_{2}\left(\vec{p}_{2}-\vec{q}_{2}\right)-\left(1-z_{2}\right) \vec{k}_{g}\right)^{2}} \cdot \frac{z_{1}\left(\vec{p}_{1}-\vec{q}_{1}\right)-\vec{k}_{g}}{\left(z_{1}\left(\vec{p}_{1}-\vec{q}_{1}\right)-\vec{k}_{g}\right)^{2}}\right) \\
& \times \Psi_{q q q}^{*}\left(x_{1}-x_{g}, \vec{k}_{1}+x_{1} \vec{q}-\vec{q}_{1}-\vec{k}_{g}+x_{g} \vec{K} ; x_{2}+x_{g}, \vec{k}_{2}+x_{2} \vec{q}-\vec{q}_{2}+\vec{k}_{g}-x_{g} \vec{K} ; x_{3}, \vec{k}_{3}+x_{3} \vec{q}-\vec{q}_{3}\right) .
\end{aligned}
$$

The symmetry factor of 6 includes the contribution from diagram Fig. $7\left(q_{3} q_{1} q_{2}\right)$ with the gluon emission and absorption vertices between quarks 1 and 2 interchanged. 
Fig.7 $\left(q_{1} q_{3} q_{1}\right)=-\left(2 C_{F}-\frac{N_{c}+1}{2}\right) \operatorname{tr} t^{a} t^{c} t^{b} J \frac{z_{2} \vec{p}_{2}-\left(1-z_{2}\right) \vec{k}_{g}}{\left(z_{2} \vec{p}_{2}-\left(1-z_{2}\right) \vec{k}_{g}\right)^{2}} \cdot\left(\frac{z_{1} \vec{p}_{1}-\vec{k}_{g}}{\left(z_{1} \vec{p}_{1}-\vec{k}_{g}\right)^{2}}+\frac{z_{1}\left(\vec{p}_{1}-\vec{q}_{13}\right)-\vec{k}_{g}}{\left(z_{1}\left(\vec{p}_{1}-\vec{q}_{13}\right)-\vec{k}_{g}\right)^{2}}\right)$

$$
\times \Psi_{q q q}^{*}\left(x_{1}-x_{g}, \vec{k}_{1}+x_{1} \vec{q}-\vec{q}_{13}-\vec{k}_{g}+x_{g} \vec{K} ; x_{2}+x_{g}, \vec{k}_{2}+x_{2} \vec{q}+\vec{k}_{g}-x_{g} \vec{K} ; x_{3}, \vec{k}_{3}+x_{3} \vec{q}-\vec{q}_{2}\right) .
$$

The symmetry factor of 6 includes the contribution from diagram Fig. $7\left(q_{2} q_{3} q_{2}\right)$ with the gluon emission and absorption vertices between quarks 1 and 2 interchanged.

Fig. $7\left(q_{2} q_{3} q_{1}\right)=-\left(C_{F}-\frac{N_{c}+1}{2}\right)\left(\operatorname{tr} t^{a} t^{b} t^{c}+\operatorname{tr} t^{a} t^{c} t^{b}\right) J\left(\frac{z_{2} \vec{p}_{2}-\left(1-z_{2}\right) \vec{k}_{g}}{\left(z_{2} \vec{p}_{2}-\left(1-z_{2}\right) \vec{k}_{g}\right)^{2}} \cdot \frac{z_{1} \vec{p}_{1}-\vec{k}_{g}}{\left(z_{1} \vec{p}_{1}-\vec{k}_{g}\right)^{2}}\right.$

$$
\begin{aligned}
& \left.+\frac{z_{2}\left(\vec{p}_{2}-\vec{q}_{3}\right)-\left(1-z_{2}\right) \vec{k}_{g}}{\left(z_{2}\left(\vec{p}_{2}-\vec{q}_{3}\right)-\left(1-z_{2}\right) \vec{k}_{g}\right)^{2}} \cdot \frac{z_{1}\left(\vec{p}_{1}-\vec{q}_{1}\right)-\vec{k}_{g}}{\left(z_{1}\left(\vec{p}_{1}-\vec{q}_{1}\right)-\vec{k}_{g}\right)^{2}}\right) \\
& \times \Psi_{q q q}^{*}\left(x_{1}-x_{g}, \vec{k}_{1}+x_{1} \vec{q}-\vec{q}_{1}-\vec{k}_{g}+x_{g} \vec{K} ; x_{2}+x_{g}, \vec{k}_{2}+x_{2} \vec{q}-\vec{q}_{3}+\vec{k}_{g}-x_{g} \vec{K} ; x_{3}, \vec{k}_{3}+x_{3} \vec{q}-\vec{q}_{2}\right) .
\end{aligned}
$$

The symmetry factor of 6 includes the contribution from diagram Fig. $7\left(q_{1} q_{3} q_{2}\right)$ with the gluon emission and absorption vertices between quarks 1 and 2 interchanged.

Fig.7 $\left(q_{3} q_{3} q_{1}\right)=-\left(2 C_{F}-\frac{N_{c}+1}{2}\right) \operatorname{tr} t^{a} t^{b} t^{c} J \frac{z_{2} \vec{p}_{2}-\left(1-z_{2}\right) \vec{k}_{g}}{\left(z_{2} \vec{p}_{2}-\left(1-z_{2}\right) \vec{k}_{g}\right)^{2}} \cdot\left(\frac{z_{1} \vec{p}_{1}-\vec{k}_{g}}{\left(z_{1} \vec{p}_{1}-\vec{k}_{g}\right)^{2}}+\frac{z_{1}\left(\vec{p}_{1}-\vec{q}_{1}\right)-\vec{k}_{g}}{\left(z_{1}\left(\vec{p}_{1}-\vec{q}_{1}\right)-\vec{k}_{g}\right)^{2}}\right)$

$$
\times \Psi_{q q q}^{*}\left(x_{1}-x_{g}, \vec{k}_{1}+x_{1} \vec{q}-\vec{q}_{1}-\vec{k}_{g}+x_{g} \vec{K} ; x_{2}+x_{g}, \vec{k}_{2}+x_{2} \vec{q}+\vec{k}_{g}-x_{g} \vec{K} ; x_{3}, \vec{k}_{3}+x_{3} \vec{q}-\vec{q}_{23}\right) .
$$

The symmetry factor of 6 includes the contribution from diagram Fig. $7\left(q_{3} q_{3} q_{2}\right)$ with the gluon emission and absorption vertices between quarks 1 and 2 interchanged.

Fig. $7\left(q_{1} q_{1} q_{2}\right)=\left(C_{F}-\frac{N_{c}+1}{2}\right) \operatorname{tr} t^{a} t^{b} t^{c} J\left(\frac{z_{2} \vec{p}_{2}-\left(1-z_{2}\right) \vec{k}_{g}}{\left(z_{2} \vec{p}_{2}-\left(1-z_{2}\right) \vec{k}_{g}\right)^{2}} \cdot \frac{z_{1} \vec{p}_{1}-\vec{k}_{g}}{\left(z_{1} \vec{p}_{1}-\vec{k}_{g}\right)^{2}}\right.$

$$
\begin{aligned}
& \left.+\frac{z_{2}\left(\vec{p}_{2}-\vec{q}_{1}\right)-\left(1-z_{2}\right) \vec{k}_{g}}{\left(z_{2}\left(\vec{p}_{2}-\vec{q}_{1}\right)-\left(1-z_{2}\right) \vec{k}_{g}\right)^{2}} \cdot \frac{z_{1}\left(\vec{p}_{1}-\vec{q}_{23}\right)-\vec{k}_{g}}{\left(z_{1}\left(\vec{p}_{1}-\vec{q}_{23}\right)-\vec{k}_{g}\right)^{2}}\right) \\
& \times \Psi_{q q q}^{*}\left(x_{1}-x_{g}, \vec{k}_{1}+x_{1} \vec{q}-\vec{q}_{23}-\vec{k}_{g}+x_{g} \vec{K}_{2} x_{2}+x_{g}, \vec{k}_{2}+x_{2} \vec{q}-\vec{q}_{1}+\vec{k}_{g}-x_{g} \vec{K} ; x_{3}, \vec{k}_{3}+x_{3} \vec{q}\right) .
\end{aligned}
$$

The symmetry factor of 6 includes the contribution from diagram Fig. $7\left(q_{2} q_{2} q_{1}\right)$ with the gluon emission and absorption vertices between quarks 1 and 2 interchanged.

Fig. $7\left(q_{2} q_{1} q_{2}\right)=\left(C_{F}-\frac{N_{c}+1}{2}\right) \operatorname{tr} t^{a} t^{c} t^{b} J\left(\frac{z_{2} \vec{p}_{2}-\left(1-z_{2}\right) \vec{k}_{g}}{\left(z_{2} \vec{p}_{2}-\left(1-z_{2}\right) \vec{k}_{g}\right)^{2}} \cdot \frac{z_{1} \vec{p}_{1}-\vec{k}_{g}}{\left(z_{1} \vec{p}_{1}-\vec{k}_{g}\right)^{2}}\right.$

$$
\begin{aligned}
& \left.+\frac{z_{2}\left(\vec{p}_{2}-\vec{q}_{13}\right)-\left(1-z_{2}\right) \vec{k}_{g}}{\left(z_{2}\left(\vec{p}_{2}-\vec{q}_{13}\right)-\left(1-z_{2}\right) \vec{k}_{g}\right)^{2}} \cdot \frac{z_{1}\left(\vec{p}_{1}-\vec{q}_{2}\right)-\vec{k}_{g}}{\left(z_{1}\left(\vec{p}_{1}-\vec{q}_{2}\right)-\vec{k}_{g}\right)^{2}}\right) \\
& \times \Psi_{q q q}^{*}\left(x_{1}-x_{g}, \vec{k}_{1}+x_{1} \vec{q}-\vec{q}_{2}-\vec{k}_{g}+x_{g} \vec{K} ; x_{2}+x_{g}, \vec{k}_{2}+x_{2} \vec{q}-\vec{q}_{13}+\vec{k}_{g}-x_{g} \vec{K} ; x_{3}, \vec{k}_{3}+x_{3} \vec{q}\right) .
\end{aligned}
$$

The symmetry factor of 6 includes the contribution from diagram Fig. $7\left(q_{1} q_{2} q_{1}\right)$ with the gluon emission and absorption vertices between quarks 1 and 2 interchanged. 
Fig. $7\left(q_{3} q_{1} q_{2}\right)=-\left(C_{F}-\frac{N_{c}+1}{2}\right)\left(\operatorname{tr} t^{a} t^{b} t^{c}+\operatorname{tr} t^{a} t^{c} t^{b}\right) J\left(\frac{z_{2} \vec{p}_{2}-\left(1-z_{2}\right) \vec{k}_{g}}{\left(z_{2} \vec{p}_{2}-\left(1-z_{2}\right) \vec{k}_{g}\right)^{2}} \cdot \frac{z_{1} \vec{p}_{1}-\vec{k}_{g}}{\left(z_{1} \vec{p}_{1}-\vec{k}_{g}\right)^{2}}\right.$

$$
\begin{aligned}
& \left.+\frac{z_{2}\left(\vec{p}_{2}-\vec{q}_{1}\right)-\left(1-z_{2}\right) \vec{k}_{g}}{\left(z_{2}\left(\vec{p}_{2}-\vec{q}_{1}\right)-\left(1-z_{2}\right) \vec{k}_{g}\right)^{2}} \cdot \frac{z_{1}\left(\vec{p}_{1}-\vec{q}_{2}\right)-\vec{k}_{g}}{\left(z_{1}\left(\vec{p}_{1}-\vec{q}_{2}\right)-\vec{k}_{g}\right)^{2}}\right) \\
& \times \Psi_{q q q}^{*}\left(x_{1}-x_{g}, \vec{k}_{1}+x_{1} \vec{q}-\vec{q}_{2}-\vec{k}_{g}+x_{g} \vec{K} ; x_{2}+x_{g}, \vec{k}_{2}+x_{2} \vec{q}-\vec{q}_{1}+\vec{k}_{g}-x_{g} \vec{K} ; x_{3}, \vec{k}_{3}+x_{3} \vec{q}-\vec{q}_{3}\right) .
\end{aligned}
$$

The symmetry factor of 6 includes the contribution from diagram Fig. $7\left(q_{3} q_{2} q_{1}\right)$ with the gluon emission and absorption vertices between quarks 1 and 2 interchanged.

Fig.7 $\left(q_{1} q_{2} q_{2}\right)=\left(C_{F}-\frac{N_{c}+1}{2}\right) \operatorname{tr} t^{a} t^{b} t^{c} J\left(\frac{z_{2} \vec{p}_{2}-\left(1-z_{2}\right) \vec{k}_{g}}{\left(z_{2} \vec{p}_{2}-\left(1-z_{2}\right) \vec{k}_{g}\right)^{2}} \cdot \frac{z_{1} \vec{p}_{1}-\vec{k}_{g}}{\left(z_{1} \vec{p}_{1}-\vec{k}_{g}\right)^{2}}\right.$

$$
\begin{aligned}
& \left.+\frac{z_{2}\left(\vec{p}_{2}-\vec{q}_{12}\right)-\left(1-z_{2}\right) \vec{k}_{g}}{\left(z_{2}\left(\vec{p}_{2}-\vec{q}_{12}\right)-\left(1-z_{2}\right) \vec{k}_{g}\right)^{2}} \cdot \frac{z_{1}\left(\vec{p}_{1}-\vec{q}_{3}\right)-\vec{k}_{g}}{\left(z_{1}\left(\vec{p}_{1}-\vec{q}_{3}\right)-\vec{k}_{g}\right)^{2}}\right) \\
& \times \Psi_{q q q}^{*}\left(x_{1}-x_{g}, \vec{k}_{1}+x_{1} \vec{q}-\vec{q}_{3}-\vec{k}_{g}+x_{g} \vec{K} ; x_{2}+x_{g}, \vec{k}_{2}+x_{2} \vec{q}-\vec{q}_{12}+\vec{k}_{g}-x_{g} \vec{K} ; x_{3}, \vec{k}_{3}+x_{3} \vec{q}\right) .
\end{aligned}
$$

The symmetry factor of 6 includes the contribution from diagram Fig. $7\left(q_{2} q_{1} q_{1}\right)$ with the gluon emission and absorption vertices between quarks 1 and 2 interchanged.

$$
\begin{aligned}
& \text { Fig. } 7\left(q_{2} q_{2} q_{2}\right)=C_{F} \operatorname{tr} t^{a} t^{b} t^{c} J\left(\frac{z_{2} \vec{p}_{2}-\left(1-z_{2}\right) \vec{k}_{g}}{\left(z_{2} \vec{p}_{2}-\left(1-z_{2}\right) \vec{k}_{g}\right)^{2}}+\frac{z_{2}\left(\vec{p}_{2}-\vec{q}\right)-\left(1-z_{2}\right) \vec{k}_{g}}{\left(z_{2}\left(\vec{p}_{2}-\vec{q}\right)-\left(1-z_{2}\right) \vec{k}_{g}\right)^{2}}\right) \cdot \frac{z_{1} \vec{p}_{1}-\vec{k}_{g}}{\left(z_{1} \vec{p}_{1}-\vec{k}_{g}\right)^{2}} \\
& \times \Psi_{q q q}^{*}\left(x_{1}-x_{g}, \vec{k}_{1}+x_{1} \vec{q}-\vec{k}_{g}+x_{g} \vec{K} ; x_{2}+x_{g}, \vec{k}_{2}+x_{2} \vec{q}-\vec{q}+\vec{k}_{g}-x_{g} \vec{K} ; x_{3}, \vec{k}_{3}+x_{3} \vec{q}\right) \text {. }
\end{aligned}
$$

The symmetry factor of 6 includes the contribution from diagram Fig. $7\left(q_{1} q_{1} q_{1}\right)$ with the gluon emission and absorption vertices between quarks 1 and 2 interchanged.

Fig.7 $\left(q_{3} q_{2} q_{2}\right)=-\left(2 C_{F}-\frac{N_{c}+1}{2}\right) \operatorname{tr} t^{a} t^{b} t^{c} J\left(\frac{z_{2} \vec{p}_{2}-\left(1-z_{2}\right) \vec{k}_{g}}{\left(z_{2} \vec{p}_{2}-\left(1-z_{2}\right) \vec{k}_{g}\right)^{2}}+\frac{z_{2}\left(\vec{p}_{2}-\vec{q}_{12}\right)-\left(1-z_{2}\right) \vec{k}_{g}}{\left(z_{2}\left(\vec{p}_{2}-\vec{q}_{12}\right)-\left(1-z_{2}\right) \vec{k}_{g}\right)^{2}}\right) \cdot \frac{z_{1} \vec{p}_{1}-\vec{k}_{g}}{\left(z_{1} \vec{p}_{1}-\vec{k}_{g}\right)^{2}}$

$$
\times \Psi_{q q q}^{*}\left(x_{1}-x_{g}, \vec{k}_{1}+x_{1} \vec{q}-\vec{k}_{g}+x_{g} \vec{K} ; x_{2}+x_{g}, \vec{k}_{2}+x_{2} \vec{q}-\vec{q}_{12}+\vec{k}_{g}-x_{g} \vec{K} ; x_{3}, \vec{k}_{3}+x_{3} \vec{q}-\vec{q}_{3}\right) .
$$

The symmetry factor of 6 includes the contribution from diagram Fig. $7\left(q_{3} q_{1} q_{1}\right)$ with the gluon emission and absorption vertices between quarks 1 and 2 interchanged.

Fig. $7\left(q_{1} q_{3} q_{2}\right)=-\left(C_{F}-\frac{N_{c}+1}{2}\right)\left(\operatorname{tr} t^{a} t^{c} t^{b}+\operatorname{tr}^{a} t^{b} t^{c}\right) J\left(\frac{z_{2} \vec{p}_{2}-\left(1-z_{2}\right) \vec{k}_{g}}{\left(z_{2} \vec{p}_{2}-\left(1-z_{2}\right) \vec{k}_{g}\right)^{2}} \cdot \frac{z_{1} \vec{p}_{1}-\vec{k}_{g}}{\left(z_{1} \vec{p}_{1}-\vec{k}_{g}\right)^{2}}\right.$

$$
\begin{aligned}
& \left.+\frac{z_{2}\left(\vec{p}_{2}-\vec{q}_{1}\right)-\left(1-z_{2}\right) \vec{k}_{g}}{\left(z_{2}\left(\vec{p}_{2}-\vec{q}_{1}\right)-\left(1-z_{2}\right) \vec{k}_{g}\right)^{2}} \cdot \frac{z_{1}\left(\vec{p}_{1}-\vec{q}_{3}\right)-\vec{k}_{g}}{\left(z_{1}\left(\vec{p}_{1}-\vec{q}_{3}\right)-\vec{k}_{g}\right)^{2}}\right) \\
& \times \Psi_{q q q}^{*}\left(x_{1}-x_{g}, \vec{k}_{1}+x_{1} \vec{q}-\vec{q}_{3}-\vec{k}_{g}+x_{g} \vec{K} ; x_{2}+x_{g}, \vec{k}_{2}+x_{2} \vec{q}-\vec{q}_{1}+\vec{k}_{g}-x_{g} \vec{K} ; x_{3}, \vec{k}_{3}+x_{3} \vec{q}-\vec{q}_{2}\right) .
\end{aligned}
$$

The symmetry factor of 6 includes the contribution from diagram Fig. $7\left(q_{2} q_{3} q_{1}\right)$ with the gluon emission and absorption vertices between quarks 1 and 2 interchanged. 
Fig.7 $\left(q_{2} q_{3} q_{2}\right)=-\left(2 C_{F}-\frac{N_{c}+1}{2}\right) \operatorname{tr} t^{a} t^{c} t^{b} J\left(\frac{z_{2} \vec{p}_{2}-\left(1-z_{2}\right) \vec{k}_{g}}{\left(z_{2} \vec{p}_{2}-\left(1-z_{2}\right) \vec{k}_{g}\right)^{2}}+\frac{z_{2}\left(\vec{p}_{2}-\vec{q}_{13}\right)-\left(1-z_{2}\right) \vec{k}_{g}}{\left(z_{2}\left(\vec{p}_{2}-\vec{q}_{13}\right)-\left(1-z_{2}\right) \vec{k}_{g}\right)^{2}} \cdot\right) \cdot \frac{z_{1} \vec{p}_{1}-\vec{k}_{g}}{\left(z_{1} \vec{p}_{1}-\vec{k}_{g}\right)^{2}}$

$$
\times \Psi_{q q q}^{*}\left(x_{1}-x_{g}, \vec{k}_{1}+x_{1} \vec{q}-\vec{k}_{g}+x_{g} \vec{K} ; x_{2}+x_{g}, \vec{k}_{2}+x_{2} \vec{q}-\vec{q}_{13}+\vec{k}_{g}-x_{g} \vec{K} ; x_{3}, \vec{k}_{3}+x_{3} \vec{q}-\vec{q}_{2}\right) .
$$

The symmetry factor of 6 includes the contribution from diagram Fig. $7\left(q_{1} q_{3} q_{1}\right)$ with the gluon emission and absorption vertices between quarks 1 and 2 interchanged.

Fig.7 $\left(q_{3} q_{3} q_{2}\right)=-\left(2 C_{F}-\frac{N_{c}+1}{2}\right) \operatorname{tr} t^{a} t^{b} t^{c} J\left(\frac{z_{2} \vec{p}_{2}-\left(1-z_{2}\right) \vec{k}_{g}}{\left(z_{2} \vec{p}_{2}-\left(1-z_{2}\right) \vec{k}_{g}\right)^{2}}+\frac{z_{2}\left(\vec{p}_{2}-\vec{q}_{1}\right)-\left(1-z_{2}\right) \vec{k}_{g}}{\left(z_{2}\left(\vec{p}_{2}-\vec{q}_{1}\right)-\left(1-z_{2}\right) \vec{k}_{g}\right)^{2}}\right) \cdot \frac{z_{1} \vec{p}_{1}-\vec{k}_{g}}{\left(z_{1} \vec{p}_{1}-\vec{k}_{g}\right)^{2}}$

$$
\times \Psi_{q q q}^{*}\left(x_{1}-x_{g}, \vec{k}_{1}+x_{1} \vec{q}-\vec{k}_{g}+x_{g} \vec{K} ; x_{2}+x_{g}, \vec{k}_{2}+x_{2} \vec{q}-\vec{q}_{1}+\vec{k}_{g}-x_{g} \vec{K} ; x_{3}, \vec{k}_{3}+x_{3} \vec{q}-\vec{q}_{23}\right) .
$$

The symmetry factor of 6 includes the contribution from diagram Fig. $7\left(q_{3} q_{3} q_{1}\right)$ with the gluon emission and absorption vertices between quarks 1 and 2 interchanged.

Fig.7 $\left(q_{1} q_{1} q_{3}\right)=-\left(2 C_{F}-\frac{N_{c}+1}{2}\right) \operatorname{tr} t^{a} t^{b} t^{c} J \frac{z_{2} \vec{p}_{2}-\left(1-z_{2}\right) \vec{k}_{g}}{\left(z_{2} \vec{p}_{2}-\left(1-z_{2}\right) \vec{k}_{g}\right)^{2}} \cdot\left(\frac{z_{1} \vec{p}_{1}-\vec{k}_{g}}{\left(z_{1} \vec{p}_{1}-\vec{k}_{g}\right)^{2}}+\frac{z_{1}\left(\vec{p}_{1}-\vec{q}_{23}\right)-\vec{k}_{g}}{\left(z_{1}\left(\vec{p}_{1}-\vec{q}_{23}\right)-\vec{k}_{g}\right)^{2}}\right)$

$$
\times \Psi_{q q q}^{*}\left(x_{1}-x_{g}, \vec{k}_{1}+x_{1} \vec{q}-\vec{q}_{23}-\vec{k}_{g}+x_{g} \vec{K} ; x_{2}+x_{g}, \vec{k}_{2}+x_{2} \vec{q}+\vec{k}_{g}-x_{g} \vec{K} ; x_{3}, \vec{k}_{3}+x_{3} \vec{q}-\vec{q}_{1}\right) .
$$

The symmetry factor of 6 includes the contribution from diagram Fig. $7\left(q_{2} q_{2} q_{3}\right)$ with the gluon emission and absorption vertices between quarks 1 and 2 interchanged.

$$
\begin{aligned}
\text { Fig.7 }\left(q_{2} q_{1} q_{3}\right)= & -\left(C_{F}-\frac{N_{c}+1}{2}\right)\left(\operatorname{tr} t^{a} t^{c} t^{b}+\operatorname{tr} t^{a} t^{b} t^{c}\right) J\left(\frac{z_{2} \vec{p}_{2}-\left(1-z_{2}\right) \vec{k}_{g}}{\left(z_{2} \vec{p}_{2}-\left(1-z_{2}\right) \vec{k}_{g}\right)^{2}} \cdot \frac{z_{1} \vec{p}_{1}-\vec{k}_{g}}{\left(z_{1} \vec{p}_{1}-\vec{k}_{g}\right)^{2}}\right. \\
& \left.+\frac{z_{2}\left(\vec{p}_{2}-\vec{q}_{3}\right)-\left(1-z_{2}\right) \vec{k}_{g}}{\left(z_{2}\left(\vec{p}_{2}-\vec{q}_{3}\right)-\left(1-z_{2}\right) \vec{k}_{g}\right)^{2}} \cdot \frac{z_{1}\left(\vec{p}_{1}-\vec{q}_{2}\right)-\vec{k}_{g}}{\left(z_{1}\left(\vec{p}_{1}-\vec{q}_{2}\right)-\vec{k}_{g}\right)^{2}}\right) \\
& \times \Psi_{q q}^{*}\left(x_{1}-x_{g}, \vec{k}_{1}+x_{1} \vec{q}-\vec{q}_{2}-\vec{k}_{g}+x_{g} \vec{K} ; x_{2}+x_{g}, \vec{k}_{2}+x_{2} \vec{q}-\vec{q}_{3}+\vec{k}_{g}-x_{g} \vec{K} ; x_{3}, \vec{k}_{3}+x_{3} \vec{q}-\vec{q}_{1}\right) .
\end{aligned}
$$

The symmetry factor of 6 includes the contribution from diagram Fig. $7\left(q_{1} q_{2} q_{3}\right)$ with the gluon emission and absorption vertices between quarks 1 and 2 interchanged.

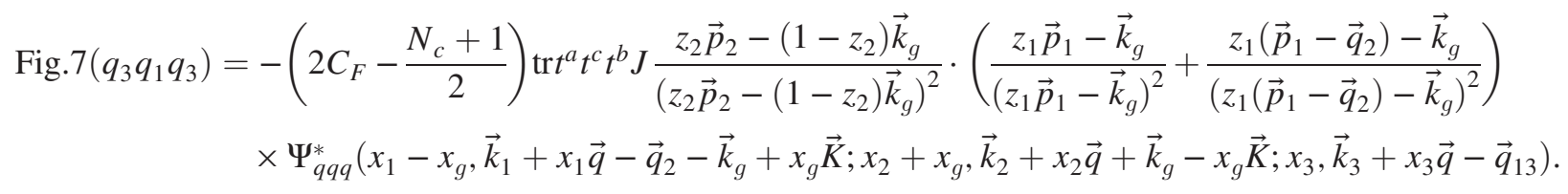

The symmetry factor of 6 includes the contribution from diagram Fig. $7\left(q_{3} q_{2} q_{3}\right)$ with the gluon emission and absorption vertices between quarks 1 and 2 interchanged.

Fig. $7\left(q_{1} q_{2} q_{3}\right)=-\left(C_{F}-\frac{N_{c}+1}{2}\right)\left(\operatorname{tr} t^{a} t^{b} t^{c}+\operatorname{tr} t^{a} t^{c} t^{b}\right) J\left(\frac{z_{2} \vec{p}_{2}-\left(1-z_{2}\right) \vec{k}_{g}}{\left(z_{2} \vec{p}_{2}-\left(1-z_{2}\right) \vec{k}_{g}\right)^{2}} \cdot \frac{z_{1} \vec{p}_{1}-\vec{k}_{g}}{\left(z_{1} \vec{p}_{1}-\vec{k}_{g}\right)^{2}}\right.$

$$
\begin{aligned}
& \left.+\frac{z_{2}\left(\vec{p}_{2}-\vec{q}_{2}\right)-\left(1-z_{2}\right) \vec{k}_{g}}{\left(z_{2}\left(\vec{p}_{2}-\vec{q}_{2}\right)-\left(1-z_{2}\right) \vec{k}_{g}\right)^{2}} \cdot \frac{z_{1}\left(\vec{p}_{1}-\vec{q}_{3}\right)-\vec{k}_{g}}{\left(z_{1}\left(\vec{p}_{1}-\vec{q}_{3}\right)-\vec{k}_{g}\right)^{2}}\right) \\
& \times \Psi_{q q q}^{*}\left(x_{1}-x_{g}, \vec{k}_{1}+x_{1} \vec{q}-\vec{q}_{3}-\vec{k}_{g}+x_{g} \vec{K} ; x_{2}+x_{g}, \vec{k}_{2}+x_{2} \vec{q}-\vec{q}_{2}+\vec{k}_{g}-x_{g} \vec{K} ; x_{3}, \vec{k}_{3}+x_{3} \vec{q}-\vec{q}_{1}\right) .
\end{aligned}
$$

The symmetry factor of 6 includes the contribution from diagram Fig. $7\left(q_{2} q_{1} q_{3}\right)$ with the gluon emission and absorption vertices between quarks 1 and 2 interchanged. 
Fig.7 $\left(q_{2} q_{2} q_{3}\right)=-\left(2 C_{F}-\frac{N_{c}+1}{2}\right) \operatorname{tr} t^{a} t^{b} t^{c} J\left(\frac{z_{2} \vec{p}_{2}-\left(1-z_{2}\right) \vec{k}_{g}}{\left(z_{2} \vec{p}_{2}-\left(1-z_{2}\right) \vec{k}_{g}\right)^{2}}+\frac{z_{2}\left(\vec{p}_{2}-\vec{q}_{23}\right)-\left(1-z_{2}\right) \vec{k}_{g}}{\left(z_{2}\left(\vec{p}_{2}-\vec{q}_{23}\right)-\left(1-z_{2}\right) \vec{k}_{g}\right)^{2}}\right) \cdot \frac{z_{1} \vec{p}_{1}-\vec{k}_{g}}{\left(z_{1} \vec{p}_{1}-\vec{k}_{g}\right)^{2}}$

$$
\times \Psi_{q q q}^{*}\left(x_{1}-x_{g}, \vec{k}_{1}+x_{1} \vec{q}-\vec{k}_{g}+x_{g} \vec{K} ; x_{2}+x_{g}, \vec{k}_{2}+x_{2} \vec{q}-\vec{q}_{23}+\vec{k}_{g}-x_{g} \vec{K} ; x_{3}, \vec{k}_{3}+x_{3} \vec{q}-\vec{q}_{1}\right) .
$$

The symmetry factor of 6 includes the contribution from diagram Fig. $7\left(q_{1} q_{1} q_{3}\right)$ with the gluon emission and absorption vertices between quarks 1 and 2 interchanged.

Fig.7 $\left(q_{3} q_{2} q_{3}\right)=-\left(2 C_{F}-\frac{N_{c}+1}{2}\right) \operatorname{tr} t^{a} t^{c} t^{b} J\left(\frac{z_{2} \vec{p}_{2}-\left(1-z_{2}\right) \vec{k}_{g}}{\left(z_{2} \vec{p}_{2}-\left(1-z_{2}\right) \vec{k}_{g}\right)^{2}}+\frac{z_{2}\left(\vec{p}_{2}-\vec{q}_{2}\right)-\left(1-z_{2}\right) \vec{k}_{g}}{\left(z_{2}\left(\vec{p}_{2}-\vec{q}_{2}\right)-\left(1-z_{2}\right) \vec{k}_{g}\right)^{2}}\right) \cdot \frac{z_{1} \vec{p}_{1}-\vec{k}_{g}}{\left(z_{1} \vec{p}_{1}-\vec{k}_{g}\right)^{2}}$

$$
\times \Psi_{q q q}^{*}\left(x_{1}-x_{g}, \vec{k}_{1}+x_{1} \vec{q}-\vec{k}_{g}+x_{g} \vec{K} ; x_{2}+x_{g}, \vec{k}_{2}+x_{2} \vec{q}-\vec{q}_{2}+\vec{k}_{g}-x_{g} \vec{K} ; x_{3}, \vec{k}_{3}+x_{3} \vec{q}-\vec{q}_{13}\right) .
$$

The symmetry factor of 6 includes the contribution from diagram Fig.7 $\left(q_{3} q_{1} q_{3}\right)$ with the gluon emission and absorption vertices between quarks 1 and 2 interchanged.

Fig.7 $\left(q_{1} q_{3} q_{3}\right)=-\left(2 C_{F}-\frac{N_{c}+1}{2}\right) \operatorname{tr} t^{a} t^{b} t^{c} J \frac{z_{2} \vec{p}_{2}-\left(1-z_{2}\right) \vec{k}_{g}}{\left(z_{2} \vec{p}_{2}-\left(1-z_{2}\right) \vec{k}_{g}\right)^{2}} \cdot\left(\frac{z_{1} \vec{p}_{1}-\vec{k}_{g}}{\left(z_{1} \vec{p}_{1}-\vec{k}_{g}\right)^{2}}+\frac{z_{1}\left(\vec{p}_{1}-\vec{q}_{3}\right)-\vec{k}_{g}}{\left(z_{1}\left(\vec{p}_{1}-\vec{q}_{3}\right)-\vec{k}_{g}\right)^{2}}\right)$

$$
\times \Psi_{q q q}^{*}\left(x_{1}-x_{g}, \vec{k}_{1}+x_{1} \vec{q}-\vec{q}_{3}-\vec{k}_{g}+x_{g} \vec{K} ; x_{2}+x_{g}, \vec{k}_{2}+x_{2} \vec{q}+\vec{k}_{g}-x_{g} \vec{K} ; x_{3}, \vec{k}_{3}+x_{3} \vec{q}-\vec{q}_{12}\right) .
$$

The symmetry factor of 6 includes the contribution from diagram Fig. $7\left(q_{2} q_{3} q_{3}\right)$ with the gluon emission and absorption vertices between quarks 1 and 2 interchanged.

Fig.7 $\left(q_{2} q_{3} q_{3}\right)=-\left(2 C_{F}-\frac{N_{c}+1}{2}\right) \operatorname{tr} t^{a} t^{b} t^{c} J\left(\frac{z_{2} \vec{p}_{2}-\left(1-z_{2}\right) \vec{k}_{g}}{\left(z_{2} \vec{p}_{2}-\left(1-z_{2}\right) \vec{k}_{g}\right)^{2}}+\frac{z_{2}\left(\vec{p}_{2}-\vec{q}_{3}\right)-\left(1-z_{2}\right) \vec{k}_{g}}{\left(z_{2}\left(\vec{p}_{2}-\vec{q}_{3}\right)-\left(1-z_{2}\right) \vec{k}_{g}\right)^{2}}\right) \cdot \frac{z_{1} \vec{p}_{1}-\vec{k}_{g}}{\left(z_{1} \vec{p}_{1}-\vec{k}_{g}\right)^{2}}$

$$
\times \Psi_{q q q}^{*}\left(x_{1}-x_{g}, \vec{k}_{1}+x_{1} \vec{q}-\vec{k}_{g}+x_{g} \vec{K} ; x_{2}+x_{g}, \vec{k}_{2}+x_{2} \vec{q}-\vec{q}_{3}+\vec{k}_{g}-x_{g} \vec{K} ; x_{3}, \vec{k}_{3}+x_{3} \vec{q}-\vec{q}_{12}\right) .
$$

The symmetry factor of 6 includes the contribution from diagram Fig.7 $\left(q_{1} q_{3} q_{1}\right)$ with the gluon emission and absorption vertices between quarks 1 and 2 interchanged.

$$
\begin{aligned}
& \text { Fig.7 }\left(q_{3} q_{3} q_{3}\right)=-2 C_{F}\left(2-N_{c}\right) \operatorname{tr} t^{a} t^{b} t^{c} J \frac{z_{2} \vec{p}_{2}-\left(1-z_{2}\right) \vec{k}_{g}}{\left(z_{2} \vec{p}_{2}-\left(1-z_{2}\right) \vec{k}_{g}\right)^{2}} \cdot \frac{z_{1} \vec{p}_{1}-\vec{k}_{g}}{\left(z_{1} \vec{p}_{1}-\vec{k}_{g}\right)^{2}} \\
& \times \Psi_{q q q}^{*}\left(x_{1}-x_{g}, \vec{k}_{1}+x_{1} \vec{q}-\vec{k}_{g}+x_{g} \vec{K} ; x_{2}+x_{g}, \vec{k}_{2}+x_{2} \vec{q}+\vec{k}_{g}-x_{g} \vec{K} ; x_{3}, \vec{k}_{3}+x_{3} \vec{q}-\vec{q}\right) .
\end{aligned}
$$

The symmetry factor of 6 includes the contribution from diagram Fig. $7\left(q_{3} q_{3} q_{3}\right)$ with the gluon emission and absorption vertices between quarks 1 and 2 interchanged.

\section{THE CORRELATOR IN IMPACT PARAMETER SPACE}

The vanishing of $\left\langle\rho^{a}\left(\vec{q}_{1}\right) \rho^{b}\left(\vec{q}_{2}\right) \rho^{c}\left(\vec{q}_{3}\right)\right\rangle$ when $\vec{q}_{1}=0$ or $\vec{q}_{3}=0$ leads to a sum rule in impact parameter space. Let us first separate $C$-odd and $C$-even contributions via

$$
G_{3}^{-}\left(\vec{q}_{1}, \vec{q}_{2}, \vec{q}_{3}\right) \sim d^{a b c}\left\langle\rho^{a}\left(\vec{q}_{1}\right) \rho^{b}\left(\vec{q}_{2}\right) \rho^{c}\left(\vec{q}_{3}\right)\right\rangle, \quad G_{3}^{+}\left(\vec{q}_{1}, \vec{q}_{2}, \vec{q}_{3}\right) \sim i f^{a b c}\left\langle\rho^{a}\left(\vec{q}_{1}\right) \rho^{b}\left(\vec{q}_{2}\right) \rho^{c}\left(\vec{q}_{3}\right)\right\rangle .
$$

Introducing the total momentum transfer $\vec{K}=-\left(\vec{q}_{1}+\vec{q}_{2}+\vec{q}_{3}\right)$, where we assume $\vec{P}=0$ for the incoming proton, and the relative momenta $\vec{\Delta}_{12}=\vec{q}_{1}-\vec{q}_{2}, \vec{\Delta}_{23}=\vec{q}_{2}-\vec{q}_{3}$, we can Fourier transform these correlators to impact parameter space:

$$
G_{3}^{ \pm}\left(\vec{b}, \vec{\Delta}_{12}, \vec{\Delta}_{23}\right)=\int \frac{\mathrm{d}^{2} K}{(2 \pi)^{2}} e^{-i \vec{b} \cdot \vec{K}} G_{3}^{ \pm}\left(\frac{2 \vec{\Delta}_{12}+\vec{\Delta}_{23}-\vec{K}}{3}, \frac{-\vec{\Delta}_{12}+\vec{\Delta}_{23}-\vec{K}}{3},-\frac{\vec{\Delta}_{12}+2 \vec{\Delta}_{23}+\vec{K}}{3}\right) .
$$

These functions satisfy the sum rule 


$$
\int \mathrm{d}^{2} b G_{3}^{ \pm}\left(\vec{b}, \vec{\Delta}_{12}, \vec{\Delta}_{23}\right)=0
$$

when $2 \vec{\Delta}_{12}=-\vec{\Delta}_{23}$ or $\vec{\Delta}_{12}=-2 \vec{\Delta}_{23}$ or $\vec{\Delta}_{12}=\vec{\Delta}_{23}=0$.

We proceed to show a numerical estimate for $G_{3}^{-}(b)$ for $\vec{\Delta}_{12}=\vec{\Delta}_{23}=0$, normalized according to $G_{3}^{-}\left(\vec{q}_{1}, \vec{q}_{2}, \vec{q}_{3}\right)=$ $4 d^{a b c}\left\langle\rho^{a}\left(\vec{q}_{1}\right) \rho^{b}\left(\vec{q}_{2}\right) \rho^{c}\left(\vec{q}_{3}\right)\right\rangle / g^{3}$. Here, $\left\langle\rho^{a}\left(\vec{q}_{1}\right) \rho^{b}\left(\vec{q}_{2}\right) \times\right.$ $\left.\rho^{c}\left(\vec{q}_{3}\right)\right\rangle$ is given by the sum of all diagrams computed above. For the numerical results we employ the "harmonic oscillator" three-quark model wave function $\Psi_{\mathrm{qqq}}\left(x_{i}, \vec{k}_{i}\right)$ by Brodsky and Schlumpf $[55,56]$ used also in Ref. [2], which assumes a Gaussian momentum distribution of quarks in two transverse dimensions, with a specific $x$-dependent width. Also, the magnitude of the NLO correction depends on the value of the coupling for which we use $\alpha_{s}=0.2$; and on the collinear regulator (see Appendix B) which we take as $0.2 \mathrm{GeV}$.

Our result is shown in Fig. 8. At $x=0.1$ the correction to the LO result [49] is numerically small; similar behavior was observed for the correlator of two color charge operators in Ref. [2]. This is an important check of the perturbative expansion about a three-quark Fock state. With decreasing $x$ the NLO correction grows substantially.

Figure 8 also shows that the $b$-dependence of $G_{3}^{-}$does not follow a positive definite 1-body "parton density distribution," or a proton thickness function, respectively, at small $b$. Rather, $n$-body quantum correlations of color charge depend nontrivially on the impact parameter, reflecting in a change of sign of $G_{3}^{-}$at $b \simeq 0.15 \mathrm{fm}$. Last, we note that the generic magnitude of $G_{3}^{-}$, for impact parameters in the perturbative region, is similar to that of the correlator of two color charge operators $G_{2}$ shown in Ref. [2]. Hence, for realistic values of the coupling there are substantial corrections to Gaussian color charge fluctuations in the proton at moderately small $x$.



FIG. 8. Numerical model estimate of the impact parameter dependence of the $C$-odd part of the correlator of three color charge operators in the proton.

\section{SUMMARY}

We have computed the diagrams for the light-cone gauge color charge correlator $\left\langle\rho^{a}\left(\vec{q}_{1}\right) \rho^{b}\left(\vec{q}_{2}\right) \rho^{c}\left(\vec{q}_{3}\right)\right\rangle$ in a proton made of three quarks and a perturbative gluon which is not required to carry a small light-cone momentum. This correlator provides the leading correction to Gaussian color charge fluctuations in the proton. It is independent of the renormalization scale since UV divergences cancel, but as the correlator of two color charge operators [1] it also exhibits logarithmic collinear and soft singularities.

These results may be used to obtain a more realistic picture of correlations in the proton at moderate $x \gtrsim 0.01$. In particular, there exist contributions where one cannot "pair up" the transverse momenta of two of the three exchanged gluons, i.e., where the three probes hit the target proton at three different impact parameters. Furthermore, our explicit expressions could be used as initial conditions (in the weak field / dilute regime) for small- $x$ evolution, in particular for impact parameter dependent evolution [57-61] with a contribution that is odd under $C$ and $P$.

The expressions for $\left\langle\rho^{a}\left(\vec{q}_{1}\right) \rho^{b}\left(\vec{q}_{2}\right) \rho^{c}\left(\vec{q}_{3}\right)\right\rangle$ have a form similar to those for $\left\langle\rho^{a}\left(\vec{q}_{1}\right) \rho^{b}\left(\vec{q}_{2}\right)\right\rangle$ obtained previously [1]. Therefore, they can be evaluated numerically using the same code developed for the two-point charge correlator [2]. We have provided first numerical estimates for the $C$-odd part $G_{3}^{-}$of $\left\langle\rho^{a}\left(\vec{q}_{1}\right) \rho^{b}\left(\vec{q}_{2}\right) \rho^{c}\left(\vec{q}_{3}\right)\right\rangle$ in Sec. III. We find that the NLO correction due to the $|q q q g\rangle$ Fock state of the proton is numerically small at $x=0.1$ but that it increases rapidly as $x \rightarrow 0.01$. Also, the dependence of the threecharge correlator on impact parameter $b$ is rather nontrivial, and its sign changes indicate $b$-dependent transitions in the nature of the quantum correlations of color charge in the proton. This result, together with published results on the $\left\langle\rho^{a} \rho^{b}\right\rangle$ correlator [2], provides guidance for phenomenological models of color charge correlations in the proton (at $x \sim 0.01-0.1$ ).

\section{ACKNOWLEDGMENTS}

The figures have been prepared with JaxoDraw [62]. A. D. thanks the U.S. Department of Energy, Office of Nuclear Physics, for support via Grant No. DESC0002307; and The City University of New York for PSC-CUNY Research Grant No. 64025-00 52. R. P. is supported by the Academy of Finland, Project No. 1322507 and by the European Research Council, Grant No. 725369. H. M. is supported by the Academy of Finland Projects No. 338263 and No. 346567, and by the European Research Council Project No. STRONG-2020 (Grant Agreement No. 824093). The content of this article does not reflect the official opinion of the European Union, and responsibility for the information and views expressed therein lies entirely with the authors. 
Computing resources from CSC-IT Center for Science in Espoo, Finland, and from the Finnish Grid and Cloud Infrastructure (persistent identifier urn:nbn:fi: research-infras-2016072533) were used in this work.

\section{APPENDIX A: THE PROTON ON THE LIGHT FRONT}

For completeness we briefly review in this appendix the Fock state description of the proton on the light front as used in the main text.

\section{Three quark Fock state of the proton}

The Fock space description of the eigenstates of the QCD Hamiltonian on the light cone has been discussed many times in the literature; see, e.g., Refs. [63-65]. Any such state, such as the proton, will involve a superposition of $n$ parton Fock states, for arbitrary $n$. However, it is well known from phenomenology that at $x \gtrsim 0.1$ the proton can be understood to first approximation in terms of a state of three massive quarks (with the appropriate flavors and spins, and in an antisymmetric color singlet state); see, e.g., Refs. [55,56]. Therefore, we consider such a phenomenological state of three massive quarks, a "light-cone constituent quark model," a reasonable point of expansion, to which we add a perturbative $|q q q g\rangle$ correction. $^{7}$

We write the light-cone state of an on-shell proton with four-momentum $P^{\mu}=\left(P^{+}, P^{-}, \vec{P}\right)$ composed of three quarks as

$$
\begin{aligned}
|P\rangle= & \frac{1}{\sqrt{N_{c} !}} \int\left[\mathrm{d} x_{i}\right] \int\left[\mathrm{d}^{2} k_{i}\right] \Psi_{q q q}\left(x_{1}, \vec{k}_{1} ; x_{2}, \vec{k}_{2} ; x_{3}, \vec{k}_{3}\right) \\
& \times \sum_{i_{1}, i_{2}, i_{3}} \epsilon_{i_{1} i_{2} i_{3}}\left|p_{1}, i_{1} ; p_{2}, i_{2} ; p_{3}, i_{3}\right\rangle|S\rangle .
\end{aligned}
$$

$N_{c}=3$ is the number of colors while $|S\rangle$ is the helicity wave function of the proton, normalized to $\langle S \mid S\rangle=1$. We have used the following compact notation:

$$
\begin{array}{r}
{\left[\mathrm{d} x_{i}\right] \equiv \frac{\mathrm{d} x_{1} \mathrm{~d} x_{2} \mathrm{~d} x_{3}}{8 x_{1} x_{2} x_{3}} \delta\left(1-x_{1}-x_{2}-x_{3}\right),} \\
{\left[\mathrm{d}^{2} k_{i}\right] \equiv \frac{\mathrm{d}^{2} k_{1} \mathrm{~d}^{2} k_{2} \mathrm{~d}^{2} k_{3}}{(2 \pi)^{6}} \delta\left(\vec{k}_{1}+\vec{k}_{2}+\vec{k}_{3}\right) .}
\end{array}
$$

The three on-shell quark momenta are specified by their light-cone momentum components $p_{i}^{+}=x_{i} P^{+}$and their

\footnotetext{
${ }^{7}$ Let us also note that the two-point color charge correlator $\left\langle\rho^{a}\left(\vec{q}_{1}\right) \rho^{b}\left(\vec{q}_{2}\right)\right\rangle$ at $x=0.1$ receives fairly small corrections from the perturbative gluon emission [2]. We take this as another confirmation for the validity (at such $x$ ) of expanding from a state of three massive quarks.
}

transverse components $\vec{p}_{i}=x_{i} \vec{P}+\vec{k}_{i}$. The quark colors are denoted as $i_{1,2,3} . \Psi_{q q q}$ is the three-quark wave function. It is symmetric under the exchange of any two of the quarks: $\Psi_{q q q}\left(x_{1}, \vec{k}_{1} ; x_{2}, \vec{k}_{2} ; x_{3}, \vec{k}_{3}\right)=$ $\Psi_{q q q}\left(x_{2}, \vec{k}_{2} ; x_{1}, \vec{k}_{1} ; x_{3}, \vec{k}_{3}\right)$, etc.

For simplicity, we will assume that the momentum space wave function $\Psi_{q q q}$ does not depend on the helicities $h_{i}=$ \pm 1 of the quarks, i.e., that the helicity wave function factorizes from the color-momentum wave function. Helicity matrix elements are given by $\left\langle h_{i}\right\rangle=0$ and $\left\langle h_{i} h_{j \neq i}\right\rangle=-\frac{1}{3}$ (see Ref. [1]).

We neglect plus momentum transfer so that $\xi=$ $\left(K^{+}-P^{+}\right) / P^{+} \rightarrow 0$. The proton state is then normalized according to

$$
\langle K \mid P\rangle=16 \pi^{3} P^{+} \delta\left(P^{+}-K^{+}\right) \delta(\vec{P}-\vec{K}) .
$$

The one-particle quark states introduced above are created by the action of the quark creation operator on the vacuum $|0\rangle$ :

$$
|p, i, h\rangle=b_{i h}^{\dagger}(p)|0\rangle .
$$

The quark creation and annihilation operators satisfy the anticommutation relation

$$
\left\{b_{j h}(k), b_{i h^{\prime}}^{\dagger}(p)\right\}=\delta_{h h^{\prime}}^{j i} 16 \pi^{3} k^{+} \delta\left(k^{+}-p^{+}\right) \delta(\vec{k}-\vec{p}),
$$

so that

$$
\left\langle k, j, h \mid p, i, h^{\prime}\right\rangle=\delta_{h h^{\prime}}^{j i} 16 \pi^{3} k^{+} \delta\left(k^{+}-p^{+}\right) \delta(\vec{k}-\vec{p}) .
$$

Similarly, the operators which create or destroy a gluon satisfy the commutation relations

$\left[a_{a \lambda}(k), a_{b \rho}^{\dagger}(p)\right]=\delta_{\lambda \rho}^{a b} 16 \pi^{3} k^{+} \delta\left(k^{+}-p^{+}\right) \delta(\vec{k}-\vec{p})$.

The normalization of the valence quark wave function is given by

$\frac{1}{2} \int\left[\mathrm{d} x_{i}\right] \int\left[\mathrm{d}^{2} k_{i}\right]\left|\Psi_{q q q}\left(x_{1}, \vec{k}_{1} ; x_{2}, \vec{k}_{2} ; x_{3}, \vec{k}_{3}\right)\right|^{2}=1$.

\section{Three quarks and a gluon}

In this section we outline the computation in light-cone perturbation theory of the three quark plus one gluon wave function, and of the virtual corrections $[1,66,67]$.

The physical incoming one-particle quark state can be written as a simultaneous perturbative and Fock state decomposition in terms of the bare states 


$$
\begin{aligned}
|q(p, h, i)\rangle= & Z_{q}^{1 / 2}\left(p^{+}\right)\left(|q(p, h, i)\rangle_{0}+\sum_{h^{\prime}, \sigma, j, a} \int \widetilde{\mathrm{d} k_{q}} \widetilde{\mathrm{d}}_{g}(2 \pi)^{3} \delta\left(p^{+}-k_{q}^{+}-k_{g}^{+}\right) \delta\left(\vec{p}-\vec{k}_{q}-\vec{k}_{g}\right) \psi_{q \rightarrow q g}\left(p ; k_{q}, k_{g}\right)\right. \\
& \left.\times\left|q\left(k_{q}, h^{\prime}, j\right) g\left(k_{g}, \sigma, a\right)\right\rangle_{0}+\cdots\right) .
\end{aligned}
$$

Here, the LCwf for $q \rightarrow q g$ splitting is denoted as $\psi_{q \rightarrow q g}$ and the Lorentz invariant measures $\widetilde{\mathrm{d}}_{q}$ and $\widetilde{\mathrm{d}}_{g}$ are defined as

$$
\int \widetilde{\mathrm{d} k} \equiv \int \frac{\mathrm{d} k^{+}}{2 k^{+}} \frac{\mathrm{d}^{2} k}{(2 \pi)^{3}} \rightarrow\left(\mu^{2}\right)^{2-D / 2} \int \frac{\mathrm{d} k^{+}}{(2 \pi) 2 k^{+}} \frac{\mathrm{d}^{D-2} k}{(2 \pi)^{D-2}} .
$$

The latter form is used to regularize ultraviolet (UV) divergences by integrating over the momenta of all particles in $D$ dimensions. Here, an arbitrary scale $\mu^{2}$ has been introduced so that the transverse integrals preserve their natural dimensions. The quark wave function renormalization coefficient $Z_{q}$ can be calculated from the normalization requirement

$$
\langle q(p, h, i) \mid q(p, h, i)\rangle={ }_{0}\langle q(p, h, i) \mid q(p, h, i)\rangle_{0}=2 p^{+}(2 \pi)^{3} \delta^{(3)}(0) .
$$

Now we replace each quark state in Eq. (A1) by the perturbative expansion in Eq. (A9). This yields

$$
\begin{aligned}
& \left|q\left(p_{1}, h_{1}, i_{1}\right) q\left(p_{2}, h_{2}, i_{2}\right) q\left(p_{3}, h_{3}, i_{3}\right)\right\rangle|S\rangle \\
& =\left[\left(1-\frac{C_{q}\left(p_{1}^{+}\right)}{2}\right)\left|q\left(p_{1}, h_{1}, i_{1}\right)\right\rangle_{0}+\sum_{h^{\prime}, \sigma, j, a} 2 g\left(t^{a}\right)_{j i_{1}} \int \frac{\widetilde{\mathrm{d}}_{g}}{2\left(p_{1}^{+}-k_{g}^{+}\right)} \hat{\psi}_{q \rightarrow q g}\left(p_{1} ; p_{1}-k_{g}, k_{g}\right)\right. \\
& \left.\quad \times\left|q\left(p_{1}-k_{g}, h^{\prime}, j\right) g\left(k_{g}, \sigma, a\right)\right\rangle_{0}+\cdots\right] \\
& \quad \otimes\left[\left(1-\frac{C_{q}\left(p_{2}^{+}\right)}{2}\right)\left|q\left(p_{2}, h_{2}, i_{2}\right)\right\rangle_{0}+\sum_{h^{\prime}, \sigma, j, a} 2 g\left(t^{a}\right)_{j i_{2}} \int \frac{\widetilde{\mathrm{d} k_{g}}}{2\left(p_{2}^{+}-k_{g}^{+}\right)} \hat{\psi}_{q \rightarrow q g}\left(p_{2} ; p_{2}-k_{g}, k_{g}\right)\right. \\
& \left.\quad \times\left|q\left(p_{2}-k_{g}, h^{\prime}, j\right) g\left(k_{g}, \sigma, a\right)\right\rangle_{0}+\cdots\right] \\
& \quad \otimes\left[\left(1-\frac{C_{q}\left(p_{3}^{+}\right)}{2}\right)\left|q\left(p_{3}, h_{3}, i_{3}\right)\right\rangle_{0}+\sum_{h^{\prime}, \sigma, j, a} 2 g\left(t^{a}\right)_{j i_{3}} \int \frac{\widetilde{\mathrm{d} k_{g}}}{2\left(p_{3}^{+}-k_{g}^{+}\right)} \hat{\psi}_{q \rightarrow q g}\left(p_{3} ; p_{3}-k_{g}, k_{g}\right)\right. \\
& \left.\quad \times\left|q\left(p_{3}-k_{g}, h^{\prime}, j\right) g\left(k_{g}, \sigma, a\right)\right\rangle_{0}+\cdots\right]|S\rangle .
\end{aligned}
$$

We have extracted the common factor $2 g\left(t^{a}\right)_{j i_{i}}$ from $\psi_{q \rightarrow q g}$ by defining $\psi_{q \rightarrow q g}\left(p_{i} ; p_{i}-k_{g}, k_{g}\right) \equiv$ $2 g\left(t^{a}\right)_{j i_{i}} \hat{\psi}_{q \rightarrow q g}\left(p_{i} ; p_{i}-k_{g}, k_{g}\right)$. The latter involves the quark helicities and the gluon polarization. Also note that $C_{q}\left(p_{i}^{+}\right) \sim$ $\mathcal{O}\left(g^{2}\right)$ while $\psi_{q \rightarrow q g} \sim \mathcal{O}(g)$, and that terms of order $\mathcal{O}\left(g^{3}\right)$ and higher must be dropped. Finally, the integration over the plus momentum of the gluon extends up to the plus momentum of the parent quark; for example, $k_{g}^{+}<p_{1}^{+}$in the first line, and so on.

We also need to add to the right-hand sice of Eq. (A12) the $\mathcal{O}\left(g^{2}\right)$ contributions where one quark emits a gluon which is then absorbed by a second (distinct) quark. For example, if the first quark emits and the second quark absorbs the gluon, that gives the contribution

$$
\begin{aligned}
& \sum_{h_{1}^{\prime}, h_{2}^{\prime}, \sigma, j, n, a} 4 g^{2}\left(t^{a}\right)_{j i_{1}}\left(t^{a}\right)_{n i_{2}} \int \frac{\tilde{\mathrm{d}} k_{g}}{2\left(p_{1}^{+}-k_{g}^{+}\right)} \hat{\psi}_{q \rightarrow q g}\left(p_{1} ; p_{1}-k_{g}, k_{g}\right) \frac{1}{2\left(p_{2}^{+}+k_{g}^{+}\right)} \hat{\psi}_{q g \rightarrow q}\left(p_{2}, k_{g} ; p_{2}+k_{g}\right) \\
& \quad \times\left|q\left(p_{1}-k_{g}, h_{1}^{\prime}, j\right) q\left(p_{2}+k_{g}, h_{2}^{\prime}, n\right)\right\rangle_{0} \otimes\left|q\left(p_{3}, h_{3}, i_{3}\right)\right\rangle_{0}|S\rangle .
\end{aligned}
$$

Here, the integration over $k_{g}^{+}$extends up to $\min \left(p_{1}^{+}, P^{+}-p_{2}^{+}\right)$. There are analogous contributions corresponding to gluon emission from quark 2 and absorption by quark 1 as well as from other pairings. 


\section{APPENDIX B: CANCELLATION OF UV DIVERGENCES}

Here, we verify the cancellation of UV divergences. We quote the following result from the appendix of Ref. [1]:

$$
\begin{aligned}
& \frac{(2 \pi)^{D-1}}{2 p_{1}^{+}} \int \frac{\widetilde{\mathrm{d}}_{g}}{2\left(p_{1}^{+}-k_{g}^{+}\right)}\left\langle S\left|\hat{\psi}_{q \rightarrow q g}\left(\vec{p}_{1} ; \vec{p}_{1}-\vec{k}_{g}, \vec{k}_{g}\right) \hat{\psi}_{q \rightarrow q g}^{*}\left(\vec{p}_{1}-\vec{l}, \vec{p}_{1}-\vec{k}_{g}-\vec{l}_{1}, \vec{k}_{g}-\vec{l}+\vec{l}_{1}\right)\right| S\right\rangle \\
& =2 \pi^{3} \frac{C_{q}\left(x_{1}\right)}{g^{2} C_{\mathrm{F}}}+2 \pi^{3} F\left(\vec{l}, \vec{l}_{1} ; \frac{x}{x_{1}}, m^{2}\right),
\end{aligned}
$$

where $\vec{l}, \vec{l}_{1}$ are $2 \mathrm{D}$ transverse momenta $\left(l^{+}=l_{1}^{+}=0\right), C_{q}(x)=1-Z_{q}(x)$ is the $\mathcal{O}\left(g^{2}\right)$ correction to the quark wave function renormalization factor, and $F$ is a UV finite function satisfying $F\left(0,0 ; x / x_{1}, m^{2}\right) \rightarrow 0$. Note that the UV divergent part does not depend on the momenta $\vec{l}, \vec{l}_{1}$. The parameter $m^{2}$ is an infrared regulator for the DGLAP collinear singularity [68-71], and $x$ is a cutoff for the soft singularity.

We begin by collecting the terms proportional to

$$
\frac{g^{3}}{8 C_{F}} \int\left[\mathrm{d} x_{i}\right] \int\left[\mathrm{d}^{2} k_{i}\right] \Psi_{q q q}\left(x_{1}, \vec{k}_{1} ; x_{2}, \vec{k}_{2} ; x_{3}, \vec{k}_{3}\right) \Psi_{q q q}^{*}\left(x_{1}, \vec{k}_{1}-\left(1-x_{1}\right) \vec{q} ; x_{2}, \vec{k}_{2}+x_{2} \vec{q} ; x_{3}, \vec{k}_{3}+x_{3} \vec{q}\right) C_{q}\left(x_{1}\right)
$$

These are Eqs. (13), (14), (15), (16), (20), (21), (22), the first term in Eq. (35) times $-C_{q}\left(x_{1}\right)$, and Eq. (36):

$$
\begin{aligned}
& 2 \frac{3}{2} i f^{a b c}+2 \frac{3}{4}\left(d^{a b c}-i f^{a b c}\right)+2 \frac{3}{4}\left(d^{a b c}+i f^{a b c}\right) \\
& +2 \frac{3}{4}\left(d^{a b c}-i f^{a b c}\right)-6 \frac{1}{4}\left(d^{a b c}+i f^{a b c}\right) \\
& -6 \frac{1}{4}\left(d^{a b c}-i f^{a b c}\right)-6 \frac{1}{4}\left(d^{a b c}+i f^{a b c}\right) \\
& -\frac{8 C_{F}}{2} \frac{1}{4}\left(d^{a b c}+i f^{a b c}\right)+4 C_{F} \frac{1}{4}\left(d^{a b c}+i f^{a b c}\right)=0 .
\end{aligned}
$$

Next, consider the diagrams which involve $\Psi_{q q q}^{*}\left(x_{1}, \vec{k}_{1}+\right.$ $\left.x_{1} \vec{q} ; x_{2}, \vec{k}_{2}+x_{2} \vec{q}-\vec{q} ; x_{3}, \vec{k}_{3}+x_{3} \vec{q}\right)$ in Eq. (B2). These are the first term in Eq. (35) times $-C_{q}\left(x_{2}\right)$ and with quarks 1 and 2 interchanged; and half of Eq. (44):

$$
-\frac{8 C_{F}}{2} \frac{1}{4}\left(d^{a b c}+i f^{a b c}\right)+2 C_{F} \cdot 2 \frac{1}{4}\left(d^{a b c}+i f^{a b c}\right)=0 .
$$

Similarly, the diagrams which involve $\Psi_{q q q}^{*}\left(x_{1}, \vec{k}_{1}+\right.$ $x_{1} \vec{q} ; x_{2}, \vec{k}_{2}+x_{2} \vec{q} ; x_{3}, \vec{k}_{3}+x_{3} \vec{q}-\vec{q}$ ) in Eq. (B2) are the first term in Eq. (35) times $-C_{q}\left(x_{3}\right)$ and with quarks 1 and 3 interchanged; and the remaining half of Eq. (44) with quarks 2 and 3 interchanged:

$$
\begin{aligned}
- & \frac{8 C_{F}}{2} \frac{1}{4}\left(d^{a b c}+i f^{a b c}\right) \\
& +2 C_{F} \cdot 2 \frac{1}{4}\left(d^{a b c}+i f^{a b c}\right)=0 .
\end{aligned}
$$

The above were diagrams where the transverse momentum of all three probe gluons flowed into one single quark line. We now consider the divergent diagrams where one of the momenta flows into a different quark line.

We begin with terms which involve $\Psi_{q q q}^{*}\left(x_{1}, \vec{k}_{1}+x_{1} \vec{q}-\right.$ $\left.\vec{q}_{12} ; x_{2}, \vec{k}_{2}+x_{2} \vec{q}-\vec{q}_{3} ; x_{3}, \vec{k}_{3}+x_{3} \vec{q}\right)$. These are Eq. (17), the fourth line in Eq. (35) times $-C_{q}\left(x_{1}\right)$, and Eq. (37):

$$
\begin{aligned}
- & 4 \frac{1}{4} \frac{3}{2}\left(d^{a b c}+i f^{a b c}\right)+\frac{8 C_{F}}{2} \frac{1}{4}\left(d^{a b c}+i f^{a b c}\right) \\
+ & 4 \frac{1}{6} \frac{1}{4}\left(d^{a b c}+i f^{a b c}\right)=0 .
\end{aligned}
$$

Now are terms which involve $\Psi_{q q q}^{*}\left(x_{1}, \vec{k}_{1}+x_{1} \vec{q}-\vec{q}_{13}\right.$; $\left.x_{2}, \vec{k}_{2}+x_{2} \vec{q}-\vec{q}_{2} ; x_{3}, \vec{k}_{3}+x_{3} \vec{q}\right)$ : Eq. (18), the third line in Eq. (35) times $-C_{q}\left(x_{1}\right)$, and Eq. (38):

$$
\begin{gathered}
-4 \frac{1}{4} \frac{3}{2}\left(d^{a b c}-i f^{a b c}\right)+\frac{8 C_{F}}{2} \frac{1}{4}\left(d^{a b c}-i f^{a b c}\right) \\
+4 \frac{1}{6} \frac{1}{4}\left(d^{a b c}-i f^{a b c}\right)=0 .
\end{gathered}
$$

Next are terms which involve $\Psi_{q q q}^{*}\left(x_{1}, \vec{k}_{1}+x_{1} \vec{q}-\vec{q}_{23}\right.$; $\left.x_{2}, \vec{k}_{2}+x_{2} \vec{q}-\vec{q}_{1} ; x_{3}, \vec{k}_{3}+x_{3} \vec{q}\right)$ : Eq. (19), the second line in Eq. (35) times $-C_{q}\left(x_{2}\right)$ and with quarks 1 and 2 interchanged, and Eq. (41):

$$
\begin{aligned}
- & 4 \frac{1}{4} \frac{3}{2}\left(d^{a b c}+i f^{a b c}\right)+\frac{8 C_{F}}{2} \frac{1}{4}\left(d^{a b c}+i f^{a b c}\right) \\
+ & 4 \frac{1}{6} \frac{1}{4}\left(d^{a b c}+i f^{a b c}\right)=0 .
\end{aligned}
$$

Terms which involve $\Psi_{q q q}^{*}\left(x_{1}, \vec{k}_{1}+x_{1} \vec{q}-\vec{q}_{1} ; x_{2}, \vec{k}_{2}+\right.$ $\left.x_{2} \vec{q}-\vec{q}_{23} ; x_{3}, \vec{k}_{3}+x_{3} \vec{q}\right)$ are the following: Eq. (29), the second line in Eq. (35) times $-C_{q}\left(x_{1}\right)$, and Eq. (39): 


$$
\begin{aligned}
- & 4 \frac{3}{2} \frac{1}{4}\left(d^{a b c}+i f^{a b c}\right)+\frac{8 C_{F}}{2} \frac{1}{4}\left(d^{a b c}+i f^{a b c}\right) \\
+ & 4 \frac{1}{6} \frac{1}{4}\left(d^{a b c}+i f^{a b c}\right)=0 .
\end{aligned}
$$

Terms which involve $\Psi_{q q q}^{*}\left(x_{1}, \vec{k}_{1}+x_{1} \vec{q}-\vec{q}_{2} ; x_{2}, \vec{k}_{2}+\right.$ $\left.x_{2} \vec{q}-\vec{q}_{13} ; x_{3}, \vec{k}_{3}+x_{3} \vec{q}\right)$ are the following: Eq. (30), the third line in Eq. (35) times $-C_{q}\left(x_{2}\right)$ with quarks 1 and 2 interchanged, and Eq. (42):

$$
\begin{aligned}
- & 4 \frac{3}{2} \frac{1}{4}\left(d^{a b c}-i f^{a b c}\right)+\frac{8 C_{F}}{2} \frac{1}{4}\left(d^{a b c}-i f^{a b c}\right) \\
+ & 4 \frac{1}{6} \frac{1}{4}\left(d^{a b c}-i f^{a b c}\right)=0 .
\end{aligned}
$$

Terms which involve $\Psi_{q q q}^{*}\left(x_{1}, \vec{k}_{1}+x_{1} \vec{q}-\vec{q}_{3} ; x_{2}, \vec{k}_{2}+\right.$ $\left.x_{2} \vec{q}-\vec{q}_{12} ; x_{3}, \vec{k}_{3}+x_{3} \vec{q}\right)$ are the following: Eq. (31), the fourth line in Eq. (35) times $-C_{q}\left(x_{2}\right)$ with quarks 1 and 2 interchanged, and Eq. (43):

$$
\begin{aligned}
- & 4 \frac{3}{2} \frac{1}{4}\left(d^{a b c}+i f^{a b c}\right)+\frac{8 C_{F}}{2} \frac{1}{4}\left(d^{a b c}+i f^{a b c}\right) \\
+ & 4 \frac{1}{6} \frac{1}{4}\left(d^{a b c}+i f^{a b c}\right)=0 .
\end{aligned}
$$

Terms which involve $\Psi_{q q q}^{*}\left(x_{1}, \vec{k}_{1}+x_{1} \vec{q} ; x_{2}, \vec{k}_{2}+x_{2} \vec{q}-\right.$ $\left.\vec{q}_{12} ; x_{3}, \vec{k}_{3}+x_{3} \vec{q}-\vec{q}_{3}\right)$ are the following: Eq. (45), and the fourth term in Eq. (35) times $-C_{q}\left(x_{3}\right)$ with quarks 1 and 3 interchanged (followed by a second interchange of quarks 2 and 3):

$$
-4 C_{F} \frac{1}{4}\left(d^{a b c}+i f^{a b c}\right)+\frac{8 C_{F}}{2} \frac{1}{4}\left(d^{a b c}+i f^{a b c}\right)=0 .
$$

Terms which involve $\Psi_{q q q}^{*}\left(x_{1}, \vec{k}_{1}+x_{1} \vec{q} ; x_{2}, \vec{k}_{2}+x_{2} \vec{q}-\right.$ $\vec{q}_{13} ; x_{3}, \vec{k}_{3}+x_{3} \vec{q}-\vec{q}_{2}$ ) are the following: Eq. (46), and the third term in Eq. (35) times $-C_{q}\left(x_{3}\right)$ with quarks 1 and 3 interchanged (followed by a second interchange of quarks 2 and 3):

$$
-4 C_{F} \frac{1}{4}\left(d^{a b c}-i f^{a b c}\right)+\frac{8 C_{F}}{2} \frac{1}{4}\left(d^{a b c}-i f^{a b c}\right)=0 .
$$

Terms which involve $\Psi_{q q q}^{*}\left(x_{1}, \vec{k}_{1}+x_{1} \vec{q} ; x_{2}, \vec{k}_{2}+x_{2} \vec{q}-\right.$ $\left.\vec{q}_{1} ; x_{3}, \vec{k}_{3}+x_{3} \vec{q}-\vec{q}_{23}\right)$ are the following: Eq. (47), and the second term in Eq. (35) times $-C_{q}\left(x_{3}\right)$ with quarks 1 and 3 interchanged (followed by a second interchange of quarks 2 and 3):

$$
-4 C_{F} \frac{1}{4}\left(d^{a b c}+i f^{a b c}\right)+\frac{8 C_{F}}{2} \frac{1}{4}\left(d^{a b c}+i f^{a b c}\right)=0 .
$$

The last structure to be checked corresponds to the diagrams where each $\vec{q}_{i}$ flows into a different quark line. Terms which involve $\Psi_{q q q}^{*}\left(x_{1}, \vec{k}_{1}+x_{1} \vec{q}-\vec{q}_{1} ; x_{2}, \vec{k}_{2}+\right.$ $x_{2} \vec{q}-\vec{q}_{2} ; x_{3}, \vec{k}_{3}+x_{3} \vec{q}-\vec{q}_{3}$ ) are the following: Eq. (32), the fifth line in Eq. (35) times $-C_{q}\left(x_{1}\right)$, and Eq. (40):

$$
4 \frac{3}{2} \frac{1}{2} d^{a b c}-\frac{8 C_{F}}{2} \frac{1}{2} d^{a b c}-4 \frac{1}{6} \frac{1}{2} d^{a b c}=0 .
$$

Terms which involve $\Psi_{q q q}^{*}\left(x_{1}, \vec{k}_{1}+x_{1} \vec{q}-\vec{q}_{2} ; x_{2}, \vec{k}_{2}+\right.$ $x_{2} \vec{q}-\vec{q}_{1} ; x_{3}, \vec{k}_{3}+x_{3} \vec{q}-\vec{q}_{3}$ ) are the following: Eq. (33), the fifth line in Eq. (35) times $-C_{q}\left(x_{2}\right)$ with quarks 1 and 2 interchanged, and Eq. (48):

$$
4 \frac{3}{2} \frac{1}{2} d^{a b c}-\frac{8 C_{F}}{2} \frac{1}{2} d^{a b c}-4 \frac{1}{6} \frac{1}{2} d^{a b c}=0 .
$$

Terms which involve $\Psi_{q q q}^{*}\left(x_{1}, \vec{k}_{1}+x_{1} \vec{q}-\vec{q}_{3} ; x_{2}, \vec{k}_{2}+\right.$ $x_{2} \vec{q}-\vec{q}_{1} ; x_{3}, \vec{k}_{3}+x_{3} \vec{q}-\vec{q}_{2}$ ) are the following: Eq. (34), the fifth line in Eq. (35) times $-C_{q}\left(x_{3}\right)$ with quarks 1 and 3 interchanged, and Eq. (49):

$$
4 \frac{3}{2} \frac{1}{2} d^{a b c}-\frac{8 C_{F}}{2} \frac{1}{2} d^{a b c}-4 \frac{1}{6} \frac{1}{2} d^{a b c}=0 .
$$

\section{APPENDIX C: WARD IDENTITY}

In this section we verify that $\left\langle\rho^{a}\left(\vec{q}_{1}\right) \rho^{b}\left(\vec{q}_{2}\right) \rho^{c}\left(\vec{q}_{3}\right)\right\rangle$ vanishes when $\vec{q}_{1} \rightarrow 0$ or $\vec{q}_{3} \rightarrow 0$ [72,73]; the first and last of the three gluon probes couple to a color singlet proton state and must not have infinite transverse wave length. On the other hand, this correlator need not vanish when $\vec{q}_{2} \rightarrow 0$ at finite $\vec{q}_{1,3}$ since the second gluon probe in that case does not couple to a color singlet. It is straightforward to confirm that the expectation value of $\rho^{a}\left(\vec{q}_{1}\right) \rho^{b}\left(\vec{q}_{2}\right) \rho^{c}\left(\vec{q}_{3}\right)$ between threequark states at $\mathcal{O}\left(g^{3}\right)$ written in Eq. (35) does indeed vanish when either $\vec{q}_{1}$ or $\vec{q}_{3} \rightarrow 0$ (see also Refs. [35,36,49]). In what follows we consider the correction at $\mathcal{O}\left(g^{5}\right)$. We first show that the finite part of the UV divergent diagrams cancel when either $\vec{q}_{1}$ or $\vec{q}_{3} \rightarrow 0$. After this we repeat the same exercise and verify that the sum of the remaining finite diagrams also cancel when either $\vec{q}_{1}$ or $\vec{q}_{3} \rightarrow 0$.

\section{UV divergent diagrams}

First, we verify that the subset of UV divergent diagrams satisfies the Ward identity, i.e., that their finite parts cancel when $\vec{q}_{1} \rightarrow 0$ or $\vec{q}_{3} \rightarrow 0$. We only demonstrate here the case $\vec{q}_{3} \rightarrow 0$ but we have checked the symmetry of these diagrams under $\vec{q}_{1} \leftrightarrow \vec{q}_{3}$. For the purpose of more compact expressions we will split off the "prefactor" 
$\frac{2 g^{5}}{3 \cdot 16 \pi^{3}} \cdot 2 \pi^{3} \int\left[\mathrm{d} x_{i}\right] \int\left[\mathrm{d}^{2} k_{i}\right] \Psi_{q q q}\left(x_{1}, \vec{k}_{1} ; x_{2}, \vec{k}_{2} ; x_{3}, \vec{k}_{3}\right)$

from the following expressions.
First, we collect all the terms from the UV divergent diagrams which involve the structure $\Psi_{q q q}\left(x_{1}, \vec{k}_{1}-\right.$ $\left.\left(1-x_{1}\right) \vec{q}_{12} ; x_{2}, \vec{k}_{2}+x_{2} \vec{q}_{12} ; x_{3}, \vec{k}_{3}+x_{3} \vec{q}_{12}\right)$. These are given by Eqs. (13), (14), (15), (16), (17), (20), (21), (22), (36), (37):

$$
\begin{aligned}
& +3 \cdot \frac{N_{c}}{2} i f^{a b c} \cdot F\left(\vec{q}_{12}, 0 ; \alpha_{1}, m^{2}\right)+3 \cdot \frac{N_{c}}{4}\left(d^{a b c}-i f^{a b c}\right) \cdot F\left(\vec{q}_{12}, 0 ; \alpha_{1}, m^{2}\right) \\
& +3 \cdot \frac{N_{c}}{4}\left(d^{a b c}+i f^{a b c}\right) \cdot F\left(\vec{q}_{12}, \vec{q}_{2} ; \alpha_{1}, m^{2}\right)+3 \cdot \frac{N_{c}}{4}\left(d^{a b c}-i f^{a b c}\right) \cdot F\left(\vec{q}_{12}, \vec{q}_{1} ; \alpha_{1}, m^{2}\right) \\
& +6 \cdot \frac{N_{c}}{2 \cdot 4}\left(d^{a b c}+i f^{a b c}\right) \cdot F\left(\vec{q}_{12}, 0 ; \alpha_{1}, m^{2}\right)-3 \cdot \frac{N_{c}}{4}\left(d^{a b c}+i f^{a b c}\right) \cdot F\left(\vec{q}_{12}, \vec{q}_{2} ; \alpha_{1}, m^{2}\right) \\
& +3 \cdot \frac{N_{c}}{4}\left(d^{a b c}-i f^{a b c}\right) \cdot F\left(\vec{q}_{12}, \vec{q}_{1} ; \alpha_{1}, m^{2}\right)-3 \cdot \frac{N_{c}}{4}\left(d^{a b c}+i f^{a b c}\right) \cdot F\left(\vec{q}_{12}, \vec{q}_{12} ; \alpha_{1}, m^{2}\right) \\
& +3 \cdot \frac{2 C_{\mathrm{F}}}{4}\left(d^{a b c}+i f^{a b c}\right) \cdot F\left(\vec{q}_{12}, \vec{q}_{12} ; \alpha_{1}, m^{2}\right)+6 \cdot \frac{1}{2 N_{c} \cdot 4}\left(d^{a b c}+i f^{a b c}\right) \cdot F\left(\vec{q}_{12}, \vec{q}_{12} ; \alpha_{1}, m^{2}\right)=0
\end{aligned}
$$

Next, we collect terms which involve the structure $\Psi_{q q q}\left(x_{1}, \vec{k}_{1}+x_{1} \vec{q}_{12}-\vec{q}_{1} ; x_{2}, \vec{k}_{2}+x_{2} \vec{q}_{12}-\vec{q}_{2} ; x_{3}, \vec{k}_{3}+x_{3} \vec{q}_{12}\right)$. These are given by Eqs. (18), (29), (32), (38), (39), (40):

$$
\begin{aligned}
& -6 \cdot \frac{N_{c}}{2 \cdot 4}\left(d^{a b c}-i f^{a b c}\right) \cdot F\left(\vec{q}_{1}, 0 ; \alpha_{1}, m^{2}\right)-6 \cdot \frac{N_{c}}{2 \cdot 4}\left(d^{a b c}+i f^{a b c}\right) \cdot F\left(\vec{q}_{1}, 0 ; \alpha_{1}, m^{2}\right) \\
& -6 \cdot \frac{N_{c}}{2 \cdot 4}\left[\left(d^{a b c}+i f^{a b c}\right)+\left(d^{a b c}-i f^{a b c}\right)\right] \cdot F\left(\vec{q}_{1}, 0 ; \alpha_{1}, m^{2}\right) \\
& +6 \cdot \frac{1}{2 N_{c} \cdot 4}\left(d^{a b c}-i f^{a b c}\right) \cdot F\left(\vec{q}_{1}, \vec{q}_{1} ; \alpha_{1}, m^{2}\right)+6 \cdot \frac{1}{2 N_{c} \cdot 4}\left(d^{a b c}+i f^{a b c}\right) \cdot F\left(\vec{q}_{1}, \vec{q}_{1} ; \alpha_{1}, m^{2}\right) \\
& -6 \cdot \frac{1}{2 N_{c} \cdot 4}\left[\left(d^{a b c}+i f^{a b c}\right)+\left(d^{a b c}-i f^{a b c}\right)\right] \cdot F\left(\vec{q}_{1}, \vec{q}_{1} ; \alpha_{1}, m^{2}\right)=0 .
\end{aligned}
$$

Similarly, the diagrams which involve the structure $\Psi_{q q q}\left(x_{1}, \vec{k}_{1}+x_{1} \vec{q}_{12}-\vec{q}_{2} ; x_{2}, \vec{k}_{2}+x_{2} \vec{q}_{12}-\vec{q}_{1} ; x_{3}, \vec{k}_{3}+x_{3} \vec{q}_{12}\right)$. These are given by Eqs. (19), (30), (33), (41), (42), (48):

$$
\begin{aligned}
- & 6 \frac{N_{c}}{2 \cdot 4}\left(d^{a b c}+i f^{a b c}\right) \cdot F\left(\vec{q}_{2}, 0 ; \alpha_{1}, m^{2}\right)-6 \cdot \frac{N_{c}}{2 \cdot 4}\left(d^{a b c}-i f^{a b c}\right) \cdot F\left(\vec{q}_{2}, 0 ; \alpha_{1}, m^{2}\right) \\
& +6 \cdot \frac{N_{c}}{2 \cdot 4}\left[\left(d^{a b c}+i f^{a b c}\right)+\left(d^{a b c}-i f^{a b c}\right)\right] \cdot F\left(\vec{q}_{2}, 0 ; \alpha_{1}, m^{2}\right) \\
& +6 \cdot \frac{1}{2 N_{c} \cdot 4}\left(d^{a b c}+i f^{a b c}\right) \cdot F\left(\vec{q}_{2}, \vec{q}_{2} ; \alpha_{1}, m^{2}\right)+6 \cdot \frac{1}{2 N_{c} \cdot 4}\left(d^{a b c}-i f^{a b c}\right) \cdot F\left(\vec{q}_{2}, \vec{q}_{2} ; \alpha_{1}, m^{2}\right) \\
& -6 \cdot \frac{1}{2 N_{c} \cdot 4}\left[\left(d^{a b c}+i f^{a b c}\right)+\left(d^{a b c}-i f^{a b c}\right)\right] \cdot F\left(\vec{q}_{2}, \vec{q}_{2} ; \alpha_{1}, m^{2}\right)=0 .
\end{aligned}
$$

Finally, all the other UV divergent diagrams (in the $\vec{q}_{3} \rightarrow 0$ case) are proportional to the finite function $F\left(0,0 ; x / x_{1}, m^{2}\right)$, which is zero.

\section{Finite diagrams}

In this section we verify that all the UV finite diagrams satisfy the Ward identity. As in the UV divergent case, the sum of all finite diagrams should cancel when $\vec{q}_{1} \rightarrow 0$ or $\vec{q}_{3} \rightarrow 0$. Here we only show details for the $\vec{q}_{3} \rightarrow 0$ case, but we have also checked that the case $\vec{q}_{1} \rightarrow 0$ satisfies the Ward identity. 
First, we collect all the terms from the UV finite diagrams which involve the structure

$$
\begin{aligned}
\vec{I} & \cdot \frac{z_{2} \vec{p}_{2}-\left(1-z_{2}\right)\left(\vec{k}_{g}-\vec{q}_{12}\right)}{\left(z_{2} \vec{p}_{2}-\left(1-z_{2}\right)\left(\vec{k}_{g}-\vec{q}_{12}\right)\right)^{2}} \\
& \times \Psi_{q q q}^{*}\left(x_{1}-x_{g}, \vec{k}_{1}+x_{1} \vec{q}_{12}-\vec{k}_{g}+x_{g} \vec{K} ; x_{2}+x_{g}, \vec{k}_{2}-\left(1-x_{2}\right) \vec{q}_{12}+\vec{k}_{g}-x_{g} \vec{K} ; x_{3}, \vec{k}_{3}+x_{3} \vec{q}_{12}\right) .
\end{aligned}
$$

These are given by Eqs. (51), (52), (55), (58):

$$
-6 \cdot \frac{1}{2} \operatorname{tr} T^{a} T^{b} T^{c}-6 \cdot \frac{1}{4} \operatorname{tr}\left(T^{a} T^{b} D^{c}-T^{a} T^{b} T^{c}\right)-6 \cdot \frac{1}{4} \operatorname{tr}\left(T^{a} T^{b} D^{c}-T^{a} T^{b} T^{c}\right)+6 \cdot \frac{1}{2} \operatorname{tr} T^{a} T^{b} D^{c}=0 .
$$

Next, we collect terms which involve the structure

$$
\begin{aligned}
\vec{I} & \cdot \frac{z_{2} \vec{p}_{2}-\left(1-z_{2}\right)\left(\vec{k}_{g}-\vec{q}_{1}\right)}{\left(z_{2} \vec{p}_{2}-\left(1-z_{2}\right)\left(\vec{k}_{g}-\vec{q}_{1}\right)\right)^{2}} \\
& \times \Psi_{q q q}^{*}\left(x_{1}-x_{g}, \vec{k}_{1}+x_{1} \vec{q}_{12}-\vec{q}_{2}-\vec{k}_{g}+x_{g} \vec{K} ; x_{2}+x_{g}, \vec{k}_{2}+x_{2} \vec{q}_{12}-\vec{q}_{1}+\vec{k}_{g}-x_{g} \vec{K} ; x_{3}, \vec{k}_{3}+x_{3} \vec{q}_{12}\right)
\end{aligned}
$$

or

$$
\begin{aligned}
\vec{I} & \cdot \frac{z_{2} \vec{p}_{2}-\left(1-z_{2}\right)\left(\vec{k}_{g}-\vec{q}_{2}\right)}{\left(z_{2} \vec{p}_{2}-\left(1-z_{2}\right)\left(\vec{k}_{g}-\vec{q}_{2}\right)\right)^{2}} \\
& \quad \times \Psi_{q q q}^{*}\left(x_{1}-x_{g}, \vec{k}_{1}+x_{1} \vec{q}_{12}-\vec{q}_{1}-\vec{k}_{g}+x_{g} \vec{K} ; x_{2}+x_{g}, \vec{k}_{2}+x_{2} \vec{q}_{12}-\vec{q}_{2}+\vec{k}_{g}-x_{g} \vec{K} ; x_{3}, \vec{k}_{3}+x_{3} \vec{q}_{12}\right) .
\end{aligned}
$$

Terms that involve the structure (C7) are given by Eqs. (53), (61), (64), (73):

$$
\begin{aligned}
-6 & \cdot \frac{1}{4} \operatorname{tr}\left(T^{a} T^{c} D^{b}-T^{a} T^{c} T^{b}\right)+6 \cdot \frac{N_{c}}{2} \operatorname{tr} t^{a} t^{b} t^{c}+6 \cdot\left(-\frac{1}{4}\left(T^{a}\right)_{b c}+\frac{N_{c}}{2} \operatorname{tr} t^{a} t^{c} t^{b}\right) \\
+ & 6 \cdot\left(-\frac{1}{4}\left(T^{a}\right)_{c b}-N_{c} \operatorname{tr} t^{a} t^{c} t^{b}+\frac{1}{4} \operatorname{tr} T^{c} T^{a}\left(D^{b}-T^{b}\right)\right) .
\end{aligned}
$$

Here the color factors can be simplified by noting that

$$
\operatorname{tr} T^{a} T^{b} D^{c}=\frac{N_{c}}{2} d^{a b c}, \quad \operatorname{tr} T^{a} T^{b} T^{c}=\frac{i N_{c}}{2} f^{a b c}, \quad \operatorname{tr} t^{a} t^{b} t^{c}=\frac{1}{4}\left(d^{a b c}+i f^{a b c}\right),
$$

where $N_{\mathrm{c}}=3$ and the structure constants $d^{a b c}$ and $f^{a b c}$ are totally symmetric and antisymmetric, respectively. This gives

$$
\begin{aligned}
-6 & \cdot \frac{1}{4}\left(\frac{N_{c}}{2} d^{a b c}+\frac{i N_{c}}{2} f^{a b c}\right)+6 \cdot \frac{1}{4}\left(\frac{N_{c}}{2} d^{a b c}+\frac{i N_{c}}{2} f^{a b c}\right) \\
& +6 \cdot \frac{1}{4}\left(-\left(T^{a}\right)_{b c}+\left(\frac{N_{c}}{2} d^{a b c}-\frac{i N_{c}}{2} f^{a b c}\right)\right)+6 \cdot \frac{1}{4}\left(+\left(T^{a}\right)_{b c}-\left(\frac{N_{c}}{2} d^{a b c}-\frac{i N_{c}}{2} f^{a b c}\right)\right)=0 .
\end{aligned}
$$

Similarly, terms which involve the structure (C8) are given by Eqs. (54), (62), (65), (74):

$$
\begin{aligned}
& -6 \cdot \frac{1}{4} \operatorname{tr}\left(T^{b} T^{c} D^{a}-T^{a} T^{b} T^{c}\right)+6 \cdot \frac{N_{c}}{2} \operatorname{tr} t^{a} t^{c} t^{b}+6 \cdot\left(+\frac{1}{4}\left(T^{b}\right)_{c a}+\frac{N_{c}}{2} \operatorname{tr} t^{a} t^{b} t^{c}\right) \\
& +6 \cdot\left(-\frac{1}{4}\left(T^{b}\right)_{c a}-N_{c} \operatorname{tr} t^{a} t^{b} t^{c}+\frac{1}{4} \operatorname{tr} T^{c} T^{b}\left(D^{a}-T^{a}\right)\right)=0 .
\end{aligned}
$$


Let us then collect all the terms which involve the structure

$$
\begin{aligned}
\vec{I} & \cdot \frac{z_{2}\left(\vec{p}_{2}-\vec{q}_{2}\right)-\left(1-z_{2}\right)\left(\vec{k}_{g}-\vec{q}_{1}\right)}{\left(z_{2}\left(\vec{p}_{2}-\vec{q}_{2}\right)-\left(1-z_{2}\right)\left(\vec{k}_{g}-\vec{q}_{1}\right)\right)^{2}} \\
& \times \Psi_{q q q}^{*}\left(x_{1}-x_{g}, \vec{k}_{1}+x_{1} \vec{q}_{12}-\vec{k}_{g}+x_{g} \vec{K} ; x_{2}+x_{g}, \vec{k}_{2}-\left(1-x_{2}\right) \vec{q}_{12}+\vec{k}_{g}-x_{g} \vec{K} ; x_{3}, \vec{k}_{3}+x_{3} \vec{q}_{12}\right)
\end{aligned}
$$

or

$$
\begin{aligned}
\vec{I} & \cdot \frac{z_{2}\left(\vec{p}_{2}-\vec{q}_{1}\right)-\left(1-z_{2}\right)\left(\vec{k}_{g}-\vec{q}_{2}\right)}{\left(z_{2}\left(\vec{p}_{2}-\vec{q}_{1}\right)-\left(1-z_{2}\right)\left(\vec{k}_{g}-\vec{q}_{2}\right)\right)^{2}} \\
& \times \Psi_{q q q}^{*}\left(x_{1}-x_{g}, \vec{k}_{1}+x_{1} \vec{q}_{12}-\vec{k}_{g}+x_{g} \vec{K} ; x_{2}+x_{g}, \vec{k}_{2}-\left(1-x_{2}\right) \vec{q}_{12}+\vec{k}_{g}-x_{g} \vec{K} ; x_{3}, \vec{k}_{3}+x_{3} \vec{q}_{12}\right) .
\end{aligned}
$$

Terms that involve the structure in (C13) are given by Eqs. (56), (67), (70), (79):

$$
\begin{aligned}
& -6 \cdot \frac{1}{4} \operatorname{tr}\left(T^{a} T^{c} D^{b}-T^{a} T^{c} T^{b}\right)+6 \cdot\left(+\frac{1}{4}\left(T^{a}\right)_{b c}+\frac{N_{c}}{2} \operatorname{tr} t^{a} t^{b} t^{c}\right)+6 \cdot \frac{N_{c}}{2} \operatorname{tr} t^{a} t^{b} t^{c} \\
& +6 \cdot\left(-\frac{1}{4}\left(T^{a}\right)_{b c}-\frac{N_{c}}{4}\left(D^{a}\right)_{b c}+\frac{1}{4} \operatorname{tr} T^{a}\left(D^{b}-T^{b}\right) T^{c}\right)=0 .
\end{aligned}
$$

Terms that involve the structure in (C14) are given by Eqs. (57), (68), (71), (80):

$$
\begin{aligned}
& -6 \cdot \frac{1}{4} \operatorname{tr}\left(T^{a} T^{b} D^{c}-T^{a} T^{b} T^{c}\right)+6 \cdot\left(+\frac{1}{4}\left(T^{b}\right)_{a c}+\frac{N_{c}}{2} \operatorname{tr} t^{a} t^{c} t^{b}\right)+6 \cdot \frac{N_{c}}{2} \operatorname{tr} t^{b} t^{a} t^{c} \\
& +6 \cdot\left(-\frac{1}{4}\left(T^{b}\right)_{a c}-\frac{N_{c}}{4}\left(D^{b}\right)_{a c}+\frac{1}{4} \operatorname{tr} T^{b}\left(D^{a}-T^{a}\right) T^{c}\right)=0 .
\end{aligned}
$$

We also have diagrams which involve the structure

$$
\begin{aligned}
\vec{I} & \cdot \frac{z_{2}\left(\vec{p}_{2}-\vec{q}_{12}\right)-\left(1-z_{2}\right) \vec{k}_{g}}{\left(z_{2}\left(\vec{p}_{2}-\vec{q}_{12}\right)-\left(1-z_{2}\right) \vec{k}_{g}\right)^{2}} \\
& \times \Psi_{q q q}^{*}\left(x_{1}-x_{g}, \vec{k}_{1}+x_{1} \vec{q}_{12}-\vec{k}_{g}+x_{g} \vec{K} ; x_{2}+x_{g}, \vec{k}_{2}-\left(1-x_{2}\right) \vec{q}_{12}+\vec{k}_{g}-x_{g} \vec{K} ; x_{3}, \vec{k}_{3}+x_{3} \vec{q}_{12}\right) .
\end{aligned}
$$

These diagrams are given by Eqs. (72), (100), (101), (102):

$$
+6 \cdot \frac{N_{c}}{2} \operatorname{tr} t^{a} t^{b} t^{c}-6 \cdot\left(C_{\mathrm{F}}-\frac{1}{2}\right) \operatorname{tr} t^{a} t^{b} t^{c}-6 \cdot C_{\mathrm{F}} \operatorname{tr} t^{a} t^{b} t^{c}+6 \cdot\left(2 C_{\mathrm{F}}-\frac{1}{2}-\frac{N_{c}}{2}\right) \operatorname{tr} t^{a} t^{b} t^{c}=0 .
$$

We then consider terms that involve the structure

$$
\begin{aligned}
\vec{I} & \cdot \frac{z_{2} \vec{p}_{2}-\left(1-z_{2}\right)\left(\vec{k}_{g}-\vec{q}_{1}\right)}{\left(z_{2} \vec{p}_{2}-\left(1-z_{2}\right)\left(\vec{k}_{g}-\vec{q}_{1}\right)\right)^{2}} \\
& \times \Psi_{q q q}^{*}\left(x_{1}-x_{g}, \vec{k}_{1}+x_{1} \vec{q}_{12}-\vec{k}_{g}+x_{g} \vec{K} ; x_{2}+x_{g}, \vec{k}_{2}+x_{2} \vec{q}_{12}-\vec{q}_{1}+\vec{k}_{g}-x_{g} \vec{K} ; x_{3}, \vec{k}_{3}+x_{3} \vec{q}_{12}-\vec{q}_{2}\right)
\end{aligned}
$$

or

$$
\begin{aligned}
\vec{I} & \cdot \frac{z_{2} \vec{p}_{2}-\left(1-z_{2}\right)\left(\vec{k}_{g}-\vec{q}_{2}\right)}{\left(z_{2} \vec{p}_{2}-\left(1-z_{2}\right)\left(\vec{k}_{g}-\vec{q}_{2}\right)\right)^{2}} \\
& \quad \times \Psi_{q q q}^{*}\left(x_{1}-x_{g}, \vec{k}_{1}+x_{1} \vec{q}_{12}-\vec{k}_{g}+x_{g} \vec{K} ; x_{2}+x_{g}, \vec{k}_{2}+x_{2} \vec{q}_{12}-\vec{q}_{2}+\vec{k}_{g}-x_{g} \vec{K} ; x_{3}, \vec{k}_{3}+x_{3} \vec{q}_{12}-\vec{q}_{1}\right) .
\end{aligned}
$$


Terms that involve the structure (C19) are given by Eqs. (59), (76), (82):

$$
\begin{aligned}
& +6 \cdot \frac{1}{2} \operatorname{tr} T^{a} T^{c} D^{b}+6 \cdot\left(-\frac{1}{4}\left(T^{a}\right)_{b c}-N_{c} \operatorname{tr} t^{a} t^{b} t^{c}+\frac{1}{4} \operatorname{tr} T^{b} T^{a}\left(D^{c}-T^{c}\right)\right) \\
& +6 \cdot\left(+\frac{1}{4}\left(T^{a}\right)_{b c}-\frac{N_{c}}{4}\left(D^{a}\right)_{b c}+\frac{1}{4} \operatorname{tr} T^{a}\left(D^{c}-T^{c}\right) T^{b}\right)=0 .
\end{aligned}
$$

Similarly, terms that involve the structure (C20) are given by Eqs. (60), (77), (83):

$$
\begin{aligned}
& +6 \cdot \frac{1}{2} \operatorname{tr} T^{a} T^{b} D^{c}+6 \cdot\left(-\frac{1}{4}\left(T^{b}\right)_{a c}-N_{c} \operatorname{tr} t^{b} t^{a} t^{c}+\frac{1}{4} \operatorname{tr} T^{a} T^{b}\left(D^{c}-T^{c}\right)\right) \\
& +6 \cdot\left(+\frac{1}{4}\left(T^{b}\right)_{a c}-\frac{N_{\mathrm{c}}}{4}\left(D^{b}\right)_{a c}+\frac{1}{4} \operatorname{tr} T^{b}\left(D^{c}-T^{c}\right) T^{a}\right)=0 .
\end{aligned}
$$

A similar set of diagrams are given by terms that involve the structure

$$
\begin{aligned}
\vec{I} & \cdot \frac{z_{2}\left(\vec{p}_{2}-\vec{q}_{1}\right)-\left(1-z_{2}\right) \vec{k}_{g}}{\left(z_{2}\left(\vec{p}_{2}-\vec{q}_{1}\right)-\left(1-z_{2}\right) \vec{k}_{g}\right)^{2}} \\
& \quad \times \Psi_{q q q}^{*}\left(x_{1}-x_{g}, \vec{k}_{1}+x_{1} \vec{q}_{12}-\vec{k}_{g}+x_{g} \vec{K}-\vec{q}_{2} ; x_{2}+x_{g}, \vec{k}_{2}+x_{2} \vec{q}_{12}-\vec{q}_{1}+\vec{k}_{g}-x_{g} \vec{K} ; x_{3}, \vec{k}_{3}+x_{3} \vec{q}_{12}\right)
\end{aligned}
$$

or

$$
\begin{aligned}
\vec{I} & \cdot \frac{z_{2}\left(\vec{p}_{2}-\vec{q}_{2}\right)-\left(1-z_{2}\right) \vec{k}_{g}}{\left(z_{2}\left(\vec{p}_{2}-\vec{q}_{2}\right)-\left(1-z_{2}\right) \vec{k}_{g}\right)^{2}} \\
& \times \Psi_{q q q}^{*}\left(x_{1}-x_{g}, \vec{k}_{1}+x_{1} \vec{q}_{12}-\vec{k}_{g}+x_{g} \vec{K}-\vec{q}_{1} ; x_{2}+x_{g}, \vec{k}_{2}+x_{2} \vec{q}_{12}-\vec{q}_{2}+\vec{k}_{g}-x_{g} \vec{K} ; x_{3}, \vec{k}_{3}+x_{3} \vec{q}_{12}\right) .
\end{aligned}
$$

Terms that involve the structure (C23) are given by Eqs. (69), (97), (98), (99):

$$
\begin{aligned}
& +6 \cdot\left(\frac{1}{4}\left(T^{c}\right)_{a b}+\frac{N_{c}}{2} \operatorname{tr} t^{a} t^{b} t^{c}\right)-6 \cdot\left(C_{\mathrm{F}}-\frac{1}{2}\right) \operatorname{tr} t^{a} t^{b} t^{c}-6 \cdot\left(C_{\mathrm{F}}-\frac{1}{2}\right) \operatorname{tr} t^{a} t^{c} t^{b} \\
& +6 \cdot\left(\left(C_{\mathrm{F}}-\frac{N_{c}}{2}\right) \operatorname{tr} t^{a} t^{b} t^{c}+\left(C_{\mathrm{F}}-1\right) \operatorname{tr} t^{a} t^{c} t^{b}\right)=0 .
\end{aligned}
$$

Terms that involve the structure (C24) are given by Eqs. (66), (91), (92), (93):

$$
\begin{aligned}
& +6 \cdot\left(\frac{1}{4}\left(T^{c}\right)_{b a}+\frac{N_{c}}{2} \operatorname{tr} t^{a} t^{c} t^{b}\right)-6 \cdot\left(C_{\mathrm{F}}-\frac{1}{2}\right) \operatorname{tr} t^{a} t^{c} t^{b}-6 \cdot\left(C_{\mathrm{F}}-\frac{1}{2}\right) \operatorname{tr} t^{a} t^{b} t^{c} \\
& +6 \cdot\left(\left(C_{\mathrm{F}}-\frac{N_{c}}{2}\right) \operatorname{tr} t^{a} t^{c} t^{b}+\left(C_{\mathrm{F}}-1\right) \operatorname{tr} t^{a} t^{b} t^{c}\right)=0 .
\end{aligned}
$$

We then consider the structure

$$
\vec{I} \cdot \frac{z_{2} \vec{p}_{2}-\left(1-z_{2}\right) \vec{k}_{g}}{\left(z_{2} \vec{p}_{2}-\left(1-z_{2}\right) \vec{k}_{g}\right)^{2}} .
$$

For the terms shown in Eqs. (63), (88), (89), (90) the structure above is multiplied with the three-quark wave function

$$
\Psi_{q q q}^{*}\left(x_{1}-x_{g}, \vec{k}_{1}+x_{1} \vec{q}_{12}-\vec{k}_{g}+x_{g} \vec{K}-\vec{q}_{12} ; x_{2}+x_{g}, \vec{k}_{2}+x_{2} \vec{q}_{12}+\vec{k}_{g}-x_{g} \vec{K} ; x_{3}, \vec{k}_{3}+x_{3} \vec{q}_{12}\right),
$$

and the sum of the color factors: 


$$
+6 \cdot \frac{N_{c}}{2} \operatorname{tr} t^{a} t^{b} t^{c}-6 \cdot C_{\mathrm{F}} \operatorname{tr} t^{a} t^{b} t^{c}-6 \cdot\left(C_{\mathrm{F}}-\frac{1}{2}\right) \operatorname{tr} t^{a} t^{b} t^{c}+6 \cdot\left(C_{\mathrm{F}}-\frac{1}{2}-\frac{1}{2 N_{c}}\right) \operatorname{tr} t^{a} t^{b} t^{c}=0
$$

For the terms (75), (94), (95), (96) or (78), (106), (107), (108) the structure in (C27) is multiplied with the three-quark wave function

$$
\Psi_{q q q}^{*}\left(x_{1}-x_{g}, \vec{k}_{1}+x_{1} \vec{q}_{12}-\vec{k}_{g}+x_{g} \vec{K}-\vec{q}_{1} ; x_{2}+x_{g}, \vec{k}_{2}+x_{2} \vec{q}_{12}+\vec{k}_{g}-x_{g} \vec{K} ; x_{3}, \vec{k}_{3}+x_{3} \vec{q}_{12}-\vec{q}_{2}\right)
$$

or

$$
\Psi_{q q q}^{*}\left(x_{1}-x_{g}, \vec{k}_{1}+x_{1} \vec{q}_{12}-\vec{k}_{g}+x_{g} \vec{K}-\vec{q}_{2} ; x_{2}+x_{g}, \vec{k}_{2}+x_{2} \vec{q}_{12}+\vec{k}_{g}-x_{g} \vec{K} ; x_{3}, \vec{k}_{3}+x_{3} \vec{q}_{12}-\vec{q}_{1}\right),
$$

respectively. For the first set of terms, the sum of the color factors yields

$$
\begin{aligned}
& +6 \cdot\left(-\frac{1}{4}\left(T^{c}\right)_{b a}-N_{c} \operatorname{tr} t^{a} t^{c} t^{b}+\frac{1}{4} \operatorname{tr} T^{b} T^{c}\left(D^{a}-T^{a}\right)\right)+6 \cdot\left(2 C_{\mathrm{F}}-\frac{1}{2}-\frac{N_{c}}{2}\right) \operatorname{tr} t^{a} t^{c} t^{b} \\
& +6 \cdot\left(\left(C_{\mathrm{F}}-\frac{N_{c}}{2}\right) \operatorname{tr} t^{a} t^{b} t^{c}+\left(C_{\mathrm{F}}-1\right) \operatorname{tr} t^{a} t^{c} t^{b}\right)+6 \cdot\left(2 C_{\mathrm{F}}-\frac{1}{2}-\frac{N_{c}}{2}\right) \operatorname{tr} t^{a} t^{b} t^{c}=0 .
\end{aligned}
$$

Similarly, for the second set of terms

$$
\begin{aligned}
& +6 \cdot\left(-\frac{1}{4}\left(T^{c}\right)_{a b}-N_{c} \operatorname{tr} t^{a} t^{b} t^{c}+\frac{1}{4} \operatorname{tr} T^{a} T^{c}\left(D^{b}-T^{b}\right)\right)+6 \cdot\left(2 C_{\mathrm{F}}-\frac{1}{2}-\frac{N_{c}}{2}\right) \operatorname{tr} t^{a} t^{b} t^{c} \\
& +6 \cdot\left(\left(C_{\mathrm{F}}-\frac{N_{c}}{2}\right) \operatorname{tr} t^{a} t^{c} t^{b}+\left(C_{\mathrm{F}}-1\right) \operatorname{tr} t^{a} t^{b} t^{c}\right)+6 \cdot\left(2 C_{\mathrm{F}}-\frac{1}{2}-\frac{N_{c}}{2}\right) \operatorname{tr} t^{a} t^{c} t^{b}=0
\end{aligned}
$$

Finally, we consider the set of terms (112), (113), (114) that contain the structure in (C27) multiplied with the three-quark wave function

$$
\Psi_{q q q}^{*}\left(x_{1}-x_{g}, \vec{k}_{1}+x_{1} \vec{q}_{12}-\vec{k}_{g}+x_{g} \vec{K}-\vec{q}_{12} ; x_{2}+x_{g}, \vec{k}_{2}+x_{2} \vec{q}_{12}+\vec{k}_{g}-x_{g} \vec{K} ; x_{3}, \vec{k}_{3}-\left(1-x_{3}\right) \vec{q}_{12}\right) .
$$

For these terms, the sum of the color factors:

$$
+6 \cdot\left(2 C_{\mathrm{F}}-\frac{1}{2}-\frac{N_{c}}{2}\right) \operatorname{tr} t^{a} t^{b} t^{c}+6 \cdot\left(2 C_{\mathrm{F}}-\frac{1}{2}-\frac{N_{c}}{2}\right) \operatorname{tr} t^{a} t^{b} t^{c}+6 \cdot\left(2 C_{\mathrm{F}}-N_{c} C_{\mathrm{F}}\right) \operatorname{tr} t^{a} t^{b} t^{c}=0
$$

We are then left with two more contributions that contain the operator $\vec{I}$. These two contributions involve the structure

$$
\begin{aligned}
\vec{I} & \cdot \frac{z_{2}\left(\vec{p}_{2}-\vec{q}_{1}\right)-\left(1-z_{2}\right) \vec{k}_{g}}{\left(z_{2}\left(\vec{p}_{2}-\vec{q}_{1}\right)-\left(1-z_{2}\right) \vec{k}_{g}\right)^{2}} \\
& \times \Psi_{q q q}^{*}\left(x_{1}-x_{g}, \vec{k}_{1}+x_{1} \vec{q}_{12}-\vec{k}_{g}+x_{g} \vec{K} ; x_{2}+x_{g}, \vec{k}_{2}+x_{2} \vec{q}_{12}-\vec{q}_{1}+\vec{k}_{g}-x_{g} \vec{K} ; x_{3}, \vec{k}_{3}+x_{3} \vec{q}_{12}-\vec{q}_{2}\right)
\end{aligned}
$$

and

$$
\begin{aligned}
\vec{I} & \cdot \frac{z_{2}\left(\vec{p}_{2}-\vec{q}_{2}\right)-\left(1-z_{2}\right) \vec{k}_{g}}{\left(z_{2}\left(\vec{p}_{2}-\vec{q}_{2}\right)-\left(1-z_{2}\right) \vec{k}_{g}\right)^{2}} \\
& \times \Psi_{q q q}^{*}\left(x_{1}-x_{g}, \vec{k}_{1}+x_{1} \vec{q}_{12}-\vec{k}_{g}+x_{g} \vec{K} ; x_{2}+x_{g}, \vec{k}_{2}+x_{2} \vec{q}_{12}-\vec{q}_{2}+\vec{k}_{g}-x_{g} \vec{K} ; x_{3}, \vec{k}_{3}+x_{3} \vec{q}_{12}-\vec{q}_{1}\right)
\end{aligned}
$$

Terms that involve the structure (C36) are given by Eqs. (81), (103), (104), (105), and the sum of the color factors: 


$$
\begin{aligned}
& +6 \cdot\left(-\frac{1}{4}\left(T^{c}\right)_{a b}-\frac{N_{c}}{4}\left(D^{c}\right)_{a b}+\frac{1}{4} \operatorname{tr} T^{c}\left(D^{a}-T^{a}\right) T^{b}\right)+6 \cdot\left(\left(C_{\mathrm{F}}-\frac{N_{c}}{2}\right) \operatorname{tr} t^{a} t^{c} t^{b}+\left(C_{\mathrm{F}}-1\right) \operatorname{tr} t^{a} t^{b} t^{c}\right) \\
& +6 \cdot\left(2 C_{\mathrm{F}}-\frac{1}{2}-\frac{N_{c}}{2}\right) \operatorname{tr} t^{a} t^{c} t^{b}+6 \cdot\left(2 C_{\mathrm{F}}-\frac{1}{2}-\frac{N_{c}}{2}\right) \operatorname{tr} t^{a} t^{b} t^{c}=0 .
\end{aligned}
$$

Terms that involve the structure (C37) are given by Eqs. (84), (109), (110), (111), and the sum of the color factors:

$$
\begin{aligned}
& +6 \cdot\left(+\frac{1}{4}\left(T^{c}\right)_{a b}-\frac{N_{c}}{4}\left(D^{c}\right)_{a b}+\frac{1}{4} \operatorname{tr} T^{c}\left(D^{b}-T^{b}\right) T^{a}\right)+6 \cdot\left(\left(C_{\mathrm{F}}-\frac{N_{c}}{2}\right) \operatorname{tr} t^{a} t^{b} t^{c}+\left(C_{\mathrm{F}}-1\right) \operatorname{tr} t^{a} t^{c} t^{b}\right) \\
& +6 \cdot\left(2 C_{\mathrm{F}}-\frac{1}{2}-\frac{N_{c}}{2}\right) \operatorname{tr} t^{a} t^{b} t^{c}+6 \cdot\left(2 C_{\mathrm{F}}-\frac{1}{2}-\frac{N_{c}}{2}\right) \operatorname{tr} t^{a} t^{c} t^{b}=0 .
\end{aligned}
$$

We then move on and consider contributions which involve the integral operator $J$. Let us start by considering terms which have the structure

$$
\begin{aligned}
& J \frac{z_{2} \vec{p}_{2}-\left(1-z_{2}\right) \vec{k}_{g}}{\left(z_{2} \vec{p}_{2}-\left(1-z_{2}\right) \vec{k}_{g}\right)^{2}} \cdot\left(\frac{z_{2} \vec{p}_{1}-\vec{k}_{g}}{\left(z_{2} \vec{p}_{1}-\vec{k}_{g}\right)^{2}}+\frac{z_{1}\left(\vec{p}_{1}-\vec{q}_{12}\right)-\vec{k}_{g}}{\left(z_{1}\left(\vec{p}_{1}-\vec{q}_{12}\right)-\vec{k}_{g}\right)^{2}}\right) \\
& \quad \times \Psi_{q q q}^{*}\left(x_{1}-x_{g}, \vec{k}_{1}-\left(1-x_{1}\right) \vec{q}_{12}-\vec{k}_{g}+x_{g} \vec{K} ; x_{2}+x_{g}, \vec{k}_{2}+x_{2} \vec{q}_{12}+\vec{k}_{g}-x_{g} \vec{K} ; x_{3}, \vec{k}_{3}+x_{3} \vec{q}_{12}\right) .
\end{aligned}
$$

These terms are given by Eqs. (116), (117), (118), and the sum of the color factors:

$$
+6 \cdot C_{\mathrm{F}} \operatorname{tr} t^{a} t^{b} t^{c}+6 \cdot\left(C_{\mathrm{F}}-\frac{N_{c}+1}{2}\right) \operatorname{tr} t^{a} t^{b} t^{c}-6 \cdot\left(2 C_{\mathrm{F}}-\frac{N_{c}+1}{2}\right) \operatorname{tr} t^{a} t^{b} t^{c}=0 .
$$

We continue by considering the contributions which involve the structure

$$
\begin{aligned}
& J\left(\frac{z_{2} \vec{p}_{2}-\left(1-z_{2}\right) \vec{k}_{g}}{\left(z_{2} \vec{p}_{2}-\left(1-z_{2}\right) \vec{k}_{g}\right)^{2}} \cdot \frac{z_{2} \vec{p}_{1}-\vec{k}_{g}}{\left(z_{2} \vec{p}_{1}-\vec{k}_{g}\right)^{2}}+\frac{z_{2}\left(\vec{p}_{2}-\vec{q}_{2}\right)-\left(1-z_{2}\right) \vec{k}_{g}}{\left(z_{2}\left(\vec{p}_{2}-\vec{q}_{2}\right)-\left(1-z_{2}\right) \vec{k}_{g}\right)^{2}} \cdot \frac{z_{1}\left(\vec{p}_{1}-\vec{q}_{1}\right)-\vec{k}_{g}}{\left(z_{1}\left(\vec{p}_{1}-\vec{q}_{1}\right)-\vec{k}_{g}\right)^{2}}\right) \\
& \quad \times \Psi_{q q q}^{*}\left(x_{1}-x_{g}, \vec{k}_{1}+x_{1} \vec{q}_{12}-\vec{q}_{1}-\vec{k}_{g}+x_{g} \vec{K} ; x_{2}+x_{g}, \vec{k}_{2}+x_{2} \vec{q}_{12}-\vec{q}_{2}+\vec{k}_{g}-x_{g} \vec{K} ; x_{3}, \vec{k}_{3}+x_{3} \vec{q}_{12}\right) .
\end{aligned}
$$

Terms that have this structure are given by Eqs. (119), (120), (121), and the sum of the color factors:

$$
+6 \cdot\left(C_{\mathrm{F}}-\frac{N_{c}+1}{2}\right) \operatorname{tr} t^{a} t^{c} t^{b}+6 \cdot\left(C_{\mathrm{F}}-\frac{N_{c}+1}{2}\right) \operatorname{tr} t^{a} t^{b} t^{c}-6 \cdot\left(C_{\mathrm{F}}-\frac{N_{c}+1}{2}\right) \operatorname{tr}\left(t^{a} t^{b} t^{c}+t^{a} t^{c} t^{b}\right)=0 .
$$

The contributions that contain the structure

$$
\begin{aligned}
& J \frac{z_{2} \vec{p}_{2}-\left(1-z_{2}\right) \vec{k}_{g}}{\left(z_{2} \vec{p}_{2}-\left(1-z_{2}\right) \vec{k}_{g}\right)^{2}} \cdot\left(\frac{z_{2} \vec{p}_{1}-\vec{k}_{g}}{\left(z_{2} \vec{p}_{1}-\vec{k}_{g}\right)^{2}}+\frac{z_{1}\left(\vec{p}_{1}-\vec{q}_{1}\right)-\vec{k}_{g}}{\left(z_{1}\left(\vec{p}_{1}-\vec{q}_{1}\right)-\vec{k}_{g}\right)^{2}}\right) \\
& \quad \times \Psi_{q q q}^{*}\left(x_{1}-x_{g}, \vec{k}_{1}-\left(1-x_{1}\right) \vec{q}_{12}-\vec{q}_{1}-\vec{k}_{g}+x_{g} \vec{K} ; x_{2}+x_{g}, \vec{k}_{2}+x_{2} \vec{q}_{12}+\vec{k}_{g}-x_{g} \vec{K} ; x_{3}, \vec{k}_{3}+x_{3} \vec{q}_{12}-\vec{q}_{2}\right)
\end{aligned}
$$

are given by Eqs. (122), (123), (124). The sum of the color factors:

$$
-6 \cdot\left(2 C_{\mathrm{F}}-\frac{N_{c}+1}{2}\right) \operatorname{tr} t^{a} t^{c} t^{b}-6 \cdot\left(C_{\mathrm{F}}-\frac{N_{c}+1}{2}\right) \operatorname{tr}\left(t^{a} t^{b} t^{c}+t^{a} t^{c} t^{b}\right)-6 \cdot\left(2 C_{\mathrm{F}}-\frac{N_{c}+1}{2}\right) \operatorname{tr} t^{a} t^{b} t^{c}=0 .
$$


The contributions that contain the structure

$$
\begin{aligned}
& J\left(\frac{z_{2} \vec{p}_{2}-\left(1-z_{2}\right) \vec{k}_{g}}{\left(z_{2} \vec{p}_{2}-\left(1-z_{2}\right) \vec{k}_{g}\right)^{2}} \cdot \frac{z_{2} \vec{p}_{1}-\vec{k}_{g}}{\left(z_{2} \vec{p}_{1}-\vec{k}_{g}\right)^{2}}+\frac{z_{2}\left(\vec{p}_{2}-\vec{q}_{1}\right)-\left(1-z_{2}\right) \vec{k}_{g}}{\left(z_{2}\left(\vec{p}_{2}-\vec{q}_{1}\right)-\left(1-z_{2}\right) \vec{k}_{g}\right)^{2}} \cdot \frac{z_{1}\left(\vec{p}_{1}-\vec{q}_{2}\right)-\vec{k}_{g}}{\left(z_{1}\left(\vec{p}_{1}-\vec{q}_{2}\right)-\vec{k}_{g}\right)^{2}}\right) \\
& \quad \times \Psi_{q q q}^{*}\left(x_{1}-x_{g}, \vec{k}_{1}+x_{1} \vec{q}_{12}-\vec{q}_{2}-\vec{k}_{g}+x_{g} \vec{K} ; x_{2}+x_{g}, \vec{k}_{2}+x_{2} \vec{q}_{12}-\vec{q}_{1}+\vec{k}_{g}-x_{g} \vec{K} ; x_{3}, \vec{k}_{3}+x_{3} \vec{q}_{12}\right)
\end{aligned}
$$

are given by Eqs. (125), (126), (127). The sum of the color factors:

$$
+6 \cdot\left(C_{\mathrm{F}}-\frac{N_{c}+1}{2}\right) \operatorname{tr} t^{a} t^{b} t^{c}+6 \cdot\left(C_{\mathrm{F}}-\frac{N_{c}+1}{2}\right) \operatorname{tr} t^{a} t^{c} t^{b}-6 \cdot\left(C_{\mathrm{F}}-\frac{N_{c}+1}{2}\right) \operatorname{tr}\left(t^{a} t^{b} t^{c}+t^{a} t^{c} t^{b}\right)=0 .
$$

Furthermore, the contributions that contain the structure

$$
\begin{aligned}
J & \cdot \frac{z_{2} \vec{p}_{1}-\vec{k}_{g}}{\left(z_{2} \vec{p}_{1}-\vec{k}_{g}\right)^{2}}\left(\frac{z_{2} \vec{p}_{2}-\left(1-z_{2}\right) \vec{k}_{g}}{\left(z_{2} \vec{p}_{2}-\left(1-z_{2}\right) \vec{k}_{g}\right)^{2}}+\frac{z_{2}\left(\vec{p}_{2}-\vec{q}_{12}\right)-\left(1-z_{2}\right) \vec{k}_{g}}{\left(z_{2}\left(\vec{p}_{2}-\vec{q}_{12}\right)-\left(1-z_{2}\right) \vec{k}_{g}\right)^{2}}\right) \\
& \times \Psi_{q q q}^{*}\left(x_{1}-x_{g}, \vec{k}_{1}+x_{1} \vec{q}_{12}-\vec{k}_{g}+x_{g} \vec{K} ; x_{2}+x_{g}, \vec{k}_{2}-\left(1-x_{2}\right) \vec{q}_{12}+\vec{k}_{g}-x_{g} \vec{K} ; x_{3}, \vec{k}_{3}+x_{3} \vec{q}_{12}\right)
\end{aligned}
$$

and

$$
\begin{aligned}
J & \cdot \frac{z_{2} \vec{p}_{1}-\vec{k}_{g}}{\left(z_{2} \vec{p}_{1}-\vec{k}_{g}\right)^{2}}\left(\frac{z_{2} \vec{p}_{2}-\left(1-z_{2}\right) \vec{k}_{g}}{\left(z_{2} \vec{p}_{2}-\left(1-z_{2}\right) \vec{k}_{g}\right)^{2}}+\frac{z_{2}\left(\vec{p}_{2}-\vec{q}_{1}\right)-\left(1-z_{2}\right) \vec{k}_{g}}{\left(z_{2}\left(\vec{p}_{2}-\vec{q}_{1}\right)-\left(1-z_{2}\right) \vec{k}_{g}\right)^{2}}\right) \\
& \times \Psi_{q q q}^{*}\left(x_{1}-x_{g}, \vec{k}_{1}+x_{1} \vec{q}_{12}-\vec{k}_{g}+x_{g} \vec{K} ; x_{2}+x_{g}, \vec{k}_{2}+x_{2} \vec{q}_{12}-\vec{q}_{1}+\vec{k}_{g}-x_{g} \vec{K} ; x_{3}, \vec{k}_{3}+x_{3} \vec{q}_{12}-\vec{q}_{2}\right)
\end{aligned}
$$

are given by Eqs. (128), (129), (130) and Eqs. (131), (132), (133), respectively. Correspondingly, the sum of the color factors

$$
+6 \cdot\left(C_{\mathrm{F}}-\frac{N_{c}+1}{2}\right) \operatorname{tr} t^{a} t^{b} t^{c}+6 \cdot C_{\mathrm{F}} \operatorname{tr} t^{a} t^{b} t^{c}-6 \cdot\left(2 C_{\mathrm{F}}-\frac{N_{c}+1}{2}\right) \operatorname{tr} t^{a} t^{b} t^{c}=0
$$

and

$$
-6 \cdot\left(C_{\mathrm{F}}-\frac{N_{c}+1}{2}\right) \operatorname{tr}\left(t^{a} t^{c} t^{b}+\operatorname{tr} t^{a} t^{b} t^{c}\right)-6 \cdot\left(2 C_{\mathrm{F}}-\frac{N_{c}+1}{2}\right) \operatorname{tr} t^{a} t^{c} t^{b}-6 \cdot\left(2 C_{\mathrm{F}}-\frac{N_{c}+1}{2}\right) \operatorname{tr} t^{a} t^{b} t^{c}=0 .
$$

The final set of contributions are given by Eqs. (134), (135), (136); Eqs. (137), (138), (139); and Eqs. (140), (141), (142). These terms contain three independent structures

$$
\begin{aligned}
& J \frac{z_{2} \vec{p}_{2}-\left(1-z_{2}\right) \vec{k}_{g}}{\left(z_{2} \vec{p}_{2}-\left(1-z_{2}\right) \vec{k}_{g}\right)^{2}} \cdot\left(\frac{z_{2} \vec{p}_{1}-\vec{k}_{g}}{\left(z_{2} \vec{p}_{1}-\vec{k}_{g}\right)^{2}}+\frac{z_{2}\left(\vec{p}_{2}-\vec{q}_{2}\right)-\left(1-z_{2}\right) \vec{k}_{g}}{\left(z_{2}\left(\vec{p}_{2}-\vec{q}_{2}\right)-\left(1-z_{2}\right) \vec{k}_{g}\right)^{2}}\right) \\
& \quad \times \Psi_{q q q}^{*}\left(x_{1}-x_{g}, \vec{k}_{1}+x_{1} \vec{q}_{12}-\vec{q}_{2}-\vec{k}_{g}+x_{g} \vec{K} ; x_{2}+x_{g}, \vec{k}_{2}+x_{2} \vec{q}_{12}+\vec{k}_{g}-x_{g} \vec{K} ; x_{3}, \vec{k}_{3}+x_{3} \vec{q}_{12}-\vec{q}_{1}\right), \\
& J \frac{z_{2} \vec{p}_{1}-\vec{k}_{g}}{\left(z_{2} \vec{p}_{1}-\vec{k}_{g}\right)^{2}} \cdot\left(\frac{z_{2} \vec{p}_{2}-\left(1-z_{2}\right) \vec{k}_{g}}{\left(z_{2} \vec{p}_{2}-\left(1-z_{2}\right) \vec{k}_{g}\right)^{2}}+\frac{z_{2}\left(\vec{p}_{2}-\vec{q}_{2}\right)-\left(1-z_{2}\right) \vec{k}_{g}}{\left(z_{2}\left(\vec{p}_{2}-\vec{q}_{2}\right)-\left(1-z_{2}\right) \vec{k}_{g}\right)^{2}}\right) \\
& \quad \times \Psi_{q q q}^{*}\left(x_{1}-x_{g}, \vec{k}_{1}+x_{1} \vec{q}_{12}-\vec{k}_{g}+x_{g} \vec{K} ; x_{2}+x_{g}, \vec{k}_{2}+x_{2} \vec{q}_{12}-\vec{q}_{2}+\vec{k}_{g}-x_{g} \vec{K} ; x_{3}, \vec{k}_{3}+x_{3} \vec{q}_{12}-\vec{q}_{1}\right),
\end{aligned}
$$

and

$$
\begin{aligned}
& J \frac{z_{2} \vec{p}_{1}-\vec{k}_{g}}{\left(z_{2} \vec{p}_{1}-\vec{k}_{g}\right)^{2}} \cdot \frac{z_{2} \vec{p}_{2}-\left(1-z_{2}\right) \vec{k}_{g}}{\left(z_{2} \vec{p}_{2}-\left(1-z_{2}\right) \vec{k}_{g}\right)^{2}} \\
& \quad \times \Psi_{q q q}^{*}\left(x_{1}-x_{g}, \vec{k}_{1}+x_{1} \vec{q}_{12}-\vec{k}_{g}+x_{g} \vec{K} ; x_{2}+x_{g}, \vec{k}_{2}+x_{2} \vec{q}_{12}+\vec{k}_{g}-x_{g} \vec{K} ; x_{3}, \vec{k}_{3}-\left(1-x_{3}\right) \vec{q}_{12}\right),
\end{aligned}
$$


respectively. Correspondingly, the sum of the color factors

$$
\begin{aligned}
& -6 \cdot\left(2 C_{\mathrm{F}}-\frac{N_{c}+1}{2}\right) \operatorname{tr} t^{a} t^{b} t^{c}-6 \cdot\left(C_{\mathrm{F}}-\frac{N_{c}+1}{2}\right) \operatorname{tr}\left(t^{a} t^{b} t^{c}+t^{a} t^{c} t^{b}\right)-6 \cdot\left(2 C_{\mathrm{F}}-\frac{N_{c}+1}{2}\right) \operatorname{tr} t^{a} t^{c} t^{b}=0 \\
& -6 \cdot\left(C_{\mathrm{F}}-\frac{N_{c}+1}{2}\right) \operatorname{tr}\left(t^{a} t^{b} t^{c}+t^{a} t^{c} t^{b}\right)-6 \cdot\left(2 C_{\mathrm{F}}-\frac{N_{c}+1}{2}\right) \operatorname{tr} t^{a} t^{b} t^{c}-6 \cdot\left(2 C_{\mathrm{F}}-\frac{N_{c}+1}{2}\right) \operatorname{tr} t^{a} t^{c} t^{b}=0
\end{aligned}
$$

and

$$
-6 \cdot 2\left(2 C_{\mathrm{F}}-\frac{N_{c}+1}{2}\right) \operatorname{tr} t^{a} t^{b} t^{c}-6 \cdot 2\left(2 C_{\mathrm{F}}-\frac{N_{c}+1}{2}\right) \operatorname{tr} t^{a} t^{b} t^{c}-6 \cdot 2 C_{\mathrm{F}}\left(2-N_{c}\right) \operatorname{tr} t^{a} t^{b} t^{c}=0 .
$$

This concludes our check that the sum of all UV finite diagrams cancel when either $\vec{q}_{1}$ or $\vec{q}_{3} \rightarrow 0$.

[1] A. Dumitru and R. Paatelainen, Sub-femtometer scale color charge fluctuations in a proton made of three quarks and a gluon, Phys. Rev. D 103, 034026 (2021).

[2] A. Dumitru, H. Mäntysaari, and R. Paatelainen, Color charge correlations in the proton at NLO: Beyond geometry based intuition, Phys. Lett. B 820, 136560 (2021).

[3] A. Dumitru, H. Mäntysaari, R. Paatelainen, K. Roy, F. Salazar, and B. Schenke, Azimuthal correlations in diffractive scattering at the Electron-Ion Collider, arXiv:2105.10144.

[4] A. H. Mueller in Cargese 2001, QCD Perspectives on Hot and Dense Matter (Springer, Dordrecht, 2001), pp. 45-72.

[5] Y. V. Kovchegov and E. Levin, Quantum Chromodynamics at High Energy (Cambridge University Press, Cambridge, England, 2012), Vol. 33.

[6] J. Bartels, High-energy behavior in a nonabelian gauge theory (II): First corrections to $T_{n \rightarrow m}$ beyond the leading $\ln s$ approximation, Nucl. Phys. B175, 365 (1980).

[7] T. Jaroszewicz, Infrared divergences and Regge behavior in QCD, Acta Phys. Pol. B 11, 965 (1980).

[8] J. Kwiecinski and M. Praszalowicz, Three gluon integral equation and odd $\mathrm{C}$ singlet Regge singularities in QCD, Phys. Lett. 94B, 413 (1980).

[9] M. A. Braun, Odderon and QCD, arXiv:hep-ph/9805394.

[10] C. Ewerz, The odderon in quantum chromodynamics, arXiv:hep-ph/0306137.

[11] A. Kovner and M. Lublinsky, Odderon and seven Pomerons: QCD Reggeon field theory from JIMWLK evolution, J. High Energy Phys. 02 (2007) 058.

[12] V. M. Abazov et al. (D0, TOTEM Collaboration), Comparison of $p p$ and $p \bar{p}$ Differential Elastic Cross Sections and Observation of the Exchange of a Colorless C-Odd Gluonic Compound, Phys. Rev. Lett. 127, 062003 (2021).

[13] G. Antchev et al. (TOTEM Collaboration), First determination of the $\rho$ parameter at $\sqrt{s}=13 \mathrm{TeV}$ : Probing the existence of a colourless C-odd three-gluon compound state, Eur. Phys. J. C 79, 785 (2019).
[14] E. Martynov and B. Nicolescu, Odderon effects in the differential cross-sections at Tevatron and LHC energies, Eur. Phys. J. C 79, 461 (2019).

[15] I. Balitsky, Operator expansion for high-energy scattering, Nucl. Phys. B463, 99 (1996).

[16] I. Balitsky, Factorization and high-energy effective action, Phys. Rev. D 60, 014020 (1999).

[17] I. Balitsky, Effective field theory for the small-x evolution, Phys. Lett. B 518, 235 (2001).

[18] J. Jalilian-Marian, A. Kovner, A. Leonidov, and H. Weigert, The BFKL equation from the Wilson renormalization group, Nucl. Phys. B504, 415 (1997).

[19] J. Jalilian-Marian, A. Kovner, A. Leonidov, and H. Weigert, The Wilson renormalization group for low $\mathrm{x}$ physics: Towards the high density regime, Phys. Rev. D 59, 014014 (1998).

[20] J. Jalilian-Marian, A. Kovner, and H. Weigert, The Wilson renormalization group for low x physics: Gluon evolution at finite parton density, Phys. Rev. D 59, 014015 (1998).

[21] E. Iancu, A. Leonidov, and L. D. McLerran, The renormalization group equation for the color glass condensate, Phys. Lett. B 510, 133 (2001).

[22] E. Iancu, A. Leonidov, and L.D. McLerran, Nonlinear gluon evolution in the color glass condensate. I, Nucl. Phys. A692, 583 (2001).

[23] E. Ferreiro, E. Iancu, A. Leonidov, and L. McLerran, Nonlinear gluon evolution in the color glass condensate. II, Nucl. Phys. A703, 489 (2002).

[24] H. Weigert, Unitarity at small Bjorken x, Nucl. Phys. A703, 823 (2002).

[25] Y. V. Kovchegov, Small-x $F_{2}$ structure function of a nucleus including multiple pomeron exchanges, Phys. Rev. D 60, 034008 (1999).

[26] Y. V. Kovchegov, L. Szymanowski, and S. Wallon, Perturbative odderon in the dipole model, Phys. Lett. B 586, 267 (2004).

[27] Y. Hatta, E. Iancu, K. Itakura, and L. McLerran, Odderon in the color glass condensate, Nucl. Phys. A760, 172 (2005). 
[28] T. Lappi, A. Ramnath, K. Rummukainen, and H. Weigert, JIMWLK evolution of the odderon, Phys. Rev. D 94, 054014 (2016).

[29] J. Zhou, Transverse single spin asymmetries at small $\mathrm{x}$ and the anomalous magnetic moment, Phys. Rev. D 89, 074050 (2014).

[30] D. Boer, M. G. Echevarria, P. Mulders, and J. Zhou, Single Spin Asymmetries from a Single Wilson Loop, Phys. Rev. Lett. 116, 122001 (2016).

[31] X. Yao, Y. Hagiwara, and Y. Hatta, Computing the gluon Sivers function at small- $x$, Phys. Lett. B 790, 361 (2019).

[32] P. Hägler, B. Pire, L. Szymanowski, and O. Teryaev, Hunting the QCD-Odderon in hard diffractive electroproduction of two pions, Phys. Lett. B 535, 117 (2002); Phys. Lett. B 540, 324(E) (2002).

[33] P. Hägler, B. Pire, L. Szymanowski, and O. Teryaev, Pomeron-odderon interference effects in electroproduction of two pions, Eur. Phys. J. C 26, 261 (2002).

[34] A. Dumitru and T. Stebel, Multiquark matrix elements in the proton and three gluon exchange for exclusive $\eta_{c}$ production in photon-proton diffractive scattering, Phys. Rev. D 99, 094038 (2019).

[35] J. Czyzewski, J. Kwiecinski, L. Motyka, and M. Sadzikowski, Exclusive $\eta_{c}$ photoproduction and electroproduction at HERA as a possible probe of the odderon singularity in QCD, Phys. Lett. B 398, 400 (1997); Phys. Lett. B 411, 402(E) (1997).

[36] R. Engel, D. Ivanov, R. Kirschner, and L. Szymanowski, Diffractive meson production from virtual photons with odd charge-parity exchange, Eur. Phys. J. C 4, 93 (1998).

[37] W. Kilian and O. Nachtmann, Single pseudoscalar meson production in diffractive e p scattering, Eur. Phys. J. C 5, 317 (1998).

[38] M. Rueter, H. G. Dosch, and O. Nachtmann, Odd CP contributions to diffractive processes, Phys. Rev. D 59, 014018 (1998).

[39] J. L. Albacete and A. Soto-Ontoso, Hot spots and the hollowness of proton-proton interactions at high energies, Phys. Lett. B 770, 149 (2017).

[40] J. L. Albacete, H. Petersen, and A. Soto-Ontoso, Correlated wounded hot spots in proton-proton interactions, Phys. Rev. C 95, 064909 (2017).

[41] J. L. Albacete, H. Petersen, and A. Soto-Ontoso, Symmetric cumulants as a probe of the proton substructure at LHC energies, Phys. Lett. B 778, 128 (2018).

[42] T. Csörgő, R. Pasechnik, and A. Ster, Proton structure and hollowness from Lévy imaging of $p p$ elastic scattering, Eur. Phys. J. C 80, 126 (2020).

[43] H. Mäntysaari, Review of proton and nuclear shape fluctuations at high energy, Rep. Prog. Phys. 83, 082201 (2020).

[44] D. Boer et al., Gluons and the quark sea at high energies: Distributions, polarization, tomography, arXiv:1108.1713.

[45] A. Accardi et al., Electron ion collider: The next QCD frontier: Understanding the glue that binds us all, Eur. Phys. J. A 52, 268 (2016).

[46] E. Aschenauer, S. Fazio, J. Lee, H. Mäntysaari, B. Page, B. Schenke, T. Ullrich, R. Venugopalan, and P. Zurita, The electron-ion collider: Assessing the energy dependence of key measurements, Rep. Prog. Phys. 82, 024301 (2019).
[47] R. Abdul Khalek et al., Science requirements and detector concepts for the electron-ion collider: EIC yellow report, arXiv:2103.05419.

[48] A. Dumitru, G. A. Miller, and R. Venugopalan, Extracting many-body color charge correlators in the proton from exclusive DIS at large Bjorken x, Phys. Rev. D 98, 094004 (2018).

[49] A. Dumitru, V. Skokov, and T. Stebel, Subfemtometer scale color charge correlations in the proton, Phys. Rev. D 101, 054004 (2020).

[50] J. Bartels and L. Motyka, Baryon scattering at high energies: Wave function, impact factor, and gluon radiation, Eur. Phys. J. C 55, 65 (2008).

[51] G. Beuf, Improving the kinematics for low-x QCD evolution equations in coordinate space, Phys. Rev. D 89, 074039 (2014).

[52] B. Ducloué, E. Iancu, A. Mueller, G. Soyez, and D. Triantafyllopoulos, Non-linear evolution in QCD at highenergy beyond leading order, J. High Energy Phys. 04 (2019) 081.

[53] R. Boussarie and Y. Mehtar-Tani, A novel formulation of the unintegrated gluon distribution for DIS, arXiv:2006.14569.

[54] Y. V. Kovchegov and M. D. Sievert, A new mechanism for generating a single transverse spin asymmetry, Phys. Rev. D 86, 034028 (2012); Phys. Rev. D 86, 079906(E) (2012).

[55] F. Schlumpf, Relativistic constituent quark model of electroweak properties of baryons, Phys. Rev. D 47, 4114 (1993); Phys. Rev. D 49, 6246(E) (1994).

[56] S. J. Brodsky and F. Schlumpf, Wave function independent relations between the nucleon axial coupling $\mathrm{g}(\mathrm{A})$ and the nucleon magnetic moments, Phys. Lett. B 329, 111 (1994).

[57] K. J. Golec-Biernat and A. M. Stasto, On solutions of the balitsky-kovchegov equation with impact parameter, Nucl. Phys. B668, 345 (2003).

[58] J. Berger and A. Stasto, Numerical solution of the nonlinear evolution equation at small $\mathrm{x}$ with impact parameter and beyond the 11 approximation, Phys. Rev. D 83, 034015 (2011).

[59] J. Cepila, J. Contreras, and M. Matas, Collinearly improved kernel suppresses Coulomb tails in the impact-parameter dependent Balitsky-Kovchegov evolution, Phys. Rev. D 99, 051502 (2019).

[60] H. Mäntysaari and B. Schenke, Confronting impact parameter dependent JIMWLK evolution with HERA data, Phys. Rev. D 98, 034013 (2018).

[61] D. Bendova, J. Cepila, J. Contreras, and M. Matas, Solution to the Balitsky-Kovchegov equation with the collinearly improved kernel including impact-parameter dependence, Phys. Rev. D 100, 054015 (2019).

[62] D. Binosi, J. Collins, C. Kaufhold, and L. Theussl, JaxoDraw: A graphical user interface for drawing Feynman diagrams. Version 2.0 release notes, Comput. Phys. Commun. 180, 1709 (2009).

[63] G. Lepage and S. J. Brodsky, Exclusive processes in perturbative quantum chromodynamics, Phys. Rev. D 22, 2157 (1980).

[64] S. J. Brodsky, H.-C. Pauli, and S. S. Pinsky, Quantum chromodynamics and other field theories on the light cone, Phys. Rep. 301, 299 (1998). 
[65] S. J. Brodsky, D. S. Hwang, B.-Q. Ma, and I. Schmidt, Light cone representation of the spin and orbital angular momentum of relativistic composite systems, Nucl. Phys. 593B, 311 (2001).

[66] H. Hänninen, T. Lappi, and R. Paatelainen, One-loop corrections to light cone wave functions: The dipole picture DIS cross section, Ann. Phys. (Amsterdam) 393, 358 (2018).

[67] T. Lappi and R. Paatelainen, The one loop gluon emission light cone wave function, Ann. Phys. (Amsterdam) 379, 34 (2017).

[68] V. Gribov and L. Lipatov, Deep inelastic e p scattering in perturbation theory, Sov. J. Nucl. Phys. 15, 438 (1972).
[69] V. Gribov and L. Lipatov, $e^{+} e^{-}$pair annihilation and deep inelastic e p scattering in perturbation theory, Sov. J. Nucl. Phys. 15, 675 (1972).

[70] G. Altarelli and G. Parisi, Asymptotic freedom in parton language, Nucl. Phys. B126, 298 (1977).

[71] Y. L. Dokshitzer, Calculation of the structure functions for deep inelastic scattering and $e^{+} e^{-}$annihilation by perturbation theory in quantum chromodynamics, Sov. Phys. JETP 46, 641 (1977).

[72] J. Bartels and C. Ewerz, Unitarity corrections in high-energy QCD, J. High Energy Phys. 09 (1999) 026.

[73] C. Ewerz, Reggeization in high-energy QCD, J. High Energy Phys. 04 (2001) 031. 